University of Tennessee Health Science Center

UTHSC Digital Commons

$12-2008$

\title{
Studies to Characterize the Requirements for the Binding and Release of ERdj3, a Mammalian ER DnaJ Homolog, from Substrates
}

Yi Jin

University of Tennessee Health Science Center

Follow this and additional works at: https://dc.uthsc.edu/dissertations

Part of the Medical Molecular Biology Commons

\section{Recommended Citation}

Jin, $\mathrm{Yi}$, "Studies to Characterize the Requirements for the Binding and Release of ERdj3, a Mammalian ER DnaJ Homolog, from Substrates" (2008). Theses and Dissertations (ETD). Paper 348. http://dx.doi.org/ 10.21007/etd.cghs.2008.0155.

This Dissertation is brought to you for free and open access by the College of Graduate Health Sciences at UTHSC Digital Commons. It has been accepted for inclusion in Theses and Dissertations (ETD) by an authorized administrator of UTHSC Digital Commons. For more information, please contact jwelch30@uthsc.edu. 


\title{
Studies to Characterize the Requirements for the Binding and Release of ERdj3, a Mammalian ER DnaJ Homolog, from Substrates
}

\begin{abstract}
Heat shock protein 70s (Hsp70s) and their DnaJ co-factors exist in all organisms and in all eukaryotic organelles. These highly conserved chaperone pairs facilitate a large number of cellular processes. ERdj3 was identified as a soluble, lumenal DnaJ family member that binds to unassembled immunoglobulin heavy chains $(\mathrm{HC})$ along with the BiP chaperone complex in the endoplasmic reticulum of mammalian cells. Here we demonstrate that ERdj3 binds directly to two unfolded substrates: immunoglobulin $\mathrm{YHC}$ and denatured firefly luciferase. Using mutagenesis studies on ERdj3 in both in vivo and in vitro binding assays, we defined ERdj3's critical amino acids in domain I that contribute to substrate binding and demonstrated that ERdj3 forms dimers, which are important for substrate binding. We suggested that these features are conserved among all type I and type II DnaJ proteins. Somewhat unexpectedly, we found that domain II, which is highly conserved among ERdj3 homologues but very different from domain II of Ydj1, was also essential for substrate binding. Recent studies have demonstrated that Hsp70s can interact in vitro with some but not all DnaJ proteins from different organelles or even different organisms. To better understand restrictions on Hsp70/DnaJ interactions, we expressed ERdj3 in both the yeast ER and cytosol. Our data revealed that the ability to complement loss of the resident DnaJ proteins in either of these organelles was most dependent on its ability to interact with the resident Hsp70. Mutations in ERdj3 that affected substrate binding were unable to complement loss of Ydj1, arguing that substrate binding ability is also important. Finally, previous studies suggested that DnaJ proteins often bind to unfolded substrates initially and recruit their Hsp70 partners. DnaJ proteins stimulate the ATPase activity of its Hsp70 partner and induce a conformational change in the Hsp70 to stabilize its binding to substrate. By some unknown mechanism the DnaJ protein is then released. We established an in vitro model to examine the requirements for the release of ERdj3 from substrates and found that BiP promoted the release of ERdj3 only in the presence of ATP. Mutations in ERdj3 or BiP that disrupted their interaction interrupted the release of ERdj3. BiP mutants that cannot bind to ATP or undergo a nucleotide-induced conformational change were also unable to release ERdj3. These results demonstrate that a functional interaction between ERdj3 and BiP, including both a direct interaction and the ability to stimulate BiP's ATPase activity are required to release ERdj3 from substrate. Furthermore they suggest that the interaction with BiP may induce a reciprocal change in ERdj3 that triggers its release from substrates. Based on similarities among DnaJs and Hsp70s, this is likely to be applicable to other Hsp70/DnaJ pairs.
\end{abstract}

\section{Document Type}

Dissertation

\section{Degree Name}

Doctor of Philosophy (PhD)

\section{Program}

Molecular Sciences

Research Advisor

Linda M. Hendershot, Ph.D.

Keywords

BiP, DnaJ-like proteins, Endoplasmic reticulum, Protein folding 


\section{Subject Categories}

Medical Molecular Biology | Medical Sciences | Medicine and Health Sciences 


\title{
STUDIES TO CHARACTERIZE THE REQUIREMENTS FOR THE BINDING AND RELEASE OF ERDJ3, A MAMMALIAN ER DNAJ HOMOLOG, FROM SUBSTRATES
}

\author{
A Dissertation \\ Presented for \\ The Graduate Studies Council \\ The University of Tennessee \\ Health Science Center
}

\author{
In Partial Fulfillment \\ Of the Requirements for the Degree \\ Doctor of Philosophy \\ From The University of Tennessee
}

By

Yi Jin

December, 2008 
Copyright (C) 2008 by Yi Jin

All rights reserved 


\section{ACKNOWLEDGEMENTS}

I would like to express my greatest appreciation and gratitude to my advisor, Dr. Linda Hendershot for her encouragement, guidance and unconditional support. With great patience, Dr. Hendershot led me through the journey to become a rigorous researcher. She instills me with her possession of knowledge and her enthusiasm in science.

I thank my committee members, Dr. DarioVignali, Dr. John Cox, Dr. Michael Whitt and Dr. Susan Senogles, for their generous support, guidance and encouragement. Special thanks go to Dr. Patrick Ryan, PhD director.

I want to thank everyone in Dr. Linda Hendershot's Lab, Dr. Ying Shen, Dr. Yuichiro Shimizo, Dr.Yuki Shimizo, Ethel Pereira, Melissa Mann, Walid Awad and all previous members for their helpful scientific discussion, technical support, and friendship.

I also would like to thank Dr. Jeffrey L. Brodsky and Shruthi S. Vembar from University of Pittsburgh for their contribution to yeast related studies.

I am grateful to have met many wonderful friends during my study at the University of Tennessee Health Science Center, and St. Jude Children's research hospital.

I am eternally thankful for my parents and my husband. Without their unconditional love and support, this work would not have been possible.

Finally, I would like to acknowledge that part of my dissertation work, namely Chapter 4, modified from an article accepted by EMBO Journal (Jin Y, Awad W, Petrova $\mathrm{K}$, and Hendershot LM, Regulated release of ERdj3 from unfolded proteins by BiP. $E M B O J$. In press.). 


\begin{abstract}
Heat shock protein 70s (Hsp70s) and their DnaJ co-factors exist in all organisms and in all eukaryotic organelles. These highly conserved chaperone pairs facilitate a large number of cellular processes. ERdj3 was identified as a soluble, lumenal DnaJ family member that binds to unassembled immunoglobulin heavy chains (HC) along with the BiP chaperone complex in the endoplasmic reticulum of mammalian cells. Here we demonstrate that ERdj3 binds directly to two unfolded substrates: immunoglobulin $\gamma \mathrm{HC}$ and denatured firefly luciferase. Using mutagenesis studies on ERdj3 in both in vivo and in vitro binding assays, we defined ERdj3's critical amino acids in domain I that contribute to substrate binding and demonstrated that ERdj3 forms dimers, which are important for substrate binding. We suggested that these features are conserved among all type I and type II DnaJ proteins. Somewhat unexpectedly, we found that domain II, which is highly conserved among ERdj3 homologues but very different from domain II of Ydj1, was also essential for substrate binding. Recent studies have demonstrated that Hsp70s can interact in vitro with some but not all DnaJ proteins from different organelles or even different organisms. To better understand restrictions on Hsp70/DnaJ interactions, we expressed ERdj3 in both the yeast ER and cytosol. Our data revealed that the ability to complement loss of the resident DnaJ proteins in either of these organelles was most dependent on its ability to interact with the resident Hsp70. Mutations in ERdj3 that affected substrate binding were unable to complement loss of Ydj1, arguing that substrate binding ability is also important. Finally, previous studies suggested that DnaJ proteins often bind to unfolded substrates initially and recruit their Hsp70 partners. DnaJ proteins stimulate the ATPase activity of its Hsp70 partner and induce a conformational change in the Hsp70 to stabilize its binding to substrate. By some unknown mechanism the DnaJ protein is then released. We established an in vitro model to examine the requirements for the release of ERdj3 from substrates and found that BiP promoted the release of ERdj3 only in the presence of ATP. Mutations in ERdj3 or BiP that disrupted their interaction interrupted the release of ERdj3. BiP mutants that cannot bind to ATP or undergo a nucleotide-induced conformational change were also unable to release ERdj3. These results demonstrate that a functional interaction between ERdj3 and BiP, including both a direct interaction and the ability to stimulate BiP's ATPase activity are required to release ERdj3 from substrate. Furthermore they suggest that the interaction with BiP may induce a reciprocal change in ERdj3 that triggers its release from substrates. Based on similarities among DnaJs and Hsp70s, this is likely to be applicable to other Hsp70/DnaJ pairs.
\end{abstract}




\section{TABLE OF CONTENTS}

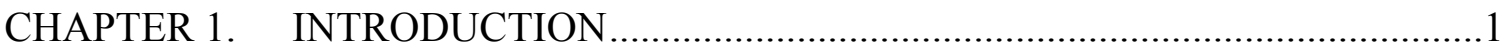

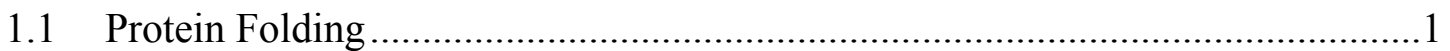

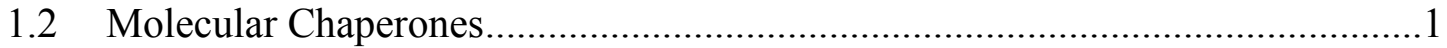

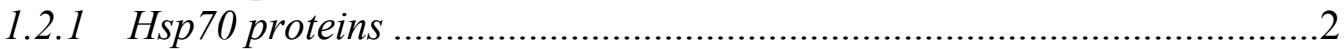

1.2.2 Mammalian ER Hsp70 .....................................................................

1.3 Hsp40/DnaJ Protein................................................................................

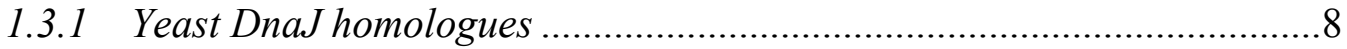

1.3.2 Mammalian ER DnaJ homologues ..................................................

1.4 Nucleotide Exchange Factors ....................................................................

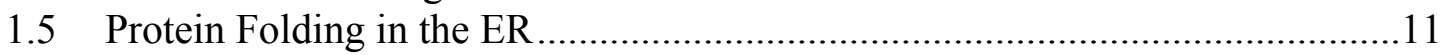

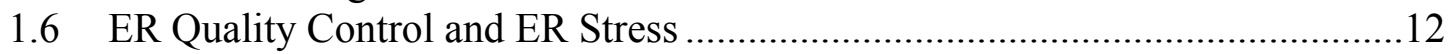

1.7 DnaJ Proteins as Specifiers of Hsp70 Function ...........................................13

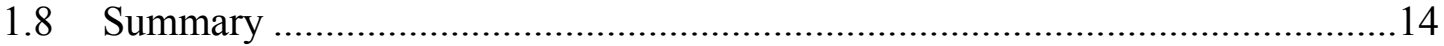

CHAPTER 2. ERDJ3, A LUMENAL ER DNAJ HOMOLOG, BINDS DIRECTLY TO UNFOLODED PROTEINS IN THE MAMMALIAN ER: IDENTIFICATION OF CRITICAL RESIDUES....................15

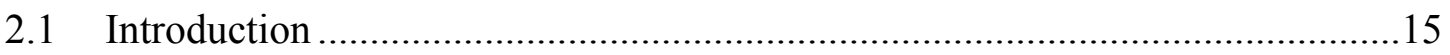

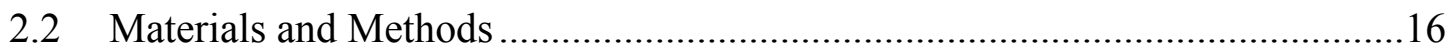

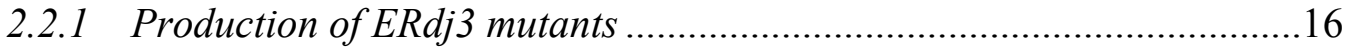

2.2.2 Cell culture, transfection and immunoprecipitation .............................17

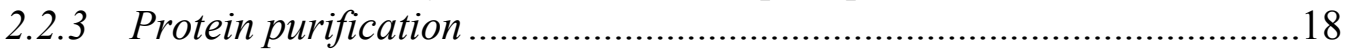

2.2.4 Measurement of complex formation between ERdj3 proteins and denatured luciferase ......................................................................18

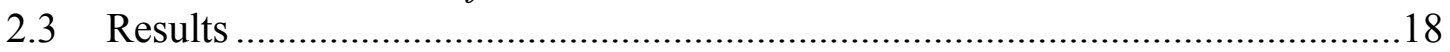

2.3.1 Modeling ERdj3's secondary structure .............................................18

2.3.2 Mapping ERdj3's substrate binding domain in vivo .............................19

2.3.3 Binding of ERdj3 to purified denatured luciferase in vitro....................22

2.3.4 ERdj3 forms dimer, which is important for substrate binding ...............24

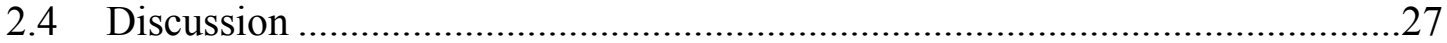

CHAPTER 3. FUNCTIONAL CONSERVATION BETWEEN ERDJ3 AND YDJ1, DNAJ HOMOLOGUES IN THE MAMMALIAN ER AND YEAST

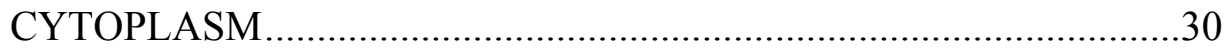

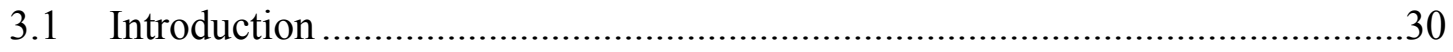

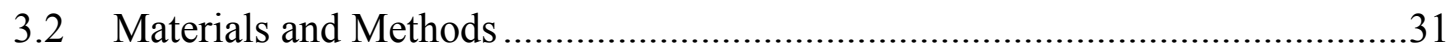

3.2.1 Preparation of Ydj1 and ERdj3 constructs .........................................31

3.2.2 Expression and detection of Ydj1 in mammalian cells..........................32

3.2.3 Protein expression, purification and ATPase assay ...............................32

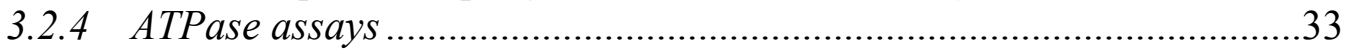


3.2.5 Rescue of the slow growth phenotype of the Ydj1 mutant strain............33

3.3 Results ...

3.3.1 Ydj1 expressed in the mammalian ER binds to BiP and ERdj3 substrates.

3.3.2 Overexpression of ERdj3 does not rescue the temperature-sensitive defect of the $\triangle$ scj1 14jem 1 strain....

3.3.3 Overexpression of cytosolically localized ERdj3 rescues the temperature-sensitive defect of the hlj1D ydj1-151 strain.

3.3.4 Mutations in ERdj3 that affect substrate binding are unable to rescue the slow growth phenotype of hlj1- ydj1-151 strain

3.4 Discussion

CHAPTER 4. REGULATED RELEASE OF ERDJ3 FROM UNFOLDED PROTEINS BY BIP

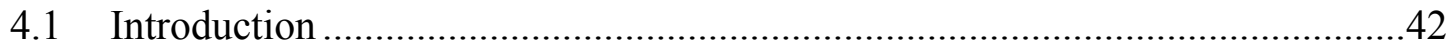

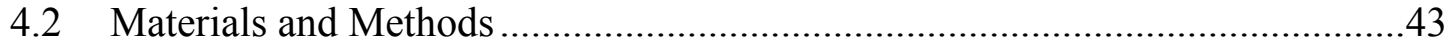

4.2.1 Cell culture, transfection, and immunoprecipitation .............................43

4.2.2 In vitro translation and heavy chain binding assay ..............................44

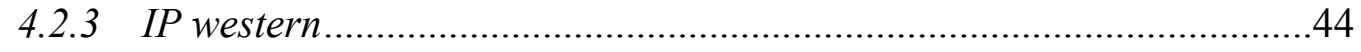

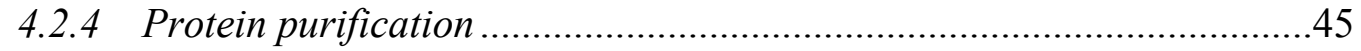

4.2.5 Measurement of complex formation between ERdj3 proteins and denatured luciferase ........................................................................45

4.2.6 Release of ERdj3 from luciferase ......................................................45

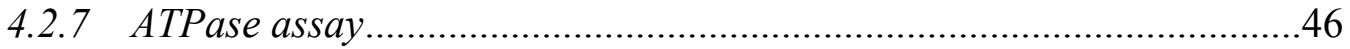

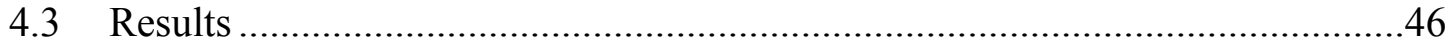

4.3.1 Comparison of the effects of J domain mutations on ERdj3's ability to associate with substrate both in vivo and in vitro ..............................46

4.3.2 Development of an in vitro system to detect binding and release of ERdj 3 from substrates .....................................................................48

4.3.3 ATP does not affect the binding of wild type ERdj3 or the QPD and HPN mutants to substrate in vitro ......................................................50

4.3.4 BiP promoted the release of wild-type ERdj3 from chemically denatured luciferase in the presence of ATP.

4.3.5 BiP mutants that do not interact with ERdj3 failed to promote the release of ERdj3 from substrate ........................................................50

4.3.6 Wild type BiP does not release two ERdj3 mutants, QPD and HPN, from luciferase.

4.4 Discussion

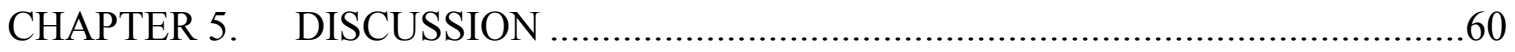

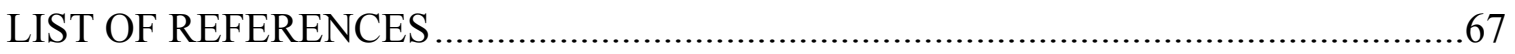

APPENDIX A. SUPPLEMENTAL MATERIAL FOR CHAPTER 3 .........................83 
APPENDIX B. SUPPLEMENTAL MATERIAL FOR CHAPTER 4 .........................87

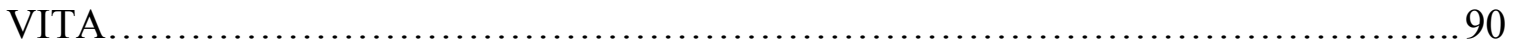




\section{LIST OF FIGURES}

Figure 1-1. Hsp70s' ATPase cycle and substrate binding .......................................... 4

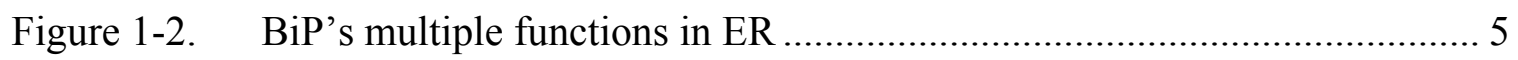

Figure 1-3. Three types of DnaJ family members ………………………………... 7

Figure 1-4. Characterization of mammalian ER DnaJ proteins................................... 10

Figure 2-1. Structural comparisons of the DnaJ proteins ......................................... 20

Figure 2-2. Determination of the ability of wild-type and mutant ERdj3 to bind

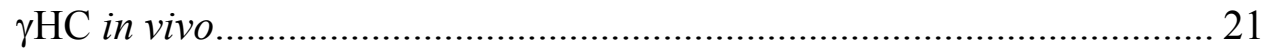

Figure 2-3. Both domain I and domain II of ERdj3 contribute to its ability to bind

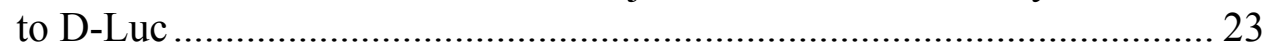

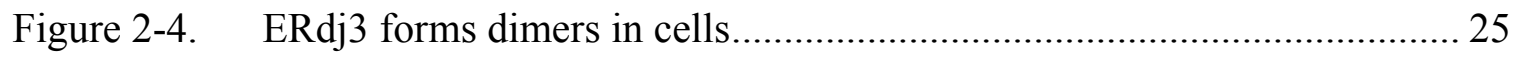

Figure 2-5. Deletion of domain III or mutation of Phe326 to aspartic acid affects

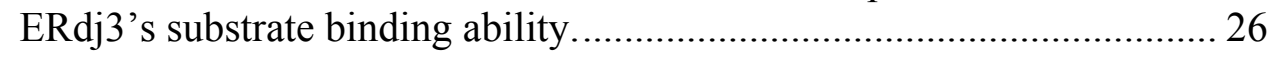

Figure 3-1. Mammalian ER expressed Ydj1 interacts with both BiP and אLC........... 35

Figure 3-2. ER expressed ERdj3 does not rescue the slow growth phenotype of the

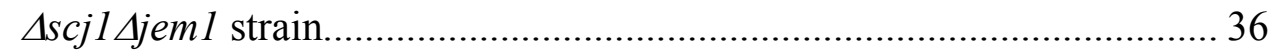

Figure 3-3. Cytosolic expression of ERdj3 rescues the temperature-sensitive

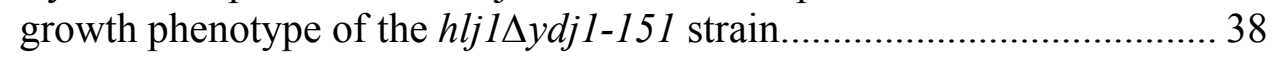

Figure 3-4. ERdj3 substrate binding mutants cannot rescue the temperature sensitive growth phenotype of the hlj1 $\Delta y d j 1-151$ strain

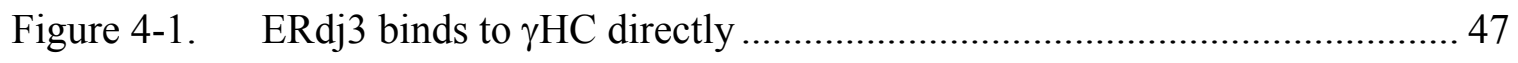

Figure 4-2. WT and QPD ERdj3 bind to denatured luciferase similarly in vitro ........ 49

Figure 4-3. WT and mutant (QPD and HPN) ERdj3 bind to D-Luc similarly and ATP does not affect their binding ......................................................... 51

Figure 4-4. BiP releases ERdj3 from D-Luc in an ATP-dependent manner ............... 52

Figure 4-5. Only WT BiP releases ERdj3 from D-Luc ............................................... 54 
Figure 4-6. Wild-type BiP can only release wild-type ERdj3 from D-Luc......

Figure 4-7. R197H BiP cannot be released from D-Luc by either wild-type ERdj3 or the QPD mutant ....................................................................... 57

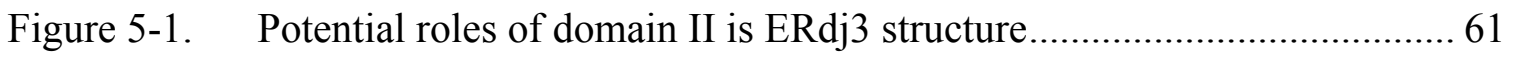

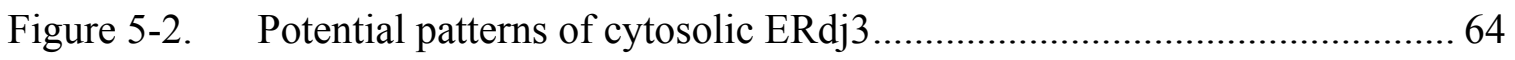

Figure 5-3. Model for dissociation of ERdj3 from substrate proteins ....................... 65

Figure A-1. Schematic drawings of the domain structure of Ydj1, Scj1 and ERdj3...84

Figure A-2. Expression of Ydj1 in mammalian ER ..............................................

Figure A-3. Expression of the various wild-type and mutant ERdj3 in yeast..............86

Figure B-1. WT/AJ ERdj3 do not bind to Protein A Sepharose beads by

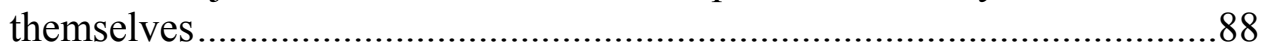

Figure B-2. Neither WT nor mutants BiP bind non-specifically to BSA blocked wells 


\section{CHAPTER 1. INTRODUCTION}

\subsection{Protein Folding}

Proteins are fundamental components of all organisms and participate in every process within cells. To be functional in cells, proteins have to fold and achieve their correct three-dimensional structure. The primary structure of a protein is a linear chain of amino acids that based on the properties of the amino acid side chains form a secondary structure of which the $\alpha$-helix and $\beta$-sheet are the most common ones. The amino acids that form the secondary structure then interact with each other and produce a completely folded, functional tertiary or in some cases quaternary structure.

Most folded proteins have a hydrophobic core inside the molecule formed by the side chains of the hydrophobic residues, which stabilize the protein, and charged or polar residues on the surface that interact with water molecules nearby. It is believed that the guiding principle of protein folding is to make the smallest number of hydrophobic sidechains exposed to the surface (Pace et al., 1996). Using both chemically or temperature denatured ribonuclease as the substrates, Christian Anfinsen showed that denatured ribonuclease could fold back to a functional shape by itself with the removal of denaturing chemicals or by lowering the temperature (Anfinsen, 1972). He concluded that all of the information required to fold a protein is encoded in the protein itself and that the amino acid sequence determines the shape of the protein. This discovery won him the Nobel Prize in Chemistry in 1972. Anfinsen's study was performed in vitro using a very dilute single protein, and folding started with a full length linear unstructured chain. Compared to in vitro conditions, the in vivo folding environment is much more crowded with many different types of nascent proteins, and folding often occurs co-translationally, so that the N-terminus of the protein begins to fold while the Cterminal portion of the protein is still being synthesized by the ribosome. In many cases, parts of the protein that will ultimately interact with each other come from very distant regions of the linear polypeptide chain.

\subsection{Molecular Chaperones}

Although proteins are able to achieve their native state on their own in isolation, it soon became appreciated that many of the proteins synthesized in cells needed additional help to either promote folding or prevent misfolding. The term "molecular chaperone" was first used by Ron Laskey in 1978 to describe the ability of nucleoplasmin, a nuclear protein, to prevent the aggregation of folded histone proteins with DNA during the assembly of nucleosomes (Laskey et al., 1978). Nucleoplasmin did not bind to nucleosomes or DNA, but instead was found to interact with histones to cover their positive charges. John Ellis extended the term in 1987 to define molecular chaperones as proteins that mediate the post-translational assembly of protein complexes (Ellis, 1987), but which are not themselves components of the final complex nor do they determine the final structure of a protein. However, once folding is complete the chaperones leave their client protein and move on to assist the folding of another protein. In 1988, it was 
determined that chaperones assisting in this process are conserved in both prokaryotes and eukaryotes (Hemmingsen et al., 1988).

There are two major families of chaperones that have been discovered: GroEL/GroES and Hsp70/DnaJs. GroEL is a highly conserved and ubiquitously expressed bacterial protein that is required for the proper folding of many proteins. To function properly, GroEL requires the assistance of the lid-like co-chaperone protein GroES. Within the cell, partially folded substrate proteins bind to a hydrophobic binding patch on the interior rim of the open cavity of GroEL and finish folding within this space (Martin et al., 1993). In eukaryotes, the mitochondrial proteins Hsp60 and Hsp10 are homologues of GroEL and GroES (Hartl, 1991; Tabibzadeh \& Broome, 1999) and TricC is a cytosolic orthologue, which functions independently of a small co-chaperone (Frydman et al., 1992).

Hsp70 is a large family of proteins that is highly conserved and homologues have been found in all organisms and all organelles. Hsp70 proteins were originally named based on their size of $\sim 70 \mathrm{kDa}$ and their induction by heat shock. The Hsp70 proteins are composed of an N-terminal nucleotide binding domain and a $\mathrm{C}$-terminal substrate binding domain. The main function of this group of proteins is to bind in a nucleotidedependent manner to relatively short extended regions on polypeptide chains and prevent them from aggregating. Using peptides to stimulate the ATPase activity of these proteins, it was discovered that peptides of $\sim 5-10$ amino acids in length that were relatively hydrophobic in nature were most likely to bind the Hsp70 protein (Flynn et al., 1989; Flynn et al., 1991). The recognition sequence was further defined using phage peptide display libraries (Blond-Elguindi et al., 1993; Fourie et al., 1994) or by screening cellulose bound peptides (Rudiger et al., 1997). These studies revealed that peptides composed of four or five amino acids with hydrophobic residues in alternating positions were mostly likely to bind. This was in keeping with the polypeptide chain being in an extended form with the hydrophobic residues pointing in the same direction (BlondElguindi et al., 1993). This idea was confirmed when a crystal structure was solved with a short peptide in the peptide binding domain of DnaK, the E. coli Hsp70 (Zhu et al., 1996). These binding sites are usually buried in the folded proteins and only available for the Hsp70 protein to bind when the substrate is in the unfolded form (Rudiger et al., 1997). Although the binding of GroEL and Hsp70 both rely on the hydrophobicity of the substrate, GroEL has a relatively low affinity for short peptides and prefers substrate peptides that have the capability to form some secondary structure such as an $\alpha$-helix, whereas Hsp70 recognizes completely unfolded regions of the substrate (Landry et al., 1992; Hayer-Hartl et al., 1994; Okazaki et al., 1994).

\subsubsection{Hsp70 proteins}

All of the Hsp70 proteins can be divided into four major domains. An N-terminal domain encodes a highly conserved $\sim 44 \mathrm{kD}$ nucleotide binding domain (NBD) that can bind ATP or ADP. A small flexible linker follows the NBD and links it to a $\sim 18 \mathrm{kD}$ substrate binding domain (SBD) that binds to unfolded polypeptides. The C-terminal portion is a less conserved $10 \mathrm{kD}$ lid domain, which is involved in capping the SBD. The 
NBD domain consists of two major lobes that form the nucleotide binding site. The substrate binding domain forms a $\beta$-sandwich, whereas the $10 \mathrm{kD}$ lid domain is mainly $\alpha$ helical (Zhu et al., 1996). The SBD contains a groove that can interact with the hydrophobic region on the unfolded or mis-folded proteins. All Hsp70 proteins have a weak ATPase activity, which is indispensable for binding to substrates. Binding of ATP or ADP regulates the interaction between Hsp70 and substrate. When the Hsp70 is in ATP bound form, the lid is open and allows peptides to bind to the SBD and release from it relatively rapidly. With the hydrolysis of ATP to ADP, Hsp70 undergoes a conformational change that causes the lid close allowing the SBD to associate with peptides tightly. The replacement of ADP with ATP induces another conformational change that reopens the lid and releases the substrate from the SBD (Munro and Pelham, 1986; Liberek et al., 1991; Buchberger et al., 1995). The ATPase cycle of Hsp70 is tightly controlled by regulators that either induce the ATPase activity (e.g. DnaJ proteins) or regulate nucleotide exchange (nucleotide exchange factors and nucleotide exchange inhibitors) (Figure 1-1). These co-chaperones are involved in the intramolecular communication between the ATPase and substrate binding domains and regulate association between Hsp70 and substrate (Freeman et al., 1995).

\subsubsection{Mammalian ER Hsp70}

Immunoglobulin heavy chain binding protein (BiP), which was found to associate with the unassembled, non-secreted Ig heavy chain produced in pre-B lymphoid cell lines, was the first identified chaperone of the endoplasmic reticulum (Haas \& Wabl, 1983). BiP interacts with various Ig assembly intermediates in plasmacytoma cells but not with completely assembled mature Ig molecules (H2L2) (Bole et al., 1986). Cloning of the mammalian BiP gene revealed that it was a member of the Hsp70 gene family (Munro et al., 1986; Haas \& Meo, 1988), suggesting that it was likely to play an important role in the folding and assembly of secretory pathway proteins. In addition to its role in protein folding and assembly (Gaut \& Hendershot, 1993; Simons et al., 1995; Lee et al., 1999; Vanhove et al., 2001), BiP contributes to ER calcium storage (Roy \& Lee, 1995; Lievremont et al., 1997), targeting mis- or unfolded proteins to the proteosome for degradation (Buchberger et al., 1995; Skowronek et al.,1998; Zhang et al., 2001; Kabani et al., 2003; Okuda-Shimizu and Hendershot, 2007), and regulating the UPR signal transducers (Bertolotti et al., 2000; Molinari et al., 2002;Shen et al., 2002). Furthermore, during early stages of translocation, BiP contributes to the maintenance of the permeability barrier of the ER translocon (Hamman et al., 1998), which is necessary to maintain a proper ER environment when nascent chains enter the secretory pathway (Figure 1-2). All of these BiP functions, except calcium storage, require its ATPase activity.

\subsection{Hsp40/DnaJ Protein}

Genetic studies isolated a number of genes in $E$. coli that are required for DNA replication of $\lambda$ phage. Two of these genes, DnaJ and DnaK, are required for both host cell and $\lambda$ phage DNA replication (Yochem et al., 1978). DnaJ is a $\sim 43 \mathrm{kD}$ protein that 


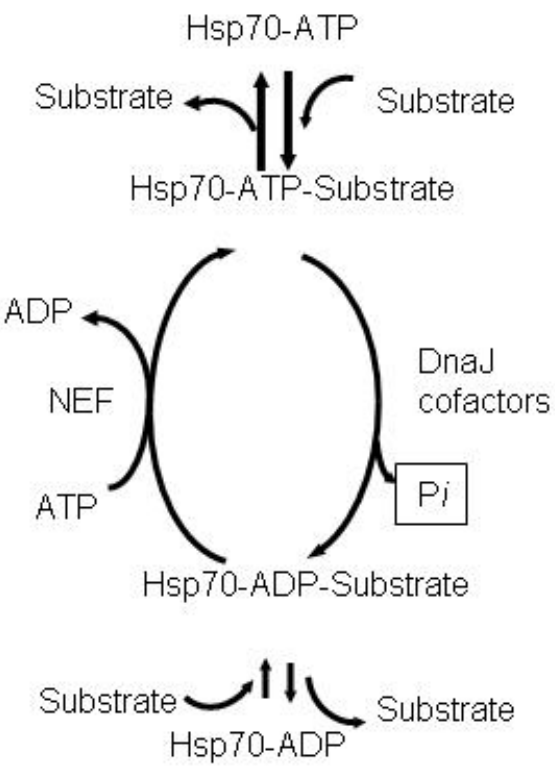

Figure 1-1. Hsp70s' ATPase cycle and substrate binding

Hsp70s alternate between ATP and ADP bound forms. Hsp70s associate and release substrate rapidly in ATP-bound form, whereas they bind and release substrate slowly in the ADP-bound form. DnaJ proteins stimulate the ATPase activity of Hsp70 and induce the hydrolysis of ATP to ADP. Nucleotide exchange factors (NEF) trigger the switch of ADP to ATP and reset the cycle. Nucleotide exchange inhibitors block the replacement of ADP with ATP. 


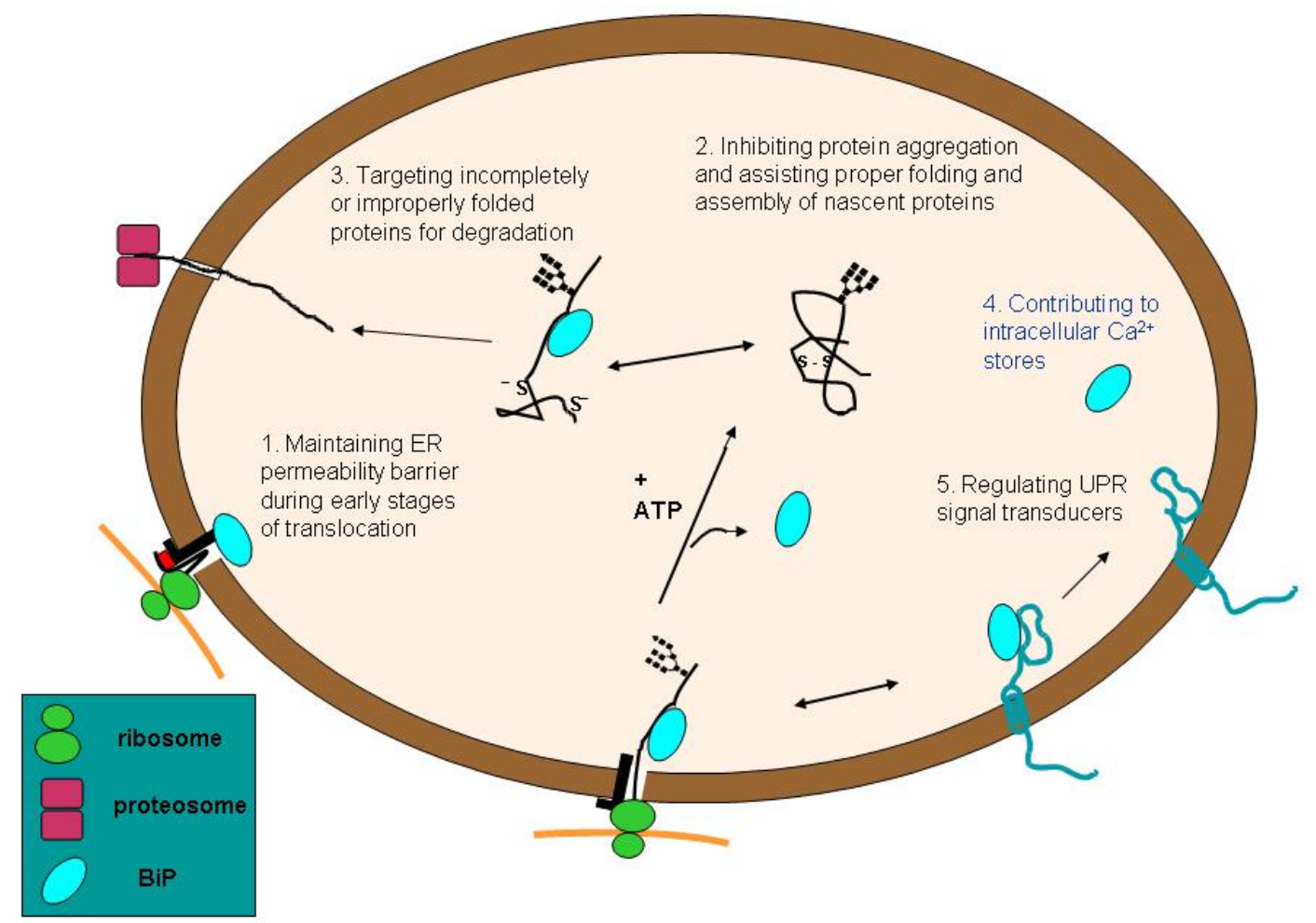

\section{Figure 1-2. BiP's multiple functions in ER}

$\mathrm{BiP}$ is involved in multiple processes in the mammalian ER including maintaining the permeability barrier of the ER translocon, assisting nascent polypeptide chain folding and assembly, targeting misfolded protein for degradation, contributing to ER calcium storage and sensing ER stress. All these functions except contributing to calcium storage require its ATPase activity which can be regulated by ER DnaJ proteins. 
can interact with DnaK and serve to stimulate the ATPase activity of DnaK, which is necessary for its chaperone activity (Liberek et al., 1991). Later studies recognized that many proteins possessed a domain that was very similar to the $\mathrm{N}$-terminal 70 amino acids of DnaJ, which was named the J domain, and proteins that had this domain were called DnaJ proteins (Cheetham \& Caplan, 1998; Walsh et al., 2004). Like Hsp70, DnaJ proteins are found in all organisms and organelles, but the number of DnaJ proteins far exceeds the Hsp70 proteins in any given organelle. For example, in the mammalian ER, 6 DnaJ proteins have been identified, whereas only one Hsp70 is present.

Based on their domain conservation with E. coli DnaJ, DnaJ proteins can be divided into three classes (Hennessy et al., 2000). Class I DnaJ proteins have the highest domain homology with $E$. coli DnaJ and possess all four domains. The N-terminal region encodes the $\sim 70$ amino acid J domain (Bardwell et al., 1986). There is a hallmark HisPro-Asp (HPD) tri-peptide motif in the middle of J domain (Liberek et al., 1991; Cheetham et al., 1998), which plays a key role in the interaction with DnaK (Wall et al., 1994). A glycine/phenylalanine-rich flexible domain (G/F) follows the J domain. The $C$ terminus includes a cysteine-rich domain containing four CysXXCys motifs that form two Zn2+ atoms binding sites (Banecki et al., 1996; Szabo et al., 1996) and the substrate binding domain (Liberek et al., 1990). The Gly/Phe region is not only the linker between $\mathrm{J}$ and C-terminal domains, but also is crucial for J domain's interaction with Hsp70 and for DnaJ function. Recently, crystallographic studies on the C-terminal fragment of Ydj1, a type I DnaJ protein in the yeast cytosol, revealed that the Cys-rich domain is a separate domain that is encoded within the linear sequence of the substrate binding domain (Li et al., 2003). The two halves of the substrate binding domain fold together to form a substrate binding pocket (Li \& Sha, 2005) (Figure 1-3). Class II DnaJ proteins have an N-terminal J domain and the Gly/Phe-rich linker, but lack the Zn2+ binding domain. The substrate binding domain of Sis1, a yeast cytosolic class II DnaJ protein was crystallized without a peptide substrate (Sha et al., 2000). Although its amino acid homology it not high with the substrate binding domain of Ydj1, they both share a very similar tertiary structure. Class III proteins only contain a J domain, which can occur anywhere in the protein. Some class III proteins also bind to substrate, although in no case has the substrate binding domain been clearly defined. For example, auxilin which contains a C-terminal J-domain can bind to clathrin and assists the uncoating of clathrincoated vesicles (Gruschus et al., 2004). SV 40 large T-antigen contains an N-terminal J domain and binds to p53 directly (Peden et al., 1989; DeCaprio, 1999). P58IPK, a recently identified mammalian ER DnaJ protein can interact with newly synthesized secretory proteins in vitro (Rutkowski et al., 2007) and possesses a C-terminus J domain (Melville et al., 1997). Generally, class I and II proteins share a higher level of conservation in the J domain than class III proteins do. Since they have less conserved J domains and lack a Gly/Phe-rich and Cys-rich regions, class III proteins may require higher specificity for their Hsp70 partner and substrates and only recognize a restricted subset of substrates. 


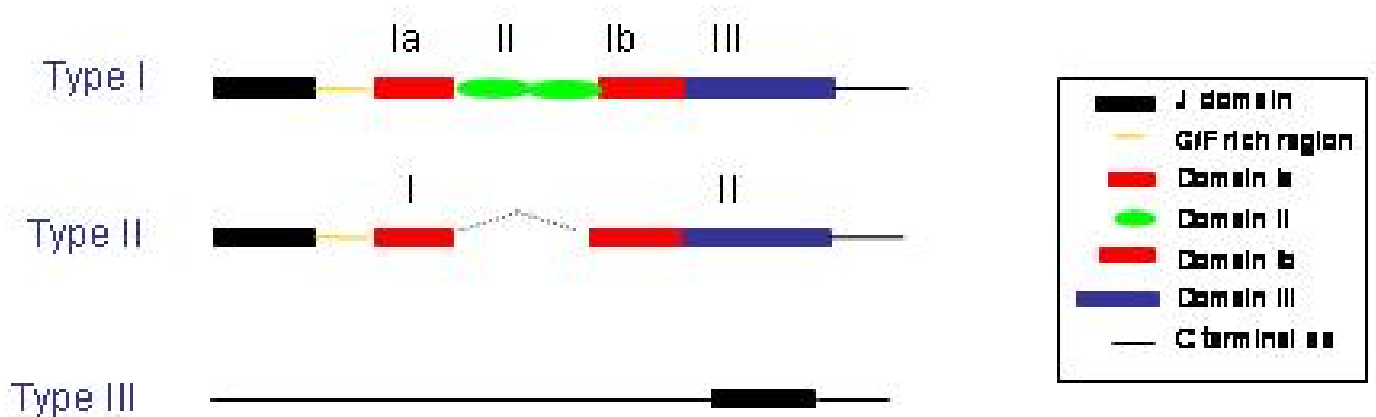

Figure 1-3. Three types of DnaJ family members

DnaJ proteins are divided into 3 subgroups, according to the domain conservation with E.coli DnaJ which consists of $\sim 70$ amino acid J domain (HPD motif), Gly/Phe rich domain, Cys-rich $\mathrm{Zn}^{2+}$ finger domain (domain II) and C-terminal domain. Type I and II DnaJ proteins are similar except that type II lacks domain II. Type III only contains J domain which can occur anywhere in the molecule. Recent studies indicate that domain I which is bisected in type I DnaJ contains substrate binding site. 


\subsubsection{Yeast DnaJ homologues}

There are three DnaJ proteins in the yeast ER and thirteen in the cytosol (Cheetham \& Caplan, 1998). Among these three ER DnaJ proteins, Scj1p is a class I DnaJ protein, and the other two, Sec63p and Jem1p, belong to class III. Sec63p is a transmembrane protein with a J domain that faces ER lumen and cooperates with Kar2p, a yeast Hsp70, to translocate proteins into the ER co- and post-translationally (Rothblatt et al., 1989; Sadler et al., 1989; Brodsky et al., 1995; Corsi \& Schekman, 1997). Unlike Sec63p, which is required for cell growth, Scj1p (Schlenstedt et al., 1995) and Jem1p (Nishikawa \& Endo, 1997) are soluble ER luminal proteins that are not essential for cell viability under normal growth conditions. However, loss of both JEM1 and SCJ1 causes a slow growth phenotype at elevated temperatures. Scj1p interacts with Kar2p to assist protein folding and assembly in the ER lumen (Schlenstedt et al., 1995). The fact that the $\Delta$ Scj1 mutant is hypersensitive to tunicamycin and mutations that block N-linked glycosylation, is consistent with Scj1's role in assisting protein folding (Silberstein et al., 1998). Jem1p interacts with Kar2p to mediate nuclear membrane fusion (Nishikawa \& Endo, 1997). In the yeast cytosol, there are thirteen DnaJ proteins identified that form distinct DnaJ/Hsp70 pairs to assist in Hsp70's various chaperone functions (Sahi \& Craig, 2007). One of them, Ydj1, a class I DnaJ protein, binds to the cytosolic side of the ER membrane through its C-terminus farnesylation site and is required for cell growth (Caplan \& Douglas, 1991). Sis1, an essential gene that cannot be replaced by Ydj1 (Luke et al., 1991), belongs to the class II subgroup (Sha et al., 2000; Li et al., 2005).

\subsubsection{Mammalian ER DnaJ homologues}

In the mammalian ER, six DnaJ proteins have been identified and named ERdj1-6. All of them interact with BiP through their J domain and stimulate its ATPase activity in vitro. ERdj1/Mtj1, is a transmembrane ER protein that binds the ribosome through its cytosolic domain and interacts with BiP through its luminal domain and is thought to couple translation with folding (Brightman et al., 1995; Chevalier et al., 2000; Dudek et al., 2002) and ERdj2/hSec63 (Skowronek et al., 1999; Tyedmers et al., 2000), the mammalian homologue of Sec63, is a multi-transmembrane ER proteins that belongs to the class III subgroup. Both of these proteins are associated with Sec61 and Sec62 and act as co-chaperones of $\mathrm{BiP}$ to translocate nascent polypeptides into the ER. ERdj3/HEDJ (Yu et al., 2000; Lau et al., 2001; Meunier et al., 2002), a 43 kD ER lumenal soluble DnaJ protein, was first identified as a shiga toxin binding protein. Shiga toxin is endocytosed from the plasma membrane and recognized by the cell as an unfolded protein. As such it is transported back to the ER and then is retrotranslocated to the cytosol to perform its function (Sandvig et al., 1992; Johannes \& Goud, 2000). Independently, ERdj3 was identified as a component of the chaperone complex that binds to free Ig heavy chains in mouse plasmacytoma lines (Meunier et al., 2002). ERdj3 is induced by ER stress, up-regulated during B cell differentiation and is most highly expressed in secretory tissues (Shen \& Hendershot, 2005; Shen \& Hendershot, 2007). These data suggest that ERdj3 is likely to serve as a cofactor for BiP in regulating protein folding, preventing aggregation, or targeting proteins for degradation. The fact that in ERdj3 knock out cells, the turn-over of free HC is increased argues that ERdj3 is most 
probably involved in protein folding and preventing aggregation (Shen et al., unpublished data). Homologues of ERdj3 are found in all metazoans and are highly conserved. ERdj3 has been suggested to be the orthologue of yeast Scj1, since both are ER lumen proteins and have similar size. ERdj4/Mdj1 (Prols et al., 2001; Shen et al., 2002) is a trans-membrane protein with its uncleaved signal peptide inserted into the ER membrane. The rest of the protein resides inside the ER where its $\mathrm{J}$ domain interacts with BiP. Like ERdj3, ERdj4 has the highest expression level in secretory tissues and is induced by ER stress (Shen et al., 2002). ERdj5, which contains four protein-disulfide isomerase domains (Cunnea et al., 2003; Hosoda et al., 2003) is a ubiquitous, abundant protein in the ER of cells. Recent data suggests that it may play role in either disulfide bond formation or reduction in the ER (Dong et al., 2008). ERdj6/P58IPK (Rutkowski et al., 2007 ) is the most recently identified member of the ER DnaJ family of proteins and is induced by ER stress and facilitates protein maturation (Figure 1-4).

\section{$1.4 \quad$ Nucleotide Exchange Factors}

To release the substrate from Hsp70 proteins, ADP must be released and ATP reinserted into the nucleotide binding domain. GrpE is a nucleotide exchange factor (NEF) found in bacteria, which binds to the ATPase domain of DnaK, induces the replacement of ADP with ATP, and triggers the release of unfolded substrates (Liberek et al., 1991; Szabo et al., 1994). Mge1p, a soluble mitochondrial matrix protein in $S$. cerevisiae is a GrpE homolog, acting in concert with mitochondrial hsp70 in protein translocation (Laloraya et al., 1994; Westermann et al., 1995). GrpE homologues also have been found in mammalian mitochondria that bind to mitochondria Hsp70 in an ADP dependent manner (Bolliger et al., 1994; Naylor et al., 1995; Naylor et al., 1998). SSE1 is the yeast homolog of mammalian NEF Hsp1 10 and acts as an efficient NEF for yeast cytosolic Hsp70 (Raviol et al., 2006). Although GrpE homologues are not present in other eukaryotic organelles, a number of both positive and negative regulators of nucleotide exchange have been identified. Hip binds to the ATPase domain of Hsc70 and stabilizes it in the ADP-bound state (Hohfeld et al., 1995; Prapapanich et al., 1996). BAG-1, a Bcl-2 binding anti-apoptotic factor was found to be able to promote the exchange of ADP to ATP in Hsp70 (Hohfeld \& Jentsch, 1997; Stuart et al., 1998). HspBP1 enhances nucleotide exchange for Hsp70 in vitro (Raynes \& Guerriero, Jr., 1998). Structural studies of GrpE with the ATPase domain of DnaK (Harrison et al., 1997) and the Bag-1/Hsc70 complex (Sondermann et al., 2001) provided insights into the mechanism of nucleotide exchange. Binding of the exchange factors to the ATPase domain of their Hsp70 triggers the release of substrate from Hsp70, which is achieved through the conserved conformational change in the ATPase domain of Hsp70.

A genetic screen identified the first nuclear exchange factor for the yeast ER Hsp70, Kar2p. SLS1 interacts directly with the ATPase domain of Kar2p and stimulates ATP hydrolysis by promoting ADP release in yeast Yarrowia lipolytica (Boisrame et al., 1998; Kabani et al., 2000; Tyson \& Stirling, 2000). Per100p (Travers et al., 2000) and Sillp (Tyson \& Stirling, 2000) are the homologues of SLS1 in S. cerevisiae. Using the ATPase domain of a BiP mutant as bait in a yeast two-hybrid screen, BAP (BiPAssociated Protein) was indentified as a nucleotide exchange factor for $\mathrm{BiP}$ in the 


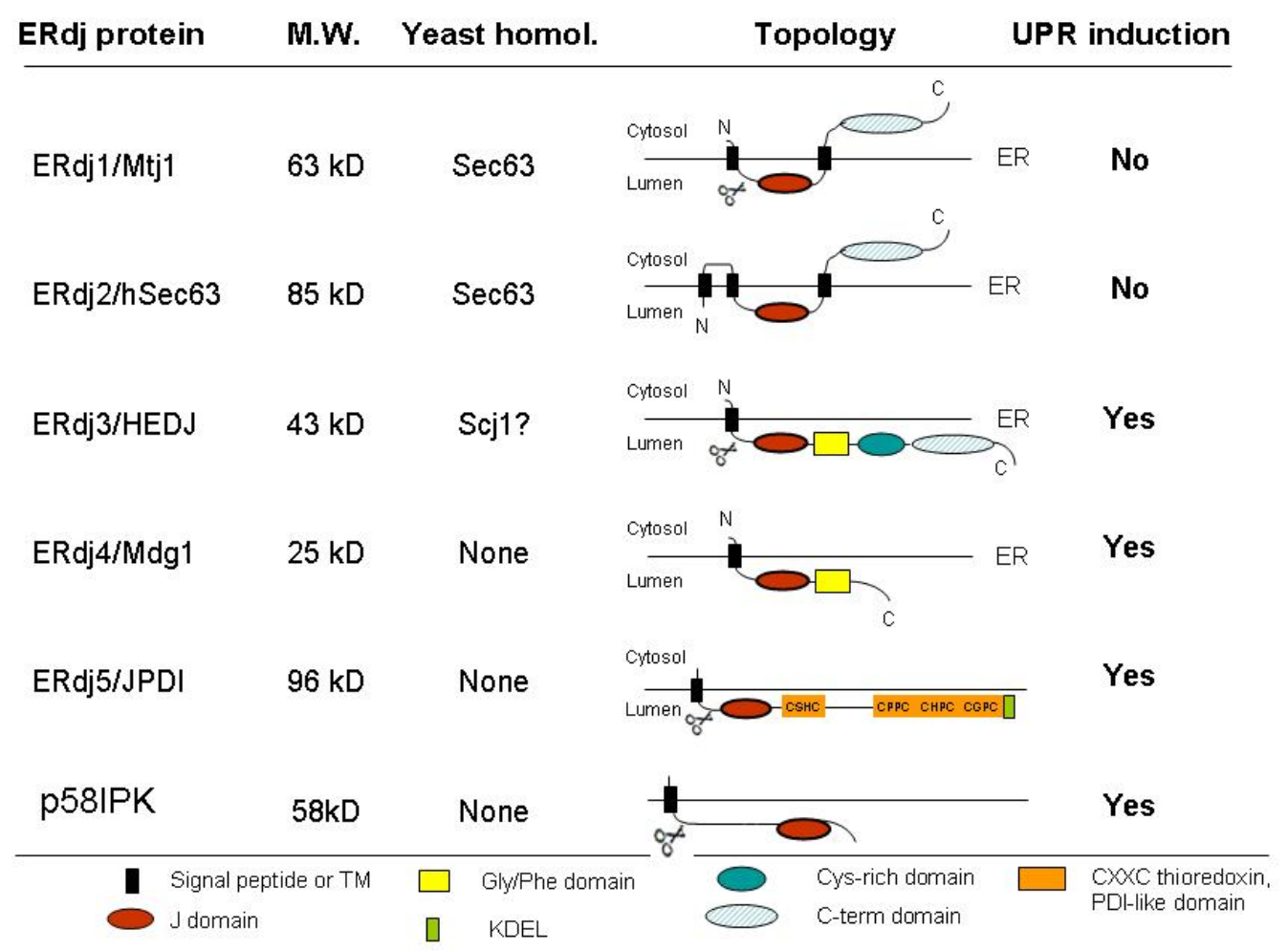

\section{Figure 1-4. Characterization of mammalian ER DnaJ proteins}

Six DnaJ proteins are identified in mammalian ER and named ERdj1-6 according the order they are found. 
mammalian in ER (Chung et al., 2002). BAP is an ER-localized glycoprotein that shows the highest expression levels in secretory pathway tissues. Although BAP releases both ATP and ADP from BiP in vitro, BAP prefers the ADP-bound form of BiP (Chung et al., 2002), which may allow BAP to drive the ATPase cycle of BiP forward.

In the yeast ER, Lhs1p was shown to be able to substitute for Sillp and to represent an alternative nucleotide exchange activity. Lhs 1 is a member of what has been referred to as the large Hsp70 family of proteins (Craven et al., 1996). These proteins show a high degree of homology at their N-terminus to Hsp70 proteins but contain large Cterminal extensions. GRP170, a mammalian orthologue of Lhs1p, has nucleotide exchange activity for BiP in vitro (Dierks et al., 1996), suggesting it can be an alternative exchange factor for BiP. In some mammalian cells, the function of BAP/Sill appears to be essential. Mutations that cause the loss function of Sill have been identified in humans and cause Marinesco-Sjögren syndrome, an autosomal dominant neurodegenerative disease (Senderek et al., 2005; Anttonen et al., 2008). A SIL1 knockout mouse or "woozy" mouse has been generated, which shows similar evidence of neurodegeneration (Zhao et al., 2005). The relationship between SIL1 mutations and Marinesco-Sjögren syndrome indicates that dysfunction of chaperone assisted protein folding in the endoplasmic reticulum can cause multisystem disorders (Sondermann et al., 2001; Senderek et al., 2005; Anttonen et al., 2008).

\subsection{Protein Folding in the ER}

The endoplasmic reticulum (ER) is a membrane-enclosed organelle found in all eukaryotic cells. The ER provides a specialized environment for the synthesis, folding, and assembly of nearly all proteins destined for secretion and cell surface expression. Secretory pathway and ER resident proteins are synthesized on ER membrane associated ribosomes in the cytosol. A hydrophobic signal sequence ( 11-20 amino acids), which usually occurs on the $\mathrm{N}$-terminus of the nascent polypeptide chain, is required to direct it to the translocon and allow it to be translocated into ER (Lingappa et al., 1978). After entering the ER, the signal sequence of the nascent polypeptide is often removed. Subsequently, glycans can be added to the nascent chain after $\sim 14$ amino acids have entered the lumen of the ER. Oligosaccharyl-transferase (OST) can co-translationally add N-linked glycans to asparagine residues in N-X-S/T site where asparagine is followed by any amino acid except proline and then a serine or threonine (Whitley et al., 1996). The OST complex is associated with the translocon and N-linked glycosylation serves to monitor the folding of the nascent chain. Nascent unfolded polypeptides are highly concentrated in the ER of a secretory cell. Unlike most organelles, an unusually high proportion of proteins in the ER are unfolded proteins or unassembled subunits that will ultimately be delivered out of ER for further transport along the secretory pathway once they are mature.

The ER environment is also unique in that it provides additional advantages and exerts certain restrictions to protein folding and assembly. The ER possesses an oxidizing environment and contains a group of enzymes that allow and assist the formation of disulfide bonds between adjacent cysteine residues (Hwang et al., 1992). 
The intra- and inter-chain disulfide bonds serve to stabilize folded regions of the nascent chain and assemble subunits and can form co-translationally. The nascent chain can fold co- or post-translationally inside the ER (Bergman \& Kuehl, 1979; Chen et al., 1995), and in some cases, assembly begins when the individual chains are still being synthesized (Bergman \& Kuehl, 1979). However, improper intra- and inter-chain disulfide bonds can form, which can lead to large, insoluble protein aggregates. Folding intermediates that contain improper disulfide bonds formed during synthesis must undergo isomerization to achieve the proper conformation. In addition, ER sequesters high concentrations of calcium, which is required for many signal transduction pathways (Lee, 1987) and clearly impacts normal protein folding in this organelle. The ER is also a unique environment for the production and storage of glycogen, steroids, and other macromolecules.

\subsection{ER Quality Control and ER Stress}

The ER is a crowded environment of newly synthesized, partially folded polypeptide chains where chaperones and folding enzymes aid and monitor the successful maturation of secretory pathway proteins through a process termed ER quality control. This tightly controlled system has set up stringent principals to ensure that only properly folded protein are delivered out of ER for secretion, while proteins that do not meet these requirements are quarantined in the ER (Hammond \& Helenius, 1995; Ellgaard et al., 1999). Incompletely folded nascent polypeptide chains in the ER are recognized and bound by two major molecular chaperone families before they are allowed to leave ER for the secretory pathway: the Hsp70 family member (BiP), and lectin-like proteins, calnexin and calreticulin (Ellgaard \& Helenius, 2001). The calnexin/calreticulin chaperones system recognizes the monoglucosylated, N-linked glycans and unfolded regions on nascent glycoproteins (Sousa et al., 1992; Trombetta \& Parodi, 1992; Hammond et al., 1994). Cleavage of this glucose by glucosidase II (Kornfeld \& Kornfeld, 1985) blocks the binding of calnexin/calreticulin and properly folded proteins are allowed to leave the ER (Trombetta \& Parodi, 1992; Hebert et al., 1995). However, incompletely folded or mis-folded peptides will be recognized by UDPglucosyltransferase (GT), an ER resident protein, which binds to unfolded regions of the nascent protein and adds a glucose to a nearby glycan allowing the unfolded substrate to reenter the cycle (Sousa et al., 1992; Hebert et al., 1995). So this system is dependent on the presence of both unfolded regions and glycans. The Hsp70 (BiP) chaperone family recognizes the hydrophobic residues on the unfolded region of nascent peptides (Flynn et al., 1991; Blond-Elguindi et al., 1993). When N-linked glycosylation is blocked, in some cases BiP can recognize calnexin/calreticulin substrates (Balow et al., 1995; Zhang et al., 1997).

Unfolded or misfolded proteins that cannot bury hydrophobic regions by completing their folding remain associated with these chaperones and are retained in the ER. Because calnexin is a resident ER integral membrane protein, its binding to substrates serves to retain them in this organelle. Calreticulin and $\mathrm{BiP}$ are soluble proteins that are themselves kept in the ER due to an ER retention sequence at their Cterminus (Munro \& Pelham, 1987). The binding of these molecular chaperones keeps unfolded proteins in the ER, prevents them from aggregating, and provides them with 
additional opportunities to achieve their correct conformation. Proteins that ultimately fail to fold and assemble properly are retrotranslocated from the ER to the cytosol for intracellular degradation by the 26S proteasome (Werner et al., 1996; Hampton, 2002; Jarosch et al., 2003). The assembly of individually synthesized protein subunits in the ER is also monitored and regulated by this quality control system.

To deal with changes in the normal environment of ER that affect protein folding and lead to the accumulation of unfolded or misfolded proteins, ER chaperones and components of the quality control apparatus are up-regulated to bind and prevent proteins from aggregating, and provide them with additional opportunities to achieve their correct conformation. This response is termed unfolded protein response (UPR) which helps to relieve ER stress. The UPR can be induced by changing cell growth conditions (low $\mathrm{pH}$, hypoxia, glucose deprivation, and calcium imbalances) or by some pharmacological reagents that affect ER protein folding (tunicamycin, thapsigargin, and DTT). The UPR is regulated through the activation of three ER transmembrane stress transducers: IRE1 (Ire $1 \alpha$ and $\beta$ ), PERK (PKR-like ER kinase) and ATF6 (Ma \& Hendershot, 2004). Under normal conditions, BiP inactivates all three mammalian UPR transducers by binding to their lumenal domains. When unfolded proteins accumulate, $\mathrm{BiP}$ is released from the lumenal domains of the transducers. Both IRE1 and PERK dimerize or oligomerize and are phosphorylated in trans. Ire $1 \alpha$ and $\beta$ contain $C$-terminal endoribonuclease activity that is activated during ER stress to splice 26 bases from the XBP-1 (X-box binding protein) mRNA, which causes a shift in the reading frame of the 3 ' end of the mRNA to generate the active form of XBP-1 (sXBP-1). sXBP-1 protein in turn binds to UPR elements in the promoters of an number of UPR target genes including ERdj3 (Shen \& Hendershot, 2007), ERdj4 and EDEM ( Lee et al., 2002). The Ire1 pathway is conserved between yeast and mammalian cells. Ire 1p, the yeast homolog, remodels HAC1 mRNA by the same mechanism (Cox \& Walter, 1996). Activated PERK phosphorylates translation initiation factor eIF-2 $\alpha$, which inhibits cap-dependent translation and therefore general protein synthesis to reduce the accumulation of unfolded proteins (Harding et al., 1999). In the case of ATF6, dissociation of BiP allows the protein to be transported to the Golgi, where the S1P and S2P proteases cleave the membrane-bound ATF6 $(90 \mathrm{kD})$, allows the release of its cytosolic transcription-factor domain from membrane $(50 \mathrm{kD})$. Then the active form of ATF6 is transported to nucleus where it binds and transactivates ER stress elements found in the promoters of ER chaperones and folding enzymes (Yoshida et al., 1998; Haze et al., 1999). ATF6 also transcriptionally upregualtes XBP-1 (Haze et al., 1999; Yoshida et al., 2000) which is regulated by Ire1 as described.

\subsection{DnaJ Proteins as Specifiers of Hsp70 Function}

In a given organelle, DnaJ proteins work with their Hsp70 partner to regulate multiple functions. The number of DnaJ proteins usually far exceeds the number of Hsp70s, suggesting that an individual DnaJ may be specific for different functions of Hsp70. BiP binds to unfolded or incompletely assembled proteins to assist their folding and assembly as well as to misfolded proteins that need to be degraded. In addition $\mathrm{BiP}$ seals the translocon and regulates the UPR signal transducers. Since BiP has multiple 
functions, it is possible that various ERdjs specify and regulate the different functions of BiP and perhaps even more ERdjs will be identified.

Many DnaJ proteins can interact directly with unfolded substrates and prevent their aggregation in vitro. Although in most cases, the substrate binding region has not been identified, studies on E. coli DnaJ (Banecki, 1996; Szabo, 1996) and the yeast cytosolic DnaJ proteins, Ydj1 (Li et al., 2003) and Sis1 (Sha et al., 2000), have mapped the peptide binding region to the $\mathrm{C}$-terminal half of the protein. Although Ydj1 and Sis1 belong to different classes of DnaJ proteins and the amino acid conservation between them is quite low, the overall structure was remarkably similar. The substrate binding domain (domain I) of Ydj1 is nearly identical to that found in Sis1, except that in the case of Ydj1, domain

I is bisected in the linear sequence with a Cys-rich domain that folds independently and is named domain II. The structure of peptide binding fragment of Ydj1(aa 102-384) was solved with a peptide bound to a hydrophobic face on domain I that was composed of residues from both the Ia sequence and the Ib sequence.

\subsection{Summary}

Until now, the substrate binding characteristics of mammalian DnaJ proteins have been poorly understood. Since ERdj3 interacts with unassembled Ig heavy chains unusually stably in vivo, it provides a good model to study the interaction between a mammalian DnaJ protein and unfolded substrate. In our first study, we determined ERdj3's secondary structure (ProteinPredict) and modeled its tertiary structure on that of Ydj1. Using a combination of deletional analysis and point mutations, we characterized the structural requirements for its binding to substrates by using unassembled $\mathrm{HC}$ in in vivo assays and denatured luciferase in in vitro assays (Chapter 2).

Because we found that ERdj3 was structurally very similar to Ydj1, we further characterized the structural requirements for in vivo function, by testing the ability of Ydj1 to function in the mammalian ER using a transient expression system and for ERdj3 to complement yeast that were deficient in either Scj1, the ER DnaJ protein or Ydj1, the cytosolic family member. Because we found that ERdj3 could compensate the loss of Ydj1, this allowed us to examine the ability of various structural motifs that we demonstrated to be important for substrate binding (Chapter 3).

Finally, although the requirements for Hsp70 binding and release have been well characterized, it has not been clear how DnaJ proteins are released from substrates. Using a series of ERdj 3 and BiP mutants with denatured luciferase as the substrate, we determined the functional requirements for both BiP and ERdj3 that induce the release of ERdj3. This allowed us to propose a general model for the release of DnaJ proteins from substrates (Chapter 4). 


\section{CHAPTER 2. ERDJ3, A LUMENAL ER DNAJ HOMOLOG, BINDS DIRECTLY TO UNFOLODED PROTEINS IN THE MAMMALIAN ER: IDENTIFICATION OF CRITICAL RESIDUES}

\subsection{Introduction}

Hsp70 proteins are a family of molecular chaperones found in all organisms and all organelles. BiP is the mammalian ER Hsp70 homologue and was first identified as an immunoglobulin heavy chain binding protein (Haas \& Wabl, 1983; Bole et al., 1986). Like other Hsp70s, BiP contains an N-terminal nucleotide binding domain that can interact with ATP or ADP (Kassenbrock \& Kelly, 1989) and a C-terminal substrate binding domain (Hendershot et al., 1995). The ATPase activity of Hsp70 proteins is essential for their ability to bind to substrates. In the ATP-bound state, the substrate binding domain (SBD) is open, which results in both a fast on and off rate for interaction with unfolded proteins. In the ADP-bound state, the SBD is closed and binds to substrates slowly but tightly (Liberek et al., 1991). Binding of unfolded proteins to the SBD stimulates Hsp70's ATPase activity and induces the hydrolysis of ATP to ADP in vitro (Flynn et al., 1989). During this process, Hsp70 protein undergoes a conformational change, which induces closure of a lid over the SBD and stabilizes the interaction between Hsp70 and the substrate. The release of ADP and rebinding of ATP resets the Hsp70 to the "open" form, which allows the substrate to be released and to fold (Munro et al., 1986; Liberek et al., 1991; Buchberger et al., 1995).

The ATPase cycle of Hsp70 proteins is regulated by co-factors that either induce ATP hydrolysis or regulate nucleotide exchange. DnaJ proteins interact with the ATP bound form of Hsp70s and induce ATP hydrolysis (Liberek et al., 1990). To date, more than 100 proteins have been designated as DnaJ-like proteins due to the presence of a highly conserved $\sim 70$ amino acid domain that has been termed the "J domain". The $\mathrm{J}$ domain contains the signature His-Pro-Asp (HPD) tri-peptide motif, which plays a critical role in the interaction with Hsp70 (Liberek et al., 1991). Mutation of HPD to either QPD or HPN abolishes the interaction between the mutant $\mathrm{J}$ domain and its Hsp70 partners (Wall et al., 1994; Suh et al., 1998; Tsai et al., 2002). DnaJ proteins can be divided into three subgroups according to their domain conservation with $E$. coli DnaJ (Hennessy et al., 2000). Like E. coli DnaJ, Type I DnaJ proteins contain four domains: an $\mathrm{N}$ terminal J domain, a Gly/Phe-rich flexible linker domain, followed by a Cys-rich region that forms two $\mathrm{Zn}^{2+}$ binding sites (Banecki et al., 1996; Szabo et al., 1996), and a $\mathrm{C}$ terminal domain that may contain substrate binding motif (Liberek et al., 1990). Type II DnaJ proteins are similar to type I proteins, except that they lack the Cys-rich region. Type III DnaJs possess only the J domain, which can be present anywhere in the molecule. Like Hsp70s, DnaJ proteins exist in all organelles and organisms, but often far out number the Hsp70s present. Type I and type II DnaJ proteins can interact directly with unfolded substrates and inhibit protein aggregation in vitro. Studies on E. coli DnaJ (Banecki et al., 1996; Szabo et al., 1996) and the yeast cytosolic DnaJ proteins, Ydj1 (Li et al., 2003; Li \& Sha, 2005) and Sis1 (Sha et al., 2000), have further defined the peptide binding region of type I and II DnaJ proteins. Although some Type III DnaJs bind to substrate directly, like auxilin, which contains a C-terminal J-domain and binds to 
clathrin to assists in the uncoating of clathrin-coated vesicles (Gruschus et al., 2004), there are no structural data available for any of these family members.

Six mammalian ER localized DnaJ-like proteins have been identified and named ERdj1-6 (Brightman et al., 1995; Skowronek et al., 1999; Yu et al., 2000; Prols et al., 2001; Shen et al., 2002; Cunnea et al., 2003; Hosoda et al., 2003; Rutkowski et al., 2007). ERdj3 is a soluble, lumenal DnaJ family member that binds to unassembled immunoglobulin heavy chains (HC) along with the BiP chaperone complex in the ER (Meunier et al., 2002) and with a number of other unfolded proteins that are BiP substrates (Shen \& Hendershot, 2005). The demonstration that the ERdj3 J domain mutant (HPD $\rightarrow$ QPD), which abolished its interaction with BiP, did not affect its ability to bind to substrate (Shen \& Hendershot, 2005) led us to hypothesize that ERdj3 might bind directly to unfolded substrates. Since the interaction between ERdj3 and unassembled heavy chains is unusually stable in vivo, it was a particularly good model to investigate the structural features of ERdj3 that are important for substrate binding. Using secondary structure predictions and tertiary structure modeling, we found that the C-terminus of ERdj3 is very similar to that of Ydj1 (Li et al., 2003). The domain I sequence is interrupted with a domain II, although it is much smaller and has an entirely different sequence than that of Ydj1. Like domain I of Ydj1, it was found to be important for substrate binding. Mutation of conserved hydrophobic residues in domain I significantly reduced interaction with substrates in vitro. In addition, we demonstrated that ERdj3 exists as a dimer in cells and found that Phe326 plays a critical role in dimerization, which also affected substrate binding. Finally, our data revealed that domain II was essential for association with $\gamma \mathrm{HC}$ and denatured luciferase.

\subsection{Materials and Methods}

\subsubsection{Production of ERdj3 mutants}

ERdj3 mutants were made using a Quick Change Site-Directed Mutagenesis PCR Kit (Stratagene) with wild type HA-tagged ERdj3 (3HA-DSL-ERdj3) and QE-ERdj3 serving as the templates. The QPD mutant was generated previously in our lab (Shen et al., 2005). Other mutants were generated by PCR using the indicated primer pairs.

$\triangle \mathrm{J}$ : 5'primer GGGGCGGTGATTGCCAAAGATGGTCATCAG and 3' primer CTGATGACCATCTTTGGCAATCACCGCCCC $\triangle \mathrm{G} / \mathrm{F}$ : 5'primer GGTGAAGAAGGATTACCTCGTCAGCAAGAC and 3'primer GTCTTGCTGACGAGGTAATCCTTCTTCACC $\triangle$ Ia: 5'primer CATGTTTGGAGGAACCAAGTGCAATTGTCGGC and 3'primer GCCGACAATTGCACTTGGTTCCTCCAAACATG $\triangle$ G/FAIa: 5'primer GGTGAAGAAGGATTAAAGTGCAATTGTCGGC and 3'primer GCCGACAATTGCACTTTAATCCTTCTTCACC III: 5'primer GTTAGAAACAAACCTGTGGCA-CTAGTGAATGAAGAACGAACG and 3'primerCGTTCGTTCTTCATTCACTAG-TGCCACAGGTTTGTTTCTAAC 
$\triangle$ IIGSGG:5'primerGTTAGAAACAAACCTGTGGCAGGCAGCGGGGGCCTAGTGA ATGAAGAACGAACG and 3'primer

CGTTCGTTCTTCATTCACTAGGCCCCCGCTGCCTGCCACAGGTTTGTTTCTAAC I113A: 5'primer CCAAGAGGAAGTGATGCTATTGTAGATCTAGAAGTC and 3' primer GACTTCTAGATCTACAATACCATCACTTCCTCTTGG V132A: 5' primer GCAGGAAATTTTGTGGAAGCAGTTAGAAACAAACCTGTGG and 3' primer CCACAGGTTTGTTTCTAACTCCTTCCACAAAATTTCCTGC L226A: 5' primer GTGAATGAAGAACGAACGGCGGAAGTAGAAATAGAGCCTG and 3' primer CAGGCTCTATTTCTACTTCCCCCGTTCGTTCTTCATTCAC F241A: 5' primer CCTGGAGATTTACGGGCCCGAATCAAAGTTGTC and 3' primer GACAACTTTGATTCGGGCCCGTAAATCTCCAGG F326A: 5' primer GGGCTCTTTGATAATCACTTTTGATGTGGATGCTCCAAAAGA ACAG and 3' primer CTGTTCTTTTGGAGCATCCACATCAAAAGTGATTATCAAA GAGCCC

F326D: 5'primer GGGCTCTTTGATAATCACTTTTGATGTGGATGATCCAAAAGA ACAG and 3'primer CTGTTCTTTTGGATCATCCACATCAAAAGTGATTATCAAA GAGCCC

To generate the $\triangle \mathrm{III}$ mutant, a PCR reaction was performed using 3HA-DSLERdj3 as the template with 5'EcoRI-CGGAATTCGGACCCGGGAC and 3'NotIATAAGAATGCGGCCGCCAGTGCTTGACAACTTTGATTCGG as primer pairs. After cutting the PCR product with EcoRI and NotI, it was ligated into the 3HA-DSL vector in place of the corresponding EcoRI - NotI fragment present in full length ERdj3. This mutant removes all of domain III as well as the remaining $30 \mathrm{C}$-terminal amino acids.

\subsubsection{Cell culture, transfection and immunoprecipitation}

COS-1 monkey kidney fibroblast cells were maintained in DMEM media supplemented with 10\% fetal bovine serum, $2 \mathrm{mM}$ L-glutamine, and 1\% Fungisone and cultured in $3 \% \mathrm{CO}_{2}$ at $37{ }^{\circ} \mathrm{C}$. Cells were transfected with the indicated vectors using the Fugene 6 transfection reagent (Roche Diagnostics), and forty-eight hours posttransfection cells were labeled with ${ }^{35} \mathrm{~S}$ Translabel (Amersham Biosciences) for $3 \mathrm{~h}$. After washing with PBS, cells were incubated with 3,3'-dithio-bis (propionic acid Nhydroxysuccinimide ester) (DSP), a membrane permeable cross-linking reagent as described previously (Meunier et al., 2002a) to stabilize the interaction of ERdj3 with HC. Cell lysates were prepared using an NP40 lysing buffer and immunoprecipitated with the indicated antisera followed by binding to Protein A Sepharose beads. The rabbit polyclonal ERdj3 antibody (Shen et al., 2005) was affinity purified on recombinant mouse ERdj3 protein for our studies. All the ERdj3 deletion constructs were engineered with an HA-epitope tag at their $\mathrm{C}$ terminus, which can be recognized with an anti-HA monoclonal antibody (a kind gift of Dr. Albert Reynolds, Vanderbilt University). Immunoprecipitated complexes were analyzed by SDS-PAGE under reducing conditions, and the signal was detected using Amplify (Amersham Bioscience) for autoradiographic visualization. 


\subsubsection{Protein purification}

The expression of His-tagged wild-type and mutant ERdj3 proteins were induced in E. coli M15 cells with $0.1 \mathrm{mM}$ isopropyl $\beta$-D-thiogalactoside (Sigma) followed by growth for $18 \mathrm{~h}$ at $18^{\circ} \mathrm{C}$. The recombinant proteins were purified on $\mathrm{Ni}^{2+}$-agarose columns under non-denaturing conditions (Qiagen QIAexpress system), dialyzed in $25 \mathrm{mM}$ sodium phosphate buffer $(\mathrm{pH} 7.0)$ containing $150 \mathrm{mM} \mathrm{NaCl}, 0.02 \%$ Triton $\mathrm{X}-100$, $50 \%$ glycerol, and a protease inhibitor cocktail (Roche), and stored at $-20^{\circ} \mathrm{C}$.

\subsubsection{Measurement of complex formation between ERdj3 proteins and denatured luciferase}

Wild type or mutant ERdj3 recombinant proteins $(0.5 \mu \mathrm{g}$ in $100 \mu \mathrm{l}$ of PBS containing $0.05 \%$ BSA) were added to each well of a 96 -well microtiter plate purchased from Theromo (Immulon 2HB Flat Bottom Microtiter Plate) and allowed to bind overnight at $4^{\circ} \mathrm{C}$. Wells were washed three times with PBS to remove unbound ERdj3 and blocked with $200 \mu \mathrm{PBS}$ containing $1 \%$ BSA for $1 \mathrm{~h}$ at room temperature. Firefly luciferase was denatured in buffer containing 7M Urea, 25mM HEPES (pH 7.5), 50mM $\mathrm{KCl}, 5 \mathrm{mM}$ EDTA, $5 \mathrm{mM} \mathrm{MgCl} 2$, and $5 \mathrm{mM}$ dithiothreitol at room temperature for $40 \mathrm{~min}$ and then diluted into PBS containing $0.05 \%$ BSA (final concentration $5 \mu \mathrm{g} / \mathrm{ml}$ ). $100 \mu \mathrm{l}$ of this solution was added to the wells containing wild type or mutant ERdj3 and allowed to bind for $1 \mathrm{hr}$ at room temperature followed by washing with PBS to remove unbound luciferase. The amount of denatured luciferase that remained bound to ERdj3 was detected with a polyclonal anti-luciferase antiserum followed by donkey anti-rabbit Ig conjugated to alkaline phosphatase (Promega). The substrate 4-Nitrophenyl phosphate disodium salt hexahydrate (Sigma) was added to the wells for approximately $10 \mathrm{~min}$, and then $0.75 \mathrm{M} \mathrm{NaCl}$ was added to stop the reaction. The plates were read on a spectrophotometer (BioRAD) at wavelength 405nM. Negative controls were included for each plate in wells that did not contain either denatured luciferase, ERdj3, or each of the antibodies, but which included all the other steps of the reaction. The amount of DLuc that bind to WT ERdj3 was set as $100 \%$, the amount of D-Luc bound to indicated forms of mutants were set as percentage of WT.

\subsection{Results}

\subsubsection{Modeling ERdj3's secondary structure}

We have recently demonstrated that ERdj3 can bind directly to free $\mathrm{HC}$ and denatured luciferase in vitro (Jin et al., submitted). To begin to characterize the regions of ERdj3 that contribute to substrate binding, we first used a computer program (Predictproteins) to generate a secondary structure prediction for ERdj3 and compared it with those of two yeast cytosolic DnaJ proteins for which structural data are available for the protein binding domain, Ydj1 (type I DnaJ subgroup) and Sis1 (type II DnaJ subgroup) (Sha et al., 2000; Li et al., 2005). Although the amino acid sequences of these 
two proteins are not highly conserved, their overall secondary (Figure 2-1A) and tertiary structures are very similar, except that Sis1 lacks the cysteine-rich domain II, which is a hallmark domain of type I DnaJ proteins. Comparison of the secondary structure predictions revealed that ERdj3 is likely to be more similar to Ydj1, in that it appears to have an additional sequence (domain II) inserted within domain I (Figure 2-1A). However, the sequence and size of this domain is quite different from that of Ydj1 (Figure 2-1A \& B). The corresponding domain in Ydj1 is 65 amino acids in length, with 8 cysteine residues that form two zinc binding centers (CXXCXG motif), whereas the predicted domain in ERdj3 is only 40 amino acids in length and possesses only four cysteine residues that form two intradomain disulfide bonds (Shen et al., 2005; Marcus et al., 2007). Using PyMol (DeLano Scientific LLC ) to model the structure of ERdj3 in this region, we found that domains I and III of these two molecules appeared to be almost identical, whereas domain II could not be modeled on the structure of Ydj1 (data not shown).

\subsubsection{Mapping ERdj3's substrate binding domain in vivo}

Based on the crystal structures of Ydj1 and Sis1 and our ability to model ERdj3 on the Ydj1 structure, we defined domain boundaries for ERdj3 and referred to them as domain I (Ia and Ib), II and III and made corresponding deletion mutants (Figure 2-2A). In addition, we deleted the J domain, the Gly/Phe-rich domain alone, and the Gly/Pherich domain along with domain Ia to investigate the role of each of these domains in substrate binding. The HA-epitope tag that is encoded at the carboxyl terminus of each of these mutants ensured our ability to immunoprecipitate each of them. For domain III, we removed the sequence that was predicted to form this domain, as well as the remaining 30 amino acids at the carboxy-terminus. Domain Ia and $\mathrm{Ib}$ were deleted separately, even though the structural predictions suggest that they should fold together to form an intact domain I. In the case of domain II, two deletion mutants were made, one that removed only the sequence encoding this domain $(\Delta \mathrm{II})$ and a second one in which domain II was replaced by a four amino acid flexible linker, GSGG, to increase the possibility that the two halves of domain I would fold correctly in the absence of domain II (Figure 2-2A). Each of the ERdj3 constructs was first expressed in COS cells alone. SDS-PAGE analysis revealed that all of the proteins were expressed and could be immunoprecipitated with the anti-HA antibody (Figure 2-2C). Importantly none of these proteins bound non-specifically to Protein A Sepharose beads (Figure 2-2C). The ERdj3 constructs were next co-expressed with $\gamma \mathrm{HC}$, and an attempt was made to normalize the expression of the various mutants. To determine the ability of the various ERdj3 proteins to associate with $\gamma \mathrm{HC}$, cells were treated with DSP, which is required to stabilize ERdj3's association with $\gamma \mathrm{HC}$ (Meunier et al., 2002), and then co-immunoprecipitation assays were performed with the indicated antibodies (Figure 2-2B). The presence of $\gamma \mathrm{HC}, \mathrm{BiP}$, and GRP94 in the anti-HA lanes is not meaningful, as the $\gamma \mathrm{HC}$ bind directly to Protein ASepharose and co-precipitate these two chaperones. When each of the lysates was immunoprecipitated only with Protein A beads to isolate $\mathrm{HC}$, we found that wild-type ERdj3 was co-precipitated as expected (Figure 2-2B). Examination of each of the mutants revealed that deletion of the $\mathrm{J}$ domain $(\Delta \mathrm{J})$ actually increased the binding of this 
A.

\begin{tabular}{|c|c|c|c|}
\hline Ydjl & EEEEEEE & HHHHHHHHH & НHНHНHНHНHНHНHНHНHН НHHHHН \\
\hline EEEEEEEEHHH EEEEEEEEEEEE & EEEEEEEE & EEEEEEE & EEE EEEFEEEEEEEE \\
\hline EEEEEEEE EEE EEEEEEEEEHHHH & EEEEEE & EEEEEE & HHHHHHHHHH \\
\hline
\end{tabular}

\begin{tabular}{|c|c|c|c|c|}
\hline & & EEEEEE & НHНHНHНHНHНHНHНHНH & НННННННННННН НHННННННННН НННННННН \\
\hline $\begin{array}{l}\text { EEEEEEEEEEEEEE } \\
\text { EEE EEEEEEEEHH }\end{array}$ & EEEEEE & EEEEEE & $\begin{array}{l}\text { EEEEEE } \\
\text { EEEEEEEE }\end{array}$ & $\begin{array}{c}\text { EEEEEEEEE } \\
\mathrm{HHHHHHHH}\end{array}$ \\
\hline
\end{tabular}

\begin{tabular}{|c|c|c|c|c|}
\hline ERdj3 & & EEEEE & НHНHНHНHНHНHНHНHНHН & HНHНHНHНHНHНHНHНHНHH НHНHНHНH \\
\hline EEEEEEEEEEE & EEEEEEE EE & EEEEEE & EEEEEEEE & EEEEEEEEEEEEE EEEEEE \\
\hline EEEEEEEE EE & EEEEEEEEEHHHH & EEEEE & EEEEEE & $\mathrm{HHHHHHHHHHH}$ \\
\hline
\end{tabular}

B.

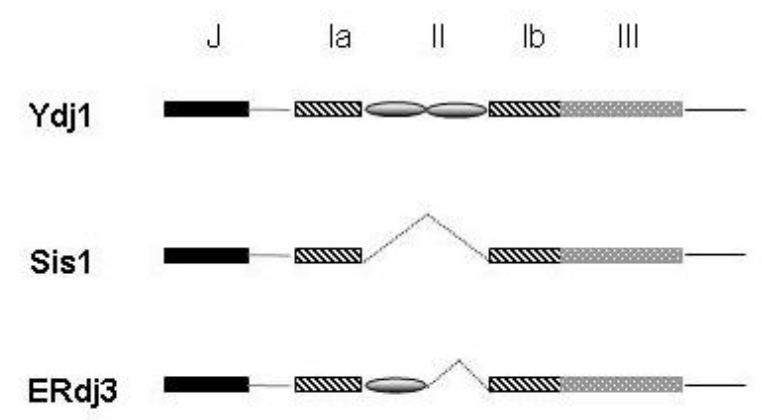

\section{Figure 2-1. Structural comparisons of the DnaJ proteins}

(A) Secondary structure predictions for Ydj1, Sis1, and ERdj3. Colors represent the different domains: Light grey: ER targeting sequence; Grey: J domain; Bold and Italics: domain I; Bold and underlined: domain II, and Black: domain III. H: $\alpha$ helix and E: $\beta$ sheet. (B) Shematic of domain boundaries for Ydj1, Sis1 and ERdj3. Sis1does not contain cysteine-rich domain II found in Ydj1. ERdj3 is more similar to Ydj1, although it contains an atypical, smaller domain II. 
A.

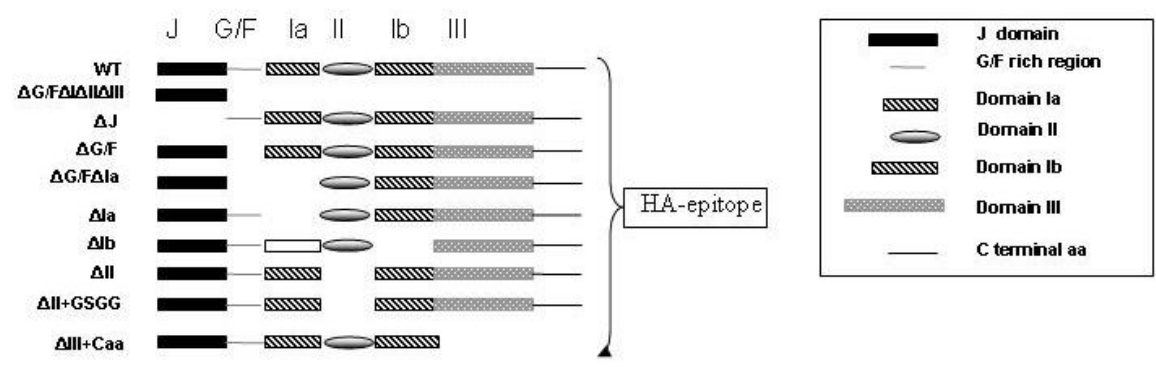

B.

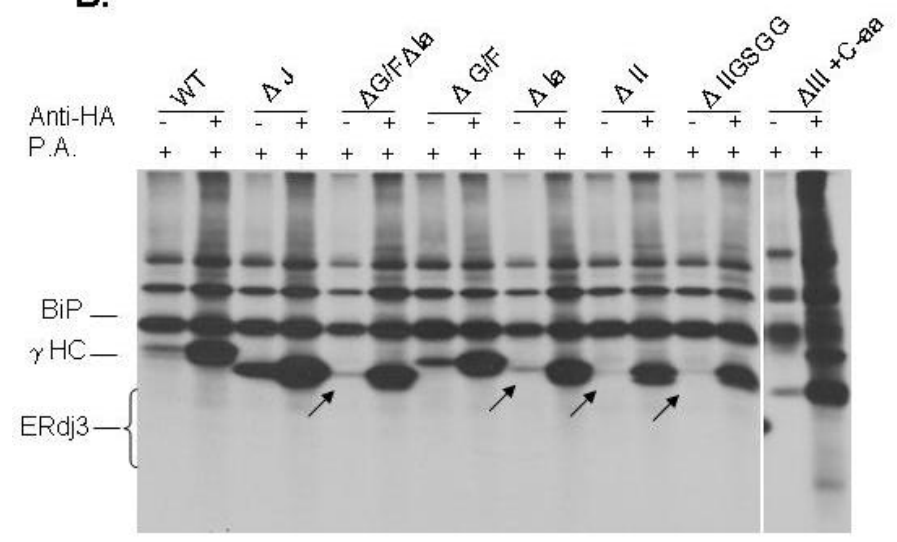

C.

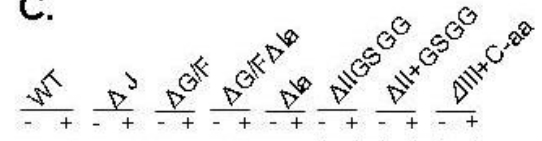

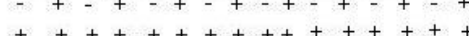

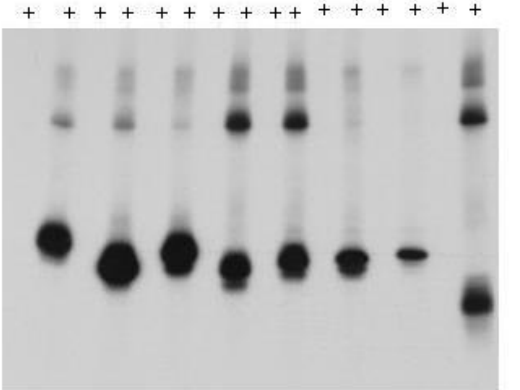

Figure 2-2. Determination of the ability of wild-type and mutant ERdj3 to bind $\gamma \mathrm{HC}$ in vivo

(A). A schematic drawing of the domain structure of the various mutants. In the case of one of the domain II mutants, a GSGG linker was added between domain Ia and Ib to aid in keeping the protein structure intact. (B). COS cells were cotransfected with $\gamma \mathrm{HC}$ and either WT ERdj3 or the indicated ERdj3 mutants and metabolically labeled proteins were immunoprecipitated as indicated. Samples were analyzed by reducing SDS-PAGE. (C). COS cells were transfected with each of the ERdj3 constructs alone and analyzed as in (B). 
mutant to $\gamma \mathrm{HC}$, which is consistent with our previous finding that mutation of the HPD motif in this domain resulted in better binding of mutant ERdj3 with several different substrate proteins in cells (Shen et al., 2005). Deletion of the Gly/Phe-rich region $(\Delta \mathrm{G} / \mathrm{F})$ did not obviously affect binding to $\gamma \mathrm{HC}$, and removing domain III only had a very modest effect on binding. When domain Ia was deleted alone or along with the Gly/Pherich region, we observed a slight decrease in the ability of the mutant proteins to associate with the substrate. Structural data on Ydj1 revealed that domain I is in direct contact with a peptide that was co-crystallized with $\mathrm{Ydj} 1$ and therefore is very likely to form at least part of the substrate binding site (Li et al., 2005). Domain I of Sis 1 also contains a similar substrate binding structure based on crystallographic data (Sha et al., 2000; Li et al., 2003; Li et al., 2006). Somewhat unexpectedly, we found that deletion of domain II $(\Delta \mathrm{II})$, which bears little homology to the corresponding domain in Ydj1 and is missing in Sis1, had the most dramatic effect on $\gamma \mathrm{HC}$ binding.

\subsubsection{Binding of ERdj3 to purified denatured luciferase in vitro}

A caveat to these data is that binding of even wild-type ERdj3 to $\gamma \mathrm{HC}$ in cells can only be detected with a chemical cross-linker due to the detergent sensitive nature of its association with substrates (Meunier et al., 2002), and the effects of most of the deletions are quite modest. Using PyMol (DeLano Scientific LLC) to model ERdj3 structure, we identified the residues that could potentially form the substrate binding patch in domain I of ERdj3. The model indicates that these hydrophobic residues are highly conserved between ERdj3 and Ydj1, suggesting that ERdj3 forms same substrate binding motif as Ydj1 (Figure 2-3A). Since we recently demonstrated that ERdj3 could bind to chemically denatured luciferase in solution and in a modified ELISA assay (Jin et al., submitted), we used this assay to further probe the regions of ERdj 3 required for substrate binding. Similar to data obtained for the in vivo binding of the various mutants to $\gamma \mathrm{HC}$, we found that deletion either domain Ia or II significantly diminished ERdj3's ability to bind to D-Luc (Figure 2-3B). According to structural data for Ydj1 and Sis1, domain I contains a hydrophobic pocket that forms the substrate binding site (Sha et al., 2000; Li et al., 2003). Deletion of domain Ia is likely to distort the overall structure of this domain, since it cooperates with domain Ib to form the intact domain I. Thus data obtained with this mutant cannot be clearly interpreted. When we aligned the structural model of ERdj3 with Ydj1, we found that four out of five amino acids in Ydj1's domain I that form the hydrophobic pocket were conserved in ERdj3 (Figure 2-3C). To examine the roles of these amino acids in ERdj3's ability to bind substrate, we mutated each of these four amino acids alone (I113A, V1332A, L226A, F241A), the first two amino acids together (I113A-V132A), or all of them together (IVLF-A) to alanine and tested their ability to bind to D-Luc in vitro. We found that except for I113A, all of the point mutations or combinations of them affected ERdj3's ability to bind to D-Luc (Figure 2-3C). This suggested that these hydrophobic amino acids are likely to also form the substrate binding site in ERdj3, which agrees with the structure model (Figure 2-3A). 
A.

Grey: substrate peptide

Green: Ydj1

Pink: ERdj3 model

Red: residues on Ydj1

Blue: residues on ERdj3
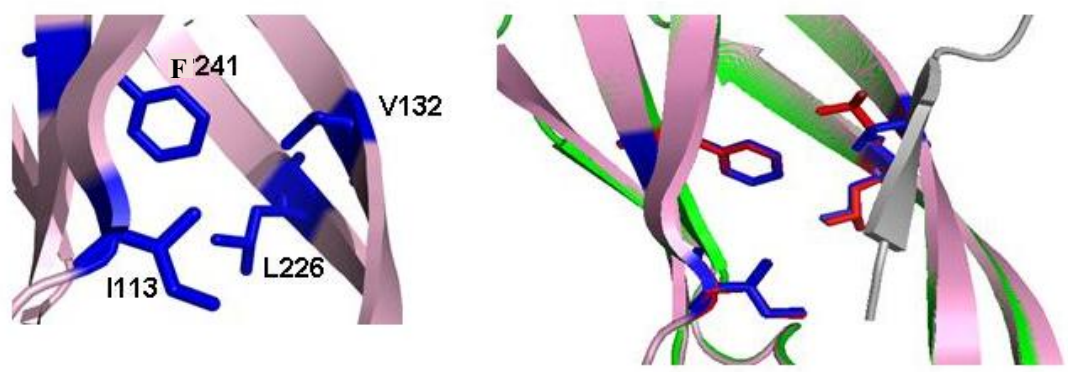

B.

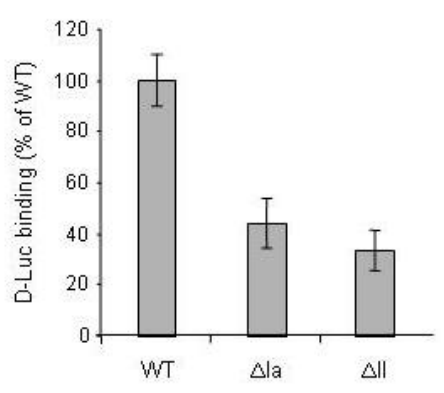

C. Point mutants in domain I affect substrate binding

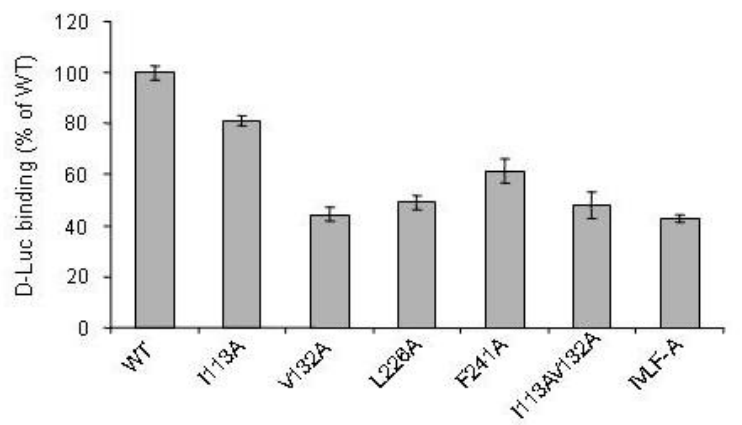

Figure 2-3. Both domain I and domain II of ERdj3 contribute to its ability to bind to D-Luc

(A) Ribbon diagram of the modeled structure of domain I of ERdj3 (left) showing amino acid residues that could potentially form the hydrophobic substrate binding patch described for Ydj1. An overlap of the substrates binding motifs of ERdj3 and Ydj1(right). Grey: substrate peptide; Green: Ydj1; Pink: ERdj3; Red: residues on Ydj1 that interacted with substrate; Blue: corresponding residues on ERdj3. (B) Measurement of complex formation between wild type and mutant ERdj3 and denatured luciferase.

The quantity of luciferase bound to WT ERdj3 was set as $100 \%$ of control, and the values for the mutants were expressed as a percentage of wild type ERdj3. (C) Measurement of complex formation between wild type ERdj3 and point mutants and denatured luciferase. Relative binding was determined as in (B). 


\subsubsection{ERdj3 forms dimer, which is important for substrate binding}

The yeast cytoplasmic DnaJ homologues, Ydj1 and Sis1, form dimers via sequences located in their $\mathrm{C}$ terminus, which are essential for substrate binding (Sha $e t$ al., 2000; Wu et al., 2005). Since ERdj3 is similar to Ydj1 in overall structure, we tested whether ERdj 3 forms dimers in cells. First, we made a $3 \times \mathrm{HA}-\mathrm{ERdj} 3$ construct, which contains three HA tags at the $\mathrm{C}$ terminus of ERdj3 in order to clearly separate it from the untagged versions of ERdj3. Each of tagged and untagged constructs were first expressed in COS cells alone. Cell lysates were prepared and immunoprecipitated with Protein A Sepharose beads alone, the anti-ERdj3 serum, or a monoclonal anti-HA antibody. The monoclonal anti-HA antibody interacted only with HA-tagged form of ERdj3, whereas the polyclonal anti-ERdj3 recognized both $3 \times$ HA-ERdj3 and ERdj3 (Figure 2-4A). The 3X HA-tagged form of ERdj3 was readily distinguished from ERdj3 on $10 \%$ SDS-PAGE gels. Next, the $3 \times$ HA-tagged form of ERdj 3 was co-expressed with untagged wild-type ERdj3. We observed that the anti-HA antibody precipitated both $3 \times$ HA-ERdj3 and ERdj3 at an approximately 1:1 ratio when they were co-expressed (Figure 2-4B lane 6 ), suggesting that $3 \times$ HA-ERdj3 and ERdj3 are associated with each other in cells as either dimers or multimers. Unlike ERdj3's association with substrate, ERdj3 dimers are stable in the presence of detergents and can be detected in the absence of crosslinker.

It has been reported that tyrosine (Y) 336 on Sis1 plays an important role in dimerization (Sha et al., 2000). Phenylalanine (F) 335 of Ydj1 is the counterpart of Y336 of Sis1 and is also critical for Ydj1 dimerization (Li et al., 2005; Wu et al., 2005). Sequence alignment of Sis1, Ydj1 and ERdj3 identified F326 on ERdj3 as the likely counterpart of F335 of Ydj1 and Y336 of Sis1. Thus, we mutated F326 to either alanine (A) or aspartic (D) amino acid on both an untagged or HA-tagged background. The indicated mutants were first expressed in COS cells and immunoprecipitated with either Protein A Sepharose beads alone, the anti-ERdj3 serum, or a monoclonal anti-HA antibody. All of the proteins were expressed and migrated to the expected position on the SDS-gels. HA-tagged ERdj3 constructs were recognized by both anti-ERdj3 and antiHA antibody, whereas the untagged forms were only precipitated by anti-ERdj3 antibody. None of them bound to Protein A Sepharose beads nonspecifically. To examine whether ERdj3 F3326A/D form dimers with wild type ERdj3, HA-tagged wild type ERdj3 was co-expressed with the untagged F326A or F326D ERdj3 mutants and immunoprecipitation experiments were performed (Figure 2-4B lane 7-12). We found that untagged F326A/D was not co-precipitated along with HA-tagged wild type ERdj3 when it was precipitated with the anti-HA antibody, demonstrating that the F326A/D mutations affected the ability of ERdj3 to form dimers. We also co-expressed untagged F326A/D ERdj3 along with HA-tagged F326A/D ERdj3 (Figure 2-4B lane 13-18). Again, we found that the mutation of F326 to either alanine or aspartic acid abolished ERdj3's ability to forms dimers.

The ability of F326D ERdj3 to bind to denatured luciferase was next examined. We found that this mutation decreased the binding of ERdj3 to D-Luc, as did deletion of domain III $(\Delta \mathrm{III})$, which lacks the dimerization residue (Figure 2-5). The fact that dimerization mutants (F326A/D and $\Delta \mathrm{III})$ diminished the ability of ERdj3 to bind to 
A.

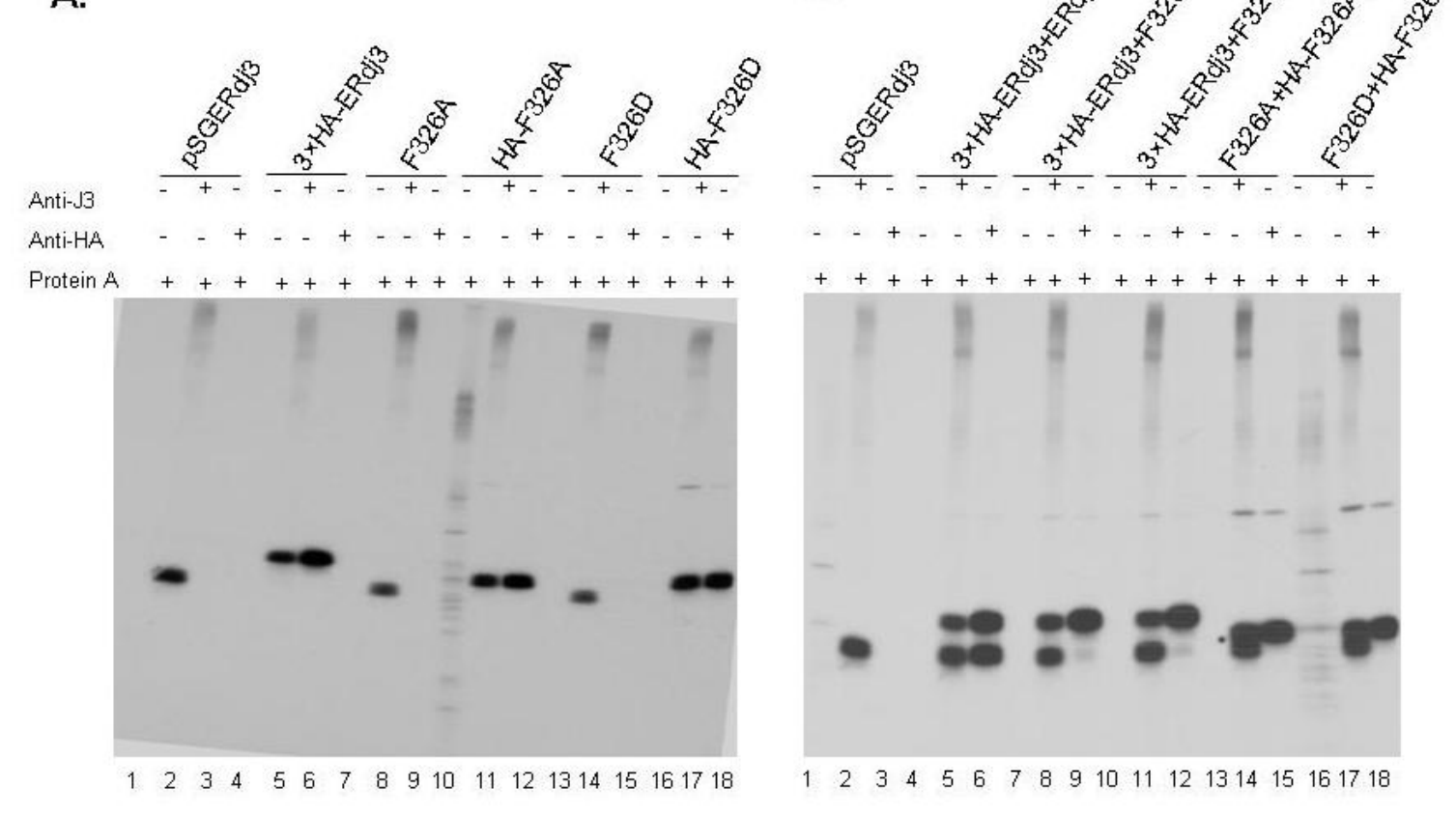

\section{Figure 2-4. ERdj3 forms dimers in cells}

COS cells were transfected with cDNA encoding pSG-ERdj3 (lane1-3), 3×HA-ERdj3 (lane 4-6) (with HA epitope tagged at its C-terminus), ERdj3 F326A (lane 7-9), F326D (lane 13-15), HA-ERdj3 F326A (lane10-12) and HA-ERdj3 F326D (lane 16-18) either alone (A) or co-transfected with $3 \times$ HA-ERdj3 (B). Metabolically labeled cell lysates were divided into three equal parts and incubated with indicated antibodies. Samples were analyzed by reducing SDS-PAGE. 


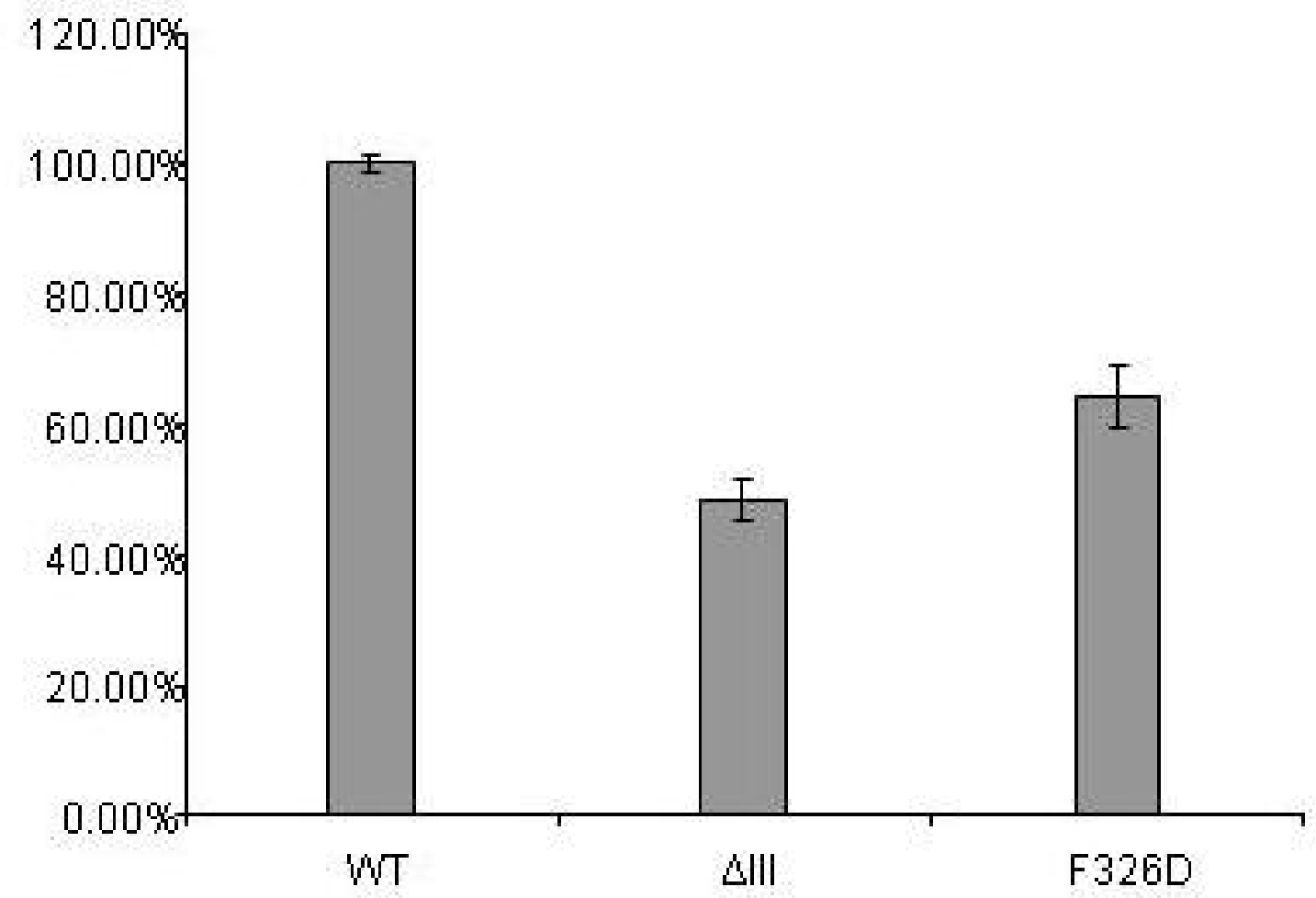

Figure 2-5. Deletion of domain III or mutation of Phe326 to aspartic acid affects ERdj3's substrate binding ability.

ELISA assay for luciferase binding was performed and quantitated as described in Figure 2-3B. 
substrate strongly argues that dimerization is important for ERdj3's substrate binding, which is consistent with what has been reported for Ydj1 and Sis1 (Wu et al., 2005).

\subsection{Discussion}

According to their domain conservation with E. coli DnaJ, DnaJ proteins have been divided into three subgroups. Type I and II DnaJ proteins appear to bind directly and promiscuously to the unfolded regions of multiple substrates through their substrate binding domain, whereas type III proteins either do not bind to unfolded proteins or have a very limited client repertoire. A crystal structure has been obtained for the $\mathrm{C}$ terminal fragment of Ydj1 (102-384 aa) with a bound peptide (GWLYEIS) (Li et al., 2003). This fragment lacks both the $\mathrm{N}$ terminal $\mathrm{J}$ domain and $\mathrm{G} / \mathrm{F}$ rich flexible linker and contains a mutation of phenylalanine 335 to aspartic acid, which disrupts its ability to form dimers (Li et al., 2003). The structure revealed that the peptide bound to a hydrophobic pocket on domain I and made contact with five different polar residues in this pocket. The importance of these residues in binding to peptide was confirmed with mutagenesis studies (Li et al., 2005). The structure of the corresponding $\mathrm{C}$ terminal region of Sis 1 had been solved previously, but in this case there was no substrate bound (Sha et al., 2000). Although the amino acid sequence homology is not high between these two proteins in this region, their tertiary structures were nearly identical, except that Sis1 lacks the Cysrich domain II (Li et al., 2003), suggesting that domain I of Sis1 is likely to contain the substrate binding site. This possibility was supported by mutagenesis studies in which mutations in the hydrophobic pocket affected Sis1's ability to bind substrate (Lee et al., 2002). Our data revealed that ERdj3 should be considered to be a type I DnaJ protein even though its domain II is quite different from that of Ydj1. We found that most of the hydrophobic amino acids that form the substrate binding site on Ydj1 are conserved in ERdj3. Mutations of these amino acids alone or in combination affected ERdj3's ability to bind to D-Luc in vitro. Overall these studies suggest that this substrate binding structure may be common to all type I and II DnaJ proteins. This possibility is further supported by earlier peptide binding studies on DnaJ (Rudiger et al., 2001), where it was found that DnaJ preferred to bind peptides with hydrophobic features.

Type I and II DnaJ proteins form homodimers, and the dimerization motif is located in the $\mathrm{C}$ terminus of these proteins (domains III and II respectively). Both Ydj1 and Sis 1 dimerize via a number of hydrophobic residues that form a hydrophobic patch. Mutation of F335 of Ydj1 to aspartic acid disrupted the hydrophobic patch and yielded monomers (Li et al., 2005). In the case of Ydj1, residues in $\beta$-strands in domain III of one monomer can interact with those of another monomer to contribute to homodimer formation (Wu et al., 2005). Disruption of dimer formation for both Ydj1 and Sis1 results in severe defects in their ability to bind to substrates and to facilitate Hsp70's ability to refold substrates (Wu et al., 2005). We demonstrated that ERdj3 forms dimers or multimers in cells. Our in vivo study revealed that the F326A/D mutation abolished ERdj3's ability to form dimers. Similar to data obtained with Ydj1, this affected ERdj3's ability to interact with substrates. This finding supports a model where the dimerization of both type I and II DnaJ proteins allows the two substrate binding domains to form a clamp around unfolded substrates (Landry, 2003). Although type III DnaJ proteins are 
much less conserved, some of them have been shown to have substrate proteins (Gruschus et al., 2004). Although it has not been examined, it is possible that this group of proteins does form dimers or conversely that dimerization is more important for the ability to interact with a broader range of substrates, which is a hallmark of the type I and II DnaJ proteins.

Our in vivo assay to examine the binding of ERdj3 mutants with $\mathrm{HC}$ revealed that the $\triangle$ III ERdj3 mutant, which lacked the dimerization domain, bound to $\mathrm{HC}$ as well as WT ERdj3, whereas in the in vitro assay, the association of the $\Delta$ III ERdj3 with D-Luc was significantly diminished. We can think of four possibilities to account for this, which are not mutually exclusive. First, in the in vivo assays, a crosslinker was used, which could serve to stabilize transient interactions between ERdj3 and the substrate. Second, since a number of chaperones form a complex in the ER of cells, which includes $\mathrm{BiP}$ (Meunier et al., 2002), it is possible that the substrate is binding to one of these and not to the $\triangle$ III ERdj3 mutant directly. Third, although deletion of domain III or mutation of F326 to alanine or aspartic acid disrupted dimer formation, the use of crosslinking agents revealed that some dimers could be still be detected with these mutants (data not shown). Thus, it is conceivable that the use of crosslinker might stabilize the interaction between the $\Delta$ III mutant and endogenous ERdj3, which would result in the apparent association of $\triangle \mathrm{III}$ with $\mathrm{HC}$ in vivo. Finally, it is possible that the differences we are observing in the in vivo versus in vitro assay are actually reflecting distinct affinities of ERdj3 for these two substrates.

Our results argue that domain II is critical for ERdj3's substrate binding ability. This Cys-rich region in other type I proteins contributes to their chaperone activities by affecting the transfer of substrate to the Hsp70 partner (Lu et al., 1998). Previously we reported that ERdj3 contains intra-chain disulfide binds (Shen et al., 2005). Only four cysteines are found in ERdj3, and they are all in domain II, arguing that they are responsible for the intra-chain disulfides and do not form a zinc-finger binding site as in other type I DnaJ proteins, which was confirmed by recent studies (Marcus et al., 2007). Although domain II of ERdj3 is very different from that of Ydj1, it is very highly conserved among ERdj3 proteins in different species (about $80 \%$ identity between human and C. elegans). However, Sis1, which possesses a very a similar overall substrate binding structure as $\mathrm{Ydj} 1$, does not possess a domain II. Of interest, the structure of the Ydj1 C-terminal fragment could only be obtained with a bound peptide, whereas Sis 1's was crystallized without the presence of a peptide. Considering these facts, we propose that domain II could play a role in stabilizing domain I of type I DnaJ proteins in the absence of substrate. Alternatively is it possible that the substrate binding repertoire of type I proteins is different than that of type II DnaJ proteins and therefore requires a more complex binding site. But the fact that both Ydj1 and Sis1 binds to denatured luciferase in vitro argues against this possibility.

In summary, we have demonstrated that domain I and II of ERdj3 contribute to substrate binding. The hydrophobic residues in domain I of ERdj3 apparently form a substrate binding site that resembles that of Ydj1. The role of domain II is less clear but could be important in maintaining domain I in a configuration that is critical for substrate 
binding. In addition we found that ERdj3 exists as a dimer in cells, which contributes to substrate binding. Together these data help to define the structural elements required for substrate binding for DnaJ proteins. 


\section{CHAPTER 3. FUNCTIONAL CONSERVATION BETWEEN ERDJ3 AND YDJ1, DNAJ HOMOLOGUES IN THE MAMMALIAN ER AND YEAST CYTOPLASM}

\subsection{Introduction}

Hsp70 proteins constitute a highly conserved group of molecular chaperones that are found in all organisms and all cellular organelles. They play critical roles in most cellular functions due to their ability to bind to unfolded regions on nascent proteins or subunits of heteromeric complexes in a nucleotide-dependent manner (Munro \& Pelham, 1986; Liberek et al., 1991; Buchberger et al., 1995). The ATPase activity of Hsp70 proteins is tightly regulated by DnaJ proteins that increase the hydrolysis of ATP leading to enhanced binding of the Hsp70s to substrates (Liberek et al., 1991) and nucleotide exchange factors that release them (Liberek et al., 1991; Chung et al., 2002). Some DnaJ proteins bind directly to unfolded regions on substrate proteins through their substrate binding domain and serve to recruit the ATP-bound form of their Hsp70 partner to the unfolded substrate (Rudiger et al., 2001). The number of DnaJ proteins in most organisms/organelles far exceeds that of Hsp70s. A single Hsp70 can interact with multiple DnaJ proteins and form unique DnaJ/Hsp70 pairs (Caplan et al., 1993; Cheetham \& Caplan, 1998; Sahi \& Craig, 2007). This has led to the hypothesis that different DnaJ proteins selectively interact with their Hsp70 partner and regulate its specific functions.

DnaJ proteins regulate the ATPase activity of their Hsp70 partner via direct interaction with the nucleotide binding domain, which occurs through a highly conserved $\sim 70$ amino acid J domain (Wall et al., 1994). J domains contain a signature tri-peptide HPD (Histidine-Phenylalanine-Aspartic acid) motif, which is critical for inducing Hsp70s' ATPase activity (Wall et al., 1994; Tsai \& Douglas, 1996). In many cases DnaJ proteins are not interchangeable between organelles or organisms even though the $J$ domains are highly conserved. This suggests that there is some specificity between DnaJ/Hsp70 pairs; at the level of substrate recognition, the ability to functionally interact with the particular Hsp70, or the ability to fold appropriately within a given organelle. A recent genetic study was conducted to test the ability of 13 different yeast cytosolic DnaJ proteins to functionally interact with resident Hsp70s (Sahi \& Craig, 2007). It was found that in many cases the ability to stimulate the ATPase activity of a particular Hsp70 was sufficient to constitute a functional pair. However, specific features of DnaJ proteins, such as the ability to interact with substrates, can also be required (Johnson \& Craig, 2001).

ERdj3 is a mammalian ER type I DnaJ family member, which binds to unfolded substrates (Yu et al., 2000; Meunier et al., 2002; Shen et al., 2005). The structural features of ERdj3 that contribute to substrate binding were found to closely resemble those of Ydj1 (Chapter 2). In addition to identifying the hydrophobic residues in domain I that are likely to form the substrate binding site, it was found that ERdj3 dimerizes, which significantly enhances substrate binding. Somewhat unexpectedly, it was ascertained that domain II of ERdj3, which is significantly different from the domain II in 
other type I DnaJ proteins, including Ydj1, was most important for substrate binding. The identification of critical features for substrate binding encouraged us to attempt to establish their roles in vivo using yeast complementation studies and to probe the requirements for establishing functional Hsp70/DnaJ pairs.

We found that Ydj1 bound to BiP and ERdj3 substrates when it was expressed in the mammalian ER. The reciprocal experiment was employed to determine whether ERdj3 could be functional in yeast. A cytosolically expressed, ER membrane tethered form of ERdj3 rescued the slow growth phenotype of the hlj1 1 ydj 1-151 mutant strain, while the ERdj3 mutations that affected substrate binding did not. Perhaps most surprisingly, deletion of domain II also inhibited complementation even though domain II of ERdj3 is much smaller and has a very different sequence than that of domain II of Ydj1. Expression of ERdj3 in the yeast ER did not rescue the growth defect of yeast containing mutations in two lumenal DnaJ homologs, SCJI and JEMI. This was likely due to the inability of ERdj3 to stimulate the ATPase activity of yeast BiP/ Kar2p, the ER lumenal $\mathrm{Hsp} 70$. Our studies provide further insights into requirements for producing functional Hsp70/DnaJ pairs and argue that in addition to requirements for functional interactions between these two proteins, the substrate binding properties of DnaJ proteins might be critical to specify the in vivo function of chaperone pairs.

\subsection{Materials and Methods}

\subsubsection{Preparation of Ydj1 and ERdj3 constructs}

To express Ydj1 in the mammalian ER, PCR was used to engineer an ER-targeting signal-sequence onto the N-terminus of Ydj1 using pBS-Ydj1 as a template. Primer pairs include:

5' primer: CGGGATCCatggctccgcagaacetgagcacettttgcctgttgctgctatacctcatcggg gcggtgattgccGTTAAAGAAACTAAGTTTTACGATATTCTAGGTGTTCC and 3' primer: CGGGATCCTCATTGAGATGCACATTGAACACCTTC.

The lower case letters represent the inserted ER targeting signal sequence and the italics indicate a BamHI site. The PCR product was digested with BamHI and inserted into 3HA-DSL mammalian expression vector. The translation stop codon in the 3HA-DSLssYdj1 construct was destroyed using the Quick change site-directed PCR kit (Stratagene) with the following primers.

5' primer: GAAGGTGTTCAATGTGCATCTCAAGGATCCCCGGAATTCCTCGAG 3' primer: CTCGAGGAATTCCGGGGATCCTTGAGATGCACATTGAACACCTTC The resulting PCR product has an HA tag at the C-terminus, which was already present in the 3HA-DSL vector. A Kozak sequence (underlined) was inserted at the translation start site of 3HA-DSL- ssYdj1 using Quick change PCR kit with the following primers: 5'primer:GTTTAAACGGATCCACCCGGGACAGAGGAACCATGGCTCCGCAGAA $\mathrm{C}$ and 3'primer:GTTCTGCGGAGCCATGGTTCCTCTGTCCCGGGTGGATCCGTTTA $\mathrm{AAC}$

A construct that would produce a soluble luminal form of Ydj1 (ssYdj1 C406S) was generated by mutating the $\mathrm{C}$ terminal farnesylation site with the primer pair: 
5' primer: GGTGGCGAAGGTGTTCAAAGTGCATCTCAAGGATCCCCG and 3' primer: CGGGGATCCTTGAGATGCACTTTGAACACCTTCGCCACC

To express ERdj3 in yeast, two constructs were produced under the control of the GPD promoter in the multi-copy pGPD426 vector. Full length ERdj3 containing a signal sequence for luminal ER expression was made by PCR using 3HA-DSL-ERdj3 vector as the template with the following primer pair:

5' primer: CGGAATTCGGACCCGGGAC

3' primer: CGGGATCCATATCCTTGCAGTCCATTGTATACCTTCTG

The resulting PCR product was digested with EcoRI and BamHI and inserted into the pGPD426 yeast expression vector (pGPD-ERdj3). For cytosolic expression at the ER membrane, CaaX-ERdj3 was generated using the following primer pair with 3HA-DSLERdj3 serving as the template:

5' primer: CGGGATCCGGAACCATGGGACGAGATTTCTATAAGATCTTGGGG 3' primer: CCCAAGCTTTCATTGAGATGCACATTGCAGTCCATTGTATACCTTC TGC

The resulting PCR product lacked the N-terminal signal sequence, and the C-terminal GY was replaced with a "CaaSQ" farnesylation sequence. The PCR product was digested with BamHI and HindIII and subcloned into the pGDP vector (pGDP-CAAX-ERdj3) to allow it to be expressed in yeast. All mutants were generated by QuickChange PCR with the primer pairs previously described (Chapter 2).

\subsubsection{Expression and detection of Ydj1 in mammalian cells}

Cells were transfected with the indicated vectors using the FuGENE 6 transfection reagent (Roche). After 48 hours, cells were labeled with ${ }^{35} \mathrm{~S}$ Translabel (Amersham Biosciences) for $3 \mathrm{~h}$, and cell lysates were prepared as described previously (Jin et al., submitted). To stabilize complexes between the various DnaJ-like proteins and either

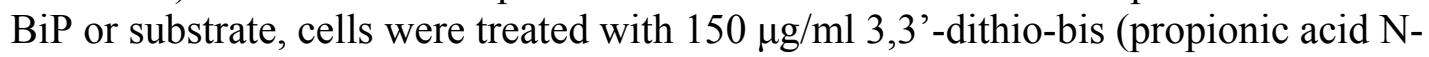
hydroxysuccinimide ester: DSP) for $1 \mathrm{~h}$ on ice and lyzed in NP40 lysing buffer after quenching with $100 \mu \mathrm{l}$ of $1 \mathrm{M}$ glycine. Solubilized proteins were incubated with the indicated antisera followed by precipitation with Protein A Sepharose beads. The immunoprecipitated complexes were subjected to SDS-PAGE and analyzed under reducing conditions. The signal was enhanced using Amplify (Amersham Bioscience) and detected by autoradiography.

\subsubsection{Protein expression, purification and ATPase assay}

His-tagged recombinant murine ERdj3 (wild type and mutants) and hamster BiP proteins were expressed in E. coli M15 cells and purified under non-denaturing conditions using $\mathrm{Ni}^{2+}$-agarose (Qiagen QIAexpress System) as described (Jin et al., submitted). Briefly, ERdj3 proteins were induced with $0.1 \mathrm{mM}$ isopropyl $\beta$-Dthiogalactoside (Sigma) followed by growth for $18 \mathrm{~h}$ at $18^{\circ} \mathrm{C}$, whereas the BiP protein was induced with $1 \mathrm{mM}$ IPTG at $37^{\circ} \mathrm{C}$ for $2 \mathrm{hrs}$. ERdj3 recombinant proteins were stored at $-20^{\circ} \mathrm{C}$ in $25 \mathrm{mM}$ sodium phosphate buffer containing $150 \mathrm{mM} \mathrm{NaCl}, 0.02 \%$ Triton $\mathrm{X}$ - 
100, 50\% glycerol and Complete EDTA-Free Protease inhibitor cocktail tablet (Roche). BiP protein was stored in $20 \mathrm{mM}$ HEPES buffer ( $\mathrm{pH} 7.2$ ) with $50 \mathrm{mM} \mathrm{KCl,} 5 \mathrm{mM} \mathrm{MgCl} 2$, $0.01 \%$ NP-40, 50\% glycerol, and Complete EDTA-Free Protease inhibitor cocktail tablet.

\subsubsection{ATPase assays}

The following proteins were purified using previously established protocols: Ssa1p (McClellan et al., 1998), Ydj1p (Cyr et al., 1992), GST-tagged J-domain of Hlj1p (Youker et al., 2004), hexahistidine-tagged yeast BiP/Kar2p (McClellan et al., 1998), and the GST-tagged J-domain of Sec63p (Corsi \& Schekman, 1997). Steady-state ATPase assays using the indicated molar ratios of DnaJ protein to Hsp70 were performed as described (Cyr et al., 1992; McClellan et al., 1998).

\subsubsection{Rescue of the slow growth phenotype of the Ydj1 mutant strain}

The following Saccharomyces cerevisiae yeast strains were used for complementation studies: HLJ1 YDJ1 (MAT $\alpha$ ade2-1 leu2-3,112 his3-11,15 trp1-1 ura31 can1-100), hlj1 1 ydj1-151 (MAT $\alpha$ ade2-1 leu2-3,112 his3-11,15 trp1-1 ura3-1 can1100 hlj1 $1:: T R P 1$ ydj1-2::HIS3 LEU2::ydj1-151) (Youker et al., 2004), SCJ1 JEM1

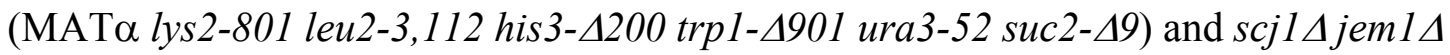
(MAT $\alpha$ ade2-1 leu2-3,112 his3-11,15 trp1-1 ura3-1 can1-100 scj14::TRP1 jem1 $1:: L E U 2)$ (Nishikawa \& Endo, 1997). The yeast strains were grown to logarithmic phase at $26^{\circ} \mathrm{C}$ in yeast extract-peptone-dextrose medium containing $2 \%$ glucose and transformed with the indicated plasmids using lithium acetate (Ito et al., 1983). The resulting transformants were selected and grown to logarithmic phase at $26^{\circ} \mathrm{C}$ in synthetic complete medium containing $2 \%$ glucose. 10 -fold serial dilutions were spotted onto solid medium and cultured at the indicated temperatures for 2 days.

\subsection{Results}

\subsubsection{Ydj1 expressed in the mammalian ER binds to BiP and ERdj3 substrates}

We found that the mammalian ER localized DnaJ orthologue, ERdj3, appears to be structurally very similar to Ydj1, a yeast cytosolic DnaJ protein (Chapter 2) with the exception of domain II, which was demonstrated to be important for substrate binding (Figure A-1). To better understand restrictions guiding Hsp70/DnaJ pairs, mammalian $\mathrm{BiP}$ was co-expressed in COS cells with a C-terminal HA-tagged ERdj3 or two different Ydj1 constructs engineered with an $\mathrm{N}$-terminal ER signal-sequence and a C-terminal HA tag. In one construct, Ydj1's farnesylation site was removed (ssYdj1C406S) and the other retained it (ssYdj1). Both can be expressed in mammalian ER (Figure A-2). To determine their ability to associate with BiP, ssYdj1 or ssYdj1 C406S and BiP were coexpressed in COS cells. Forty-eight hours post-transfection, cells were metabolically labeled and treated with DSP to stabilize BiP: DnaJ protein interactions (Meunier et al., 
2002). ERdj3 and BiP were co-expressed as a positive control for association. Coimmunoprecipitation experiments revealed that both Ydj1 proteins could associate with BiP as well as ERdj3 did (Figure 3-1A), regardless of whether the complex was detected with an anti-BiP or an anti-HA monoclonal antibody that recognized the epitope tag on the DnaJ proteins. Neither of the Ydj1 proteins bound nonspecifically to Protein-A Sepharose beads.

The ability of Ydj1 to bind to an immunoglobulin $\kappa$ light chain ( $\kappa L C)$, which is a substrate of both BiP and ERdj3 (Shen et al., 2005) was examined. Cells were cotransfected with constructs encoding $\kappa L C$ along with either ERdj3 or ssYdj1. It was found that ssYdj1 associated with $\kappa L C$ as efficiently as ERdj3 (Figure 3-1B). The fact that an ER expressed form of Ydj1 could interact with both BiP and $\kappa L C$ suggested that Ydj1 was likely to be functional in the ER of mammalian cells. This led to the possibility that ERdj3 might be functional in yeast.

\subsubsection{Overexpression of ERdj3 does not rescue the temperature-sensitive defect of the Ascj14jem1 strain}

Because ERdj3 is an ER localized protein that contains intra-chain disulfide bonds, its ability to function in the yeast ER was tested first. Scj1 and Jem1 are two yeast ER DnaJ proteins that interact with Kar2p, the yeast ER Hsp70 (Schlenstedt et al., 1995). They are required for multiple ER functions including protein folding in the yeast ER and ER associated degradation (ERAD) (Nishikawa et al., 2001). Because Scj1 has a similar apparent molecular weight as ERdj3 and is also a soluble protein, it has been suggested to be the homolog of ERdj3 (Figure A-1). Loss of both Scj1 and Jem1 (4scj14jem 1) induces the unfolded protein response (Nishikawa and Endo, 1997; Silberstein et al., 1998 ) and reduces ERAD efficiency for soluble ERAD substrates. Growth of the $\triangle s c j 14 j e m 1$ strain is unaffected at $26^{\circ} \mathrm{C}$ but exhibits a slow growth phenotype at elevated temperatures (Nishikawa \& Endo, 1997). An ERdj3 construct was created that could be expressed under the control of the GPD promoter in the multi-copy pGPD426 vector. Wild type ERdj3 contains an ER signal sequence and thus is targeted to the ER, while the CaaX-ERdj3 mutant no longer contains the ER targeting signal sequence and has a CaaSQ farnesylation sequence at the C-terminus, targeting it to the cytosolic side of the ER membrane. The farnesylation sequence is the same as that found in Ydj1 and is necessary for Ydj1's function at elevated temperatures (Caplan et al., 1992). Expression of ERdj3 did not affect the growth of wild-type Scj1Jeml yeast cells (Figure 3-2A). However, ER expressed ERdj3 was unable to rescue the slow growth phenotype of the $\Delta S c j 1 \Delta$ Jem 1 strain at elevated temperatures (Figure 3-2A). This was not due to poor expression of mammalian ERdj 3 in the yeast ER as determined by fluorescence staining (data not shown). As a major function of DnaJ proteins is to stimulate the ATPase activity of their Hsp70, we examined the ability of ERdj3 to stimulate the ATPase activity of Kar2p. As expected, the basal ATPase activity of Kar2p was quite low but could be stimulated $\sim 4.3$ fold by the J domain of Sec63, a yeast ER DnaJ (Corsi \& Schekman, 1997). However, ERdj3 only modestly enhanced the ATPase activity of Kar2p (about 1.3 fold), suggesting that this might be the reason that ERdj3 could not complement the $\Delta S c j 1 \Delta J e m 1$ strain. (Figure 3-2B) 
A.

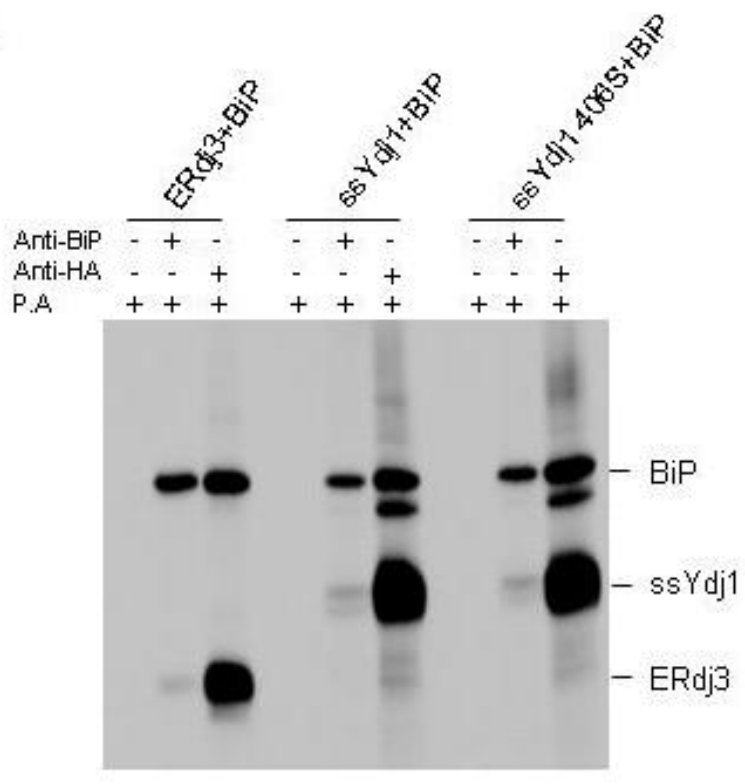

B.

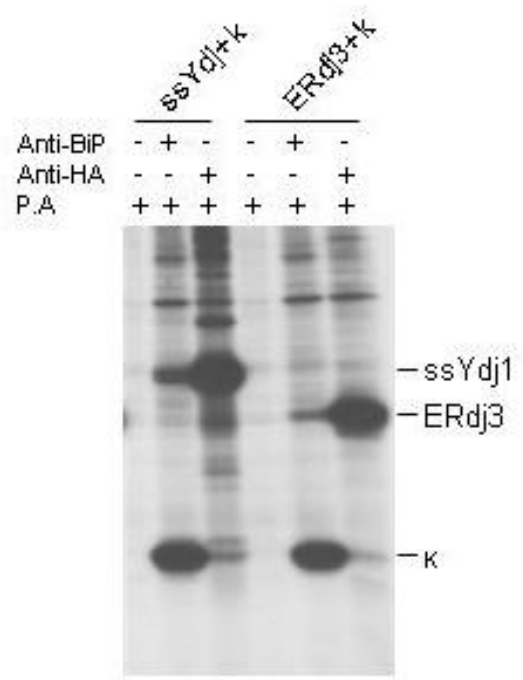

Figure 3-1. Mammalian ER expressed Ydj1 interacts with both BiP and kLC

(A) Cos cells were co-transfected with cDNAs encoding BiP and the indicated HAtagged ERdj3 or Ydj 1 constructs. Metabolically labeled, cross-linked cell lysates were immunoprecipitated with anti-HA, anti-BiP or Protein A Sepharose alone. Isolated proteins were separated by reducing SDS-PAGE. (B) Cos cells were co-transfected with cDNAs encoding $\kappa L C$ and either ERdj3 or Ydj1 constructs. Samples were analyzed as described in (A). 
A.

26

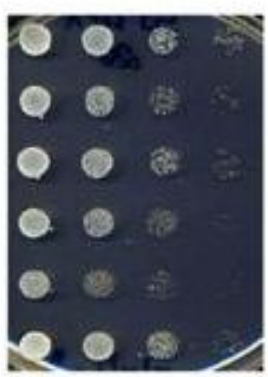

30

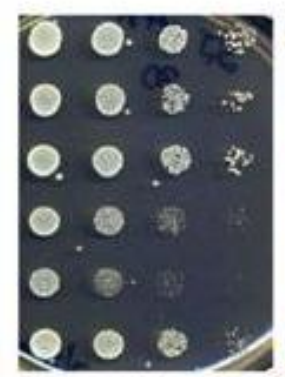

35

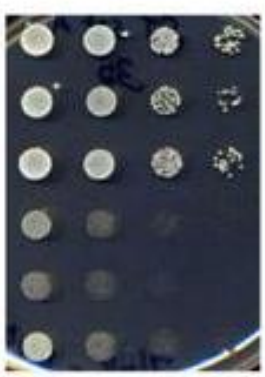

37

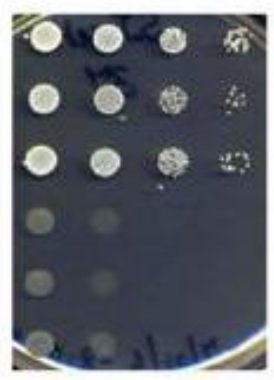

B.

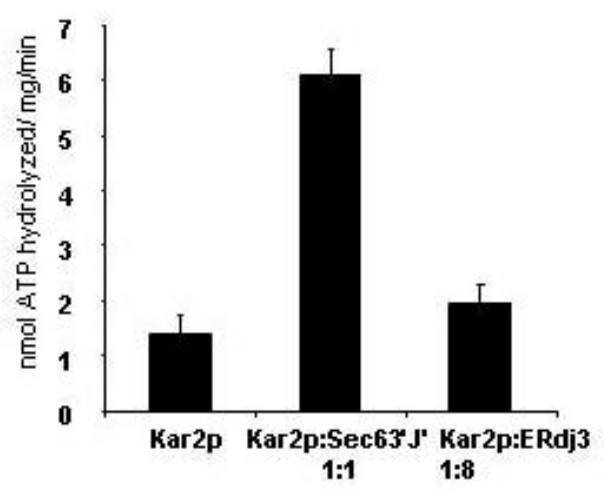

Figure 3-2. ER expressed ERdj3 does not rescue the slow growth phenotype of the $\Delta$ scj1 14jem 1 strain

(A) Ten-fold serial dilutions were performed on selective media of wild-type

(SCJ1JEM1) and mutant ( $\triangle$ scj1 1 jem 1 ) yeast strains containing an empty vector (GDP), a vector for the expression of an ER-targeted form (ERdj3), or a vector encoding a cytosolically localized form $(\mathrm{CaaX})$ of full-length ERdj3. Each protein was expressed from a multi-copy vector containing the constitutive $P G P D$ promoter. The plates were incubated for 2 days at the indicated temperatures. (B) ERdj3 does not stimulate the ATPase activity of Kar2p. The ATPase activity of Kar2p was measured either by itself or in the presence of either ERdj3 or the J domain of Sec63. Reactions contained $1 \mathrm{mg}$ of Kar2p, 1:1 molar ratio of Kar2p: Sec63 J domain, or an eight-fold molar excess of the ERdj3 protein. ATPase activity is expressed as nmoles of ATP hydrolyzed per milligram of protein per minute (nmol ATP hydrolyzed $/ \mathrm{mg} / \mathrm{min}$ ). Data represent the means of a minimum of three independent experiments. 


\subsubsection{Overexpression of cytosolically localized ERdj3 rescues the temperature-sensitive defect of the hlj14 ydj1-151 strain}

Since our previous data suggested that ERdj3 was structurally very similar to Ydj1, we next determined if it could compensate for the loss of Ydj1. Ydj1p and Hlj1p (two yeast cytosolic DnaJ proteins) interact with Ssa1p, the yeast cytosolic Hsp70 and are functionally redundant (Youker et al., 2004). The hlj1 $\Delta$ ydj1-151 mutant strain lacks Hlj1p and expresses a conditional mutant form of Ydj1, Ydj1-151p. This mutant shows reduced efficiency for ER-associated degradation (ERAD) and exhibits a slow growth phenotype at elevated temperatures (Youker et al., 2004). Expression of CaaX-ERdj3 in the cytosol of wild-type HLJ1 YDJ1 yeast cells did not affect their growth (Figure 3-3A). However, when the hlj1 $\Delta$ ydj1-151 strain expressing CaaX-ERdj3 was grown at elevated temperatures, we found that the expression of CaaX-ERdj3 allowed growth at temperatures up to $37^{\circ} \mathrm{C}$ (Figure 3-3A). Recombinant ERdj3 was tested for its ability to stimulate the ATPase activity of Ssal and found to do so at a level similar to Hlj1 (Figure 3-3B). These results demonstrated that CaaX-ERdj3 could act as a cochaperone of Ssalp and compensate for the loss the Ydj1, which allowed us to next examine the requirements for substrate binding by expressing the various ERdj3 mutants.

\subsubsection{Mutations in ERdj3 that affect substrate binding are unable to rescue the slow growth phenotype of hlj14 ydj1-151 strain}

The binding of ERdj3 to substrates was recently shown to be dependent on three things: 1) the presence of domain II, 2) the pocket formed by hydrophobic amino acids in domain I, and 3) dimerization, which occurs through the interactions in the C-terminal region of which phenylalanine 326 is critical (Chapter 1). To determine whether the ability to bind substrates is required for ERdj3 to compensate for the loss of Ydj1, seven ERdj3 mutants were expressed in yeast. These included ones in which domain II was either simply deleted $(\Delta \mathrm{II})$ or replaced by a "GSGG" linker sequence ( $\Delta \mathrm{II}-\mathrm{GSGG})$, two mutants in which dimerization was inhibited (F326A and F326D), and finally, two single amino acid substitutions in domain I (I134A and L208A) and one in which all four hydrophobic residues affecting substrate binding were altered (IVLF-A). All mutations were constructed on the CaaX-ERdj3 background. Expression of each of these mutants in the HLJI YDJI strain did not affect cell growth (Figure 3-4A), however, none of them were able to rescue the temperature-sensitive slow phenotype of the hlj1 $\Delta$ ydj1-151 strain (Figure 3-4B). Indeed, expression of most of the mutants actually enhanced the temperature-sensitivity of this strain (Figure 3-4B). The fact that the CaaX-F326A/D mutants were unable to rescue the slow growth phenotype, argues that dimerization of CaaX-ERdj 3 is required for its function in yeast. The failure of these mutants to complement was not due to an inability to stimulate the ATPase activity of Ssa1, since all mutants were able to stimulate ATP hydrolysis to levels that are comparable to both Hlj1 and wild-type ERdj3 (Figure 3-4C). Nor was it likely to be due to inadequate expression, as at least two of the mutants, $C A A X-\triangle \mathrm{II}-\mathrm{GSGG}$ and $C A A X-F 326 D$, were expressed at similar levels as the wild-type CaaX-ERdj3 protein (Figure A-3). These results argue that the cytosolically disposed ERdj3 requires its substrate binding activity to constitute a functional pair with Ssal in yeast. 


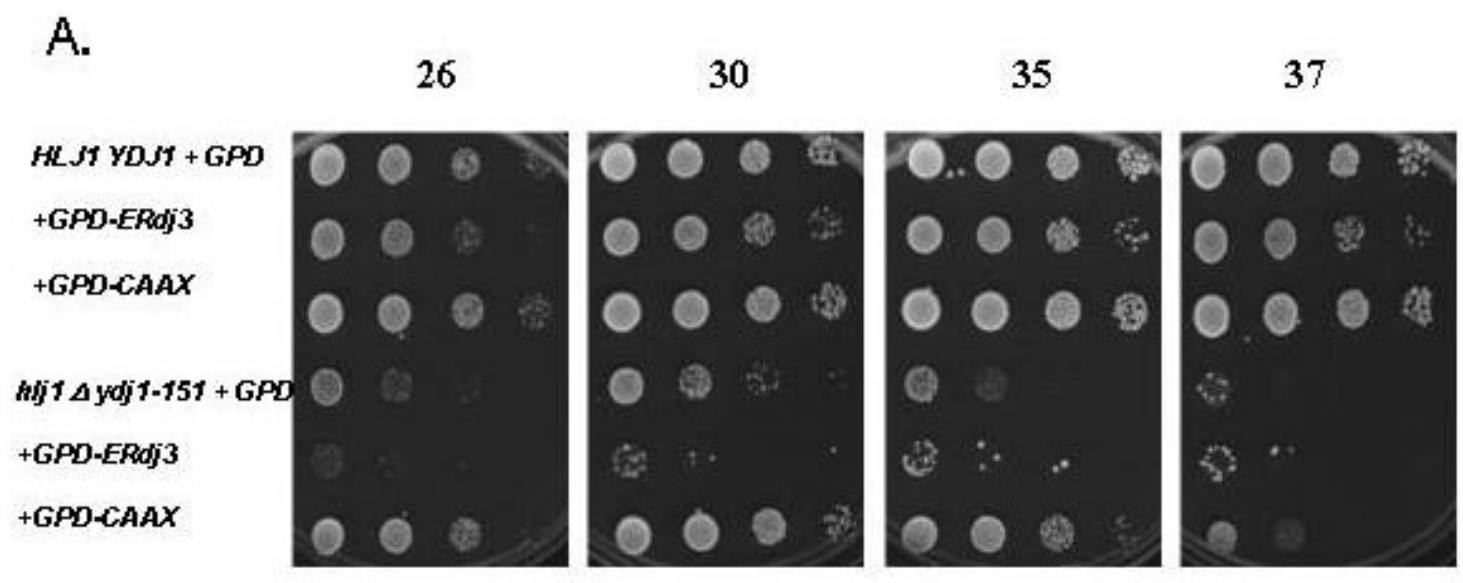

\section{B. ERdj3 stimulates Ssa1's ATPase activity}

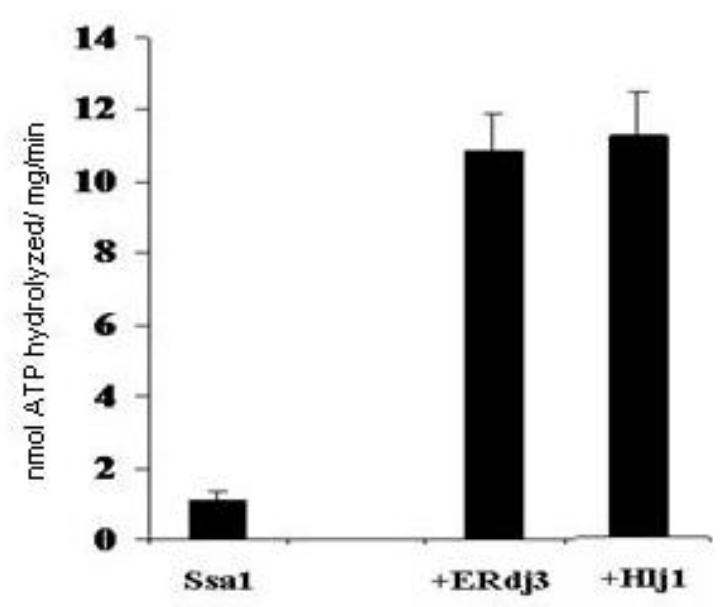

Figure 3-3. Cytosolic expression of ERdj3 rescues the temperature-sensitive growth phenotype of the hlj1 $\Delta y d j 1-151$ strain

(A) an empty vector (GDP), a vector containing an ER-targeted form (ERdj3), or a vector engineered to produce a cytosolically localized form $(\mathrm{CaaX})$ of full-length ERdj3 were expressed in wild-type (HLJIYDJl) and mutant ( $h l j 1 \Delta y d j 1-151)$ yeast strains as described in Figure 3-2A. The plates were incubated for 2 days at the indicated temperatures. (B) ERdj3 robustly stimulates Ssa1's ATPase activity. The ATPase activity of Ssal was measured either by itself or in the presence of ERdj3 or Hlj1 as described in Figure 3-2B. The molar ratio of Ssa1 to the DnaJ proteins is 1:2. 


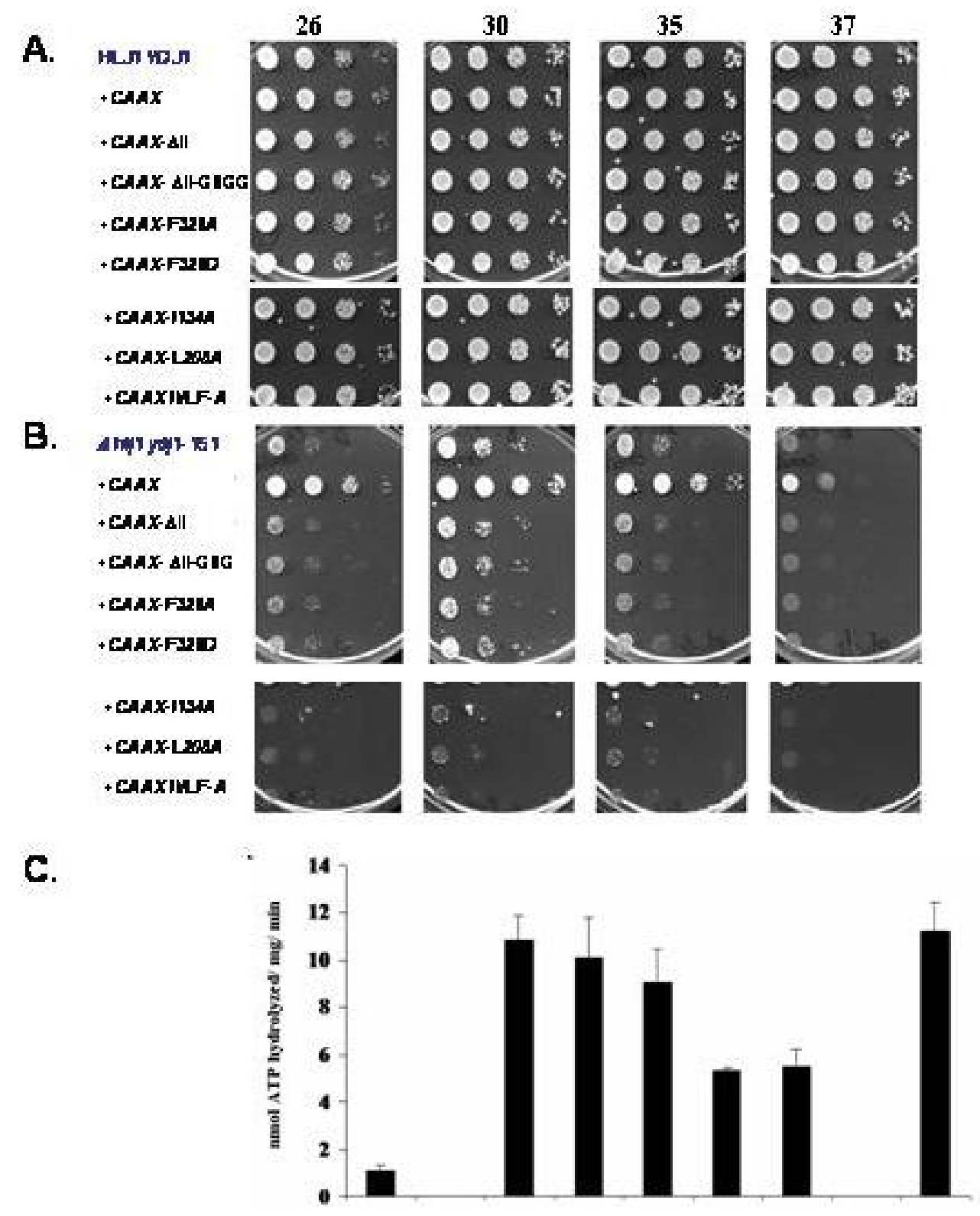

Figure 3-4. ERdj3 substrate binding mutants cannot rescue the temperature sensitive growth phenotype of the $h l j 1 \Delta y d j 1-151$ strain.

Cytosolically forms (CaaX) of wild type or mutant ERdj3 were expressed in wild type (HLJ1YDJ1) (A) or mutant (hlj1 $\Delta y d j 1-151)$ yeast strains (B). They were plated and allowed to grow as described in Firgure 2. (C) All ERdj3 mutants stimulate Ssa1's ATPase activity. The ATPase activity of Ssa1 was measured either by itself or in the presence of the indicated DnaJ proteins as described in Figure 3-2B. The molar ratio of Ssa1 to DnaJ proteins is $1: 2$. 


\subsection{Discussion}

As cochaperones of Hsp70, DnaJ proteins allow Hsp70s to function by stimulating their ATPase activity. The fact that a given Hsp70 in many organelles often has multiple DnaJ cochaperones has led investigators to suggest that association with different DnaJ proteins determines the functional diversity of Hsp70 proteins. We demonstrated that ER-expressed Ydj1 associated with BiP and specific ERdj3 substrates in mammalian cells, which led to a further investigation of the principles regulating the formation of functional DnaJ/Hsp70 pairs. We found that ERdj3 cannot compensate for the loss of yeast Scj1, which is an ER localized Dna J protein. However, ERdj3 could compensate for the loss of Ydj1 when it was expressed in the yeast cytosol. Since ATPase activity is imperative for the function of Hsp70, the failure of ERdj3 to efficiently stimulate the ATPase activity of Kar2p, a Hsp70 in yeast ER, may explain the inability of ERdj3 to compensate Scj1. It was also demonstrated that CaaX-ERdj3 can functionally interact with Ssa1 and compensate the loss of Ydj1. These results suggest that both Ydj1 and ERdj3 can interact with an alternative Hsp70 and must be able to bind to at least some substrates in the other cell type, even though they originate from different organelles in different organisms. In some cases the ability to stimulate Hsp70 ATPase activity is sufficient for many cellular processes (Sahi \& Craig, 2007). Our data suggests this is not sufficient in the case of the ERdj3/Ssa1 pair, as all of the substrate binding mutants were able to stimulate the Ssa1's ATPase activity in vitro but were unable to compensate for the loss of Ydj1 in vivo. This is in contrast to a recent study where growth defects caused by the loss of Ydj1 could be attenuated by the expression of various cytosolic DnaJ proteins or even only their J domains (Sahi \& Craig, 2007). However, unlike present study, their study was done in the background only deleting Ydj1. It is likely that other cytosolic DnaJ proteins (Hlj1) can functionally overlap with Ydj1 in vivo.

In the present study, we found that the $\Delta \mathrm{II}$ mutant retains its ability to stimulate Ssa1 but cannot compensate for the loss of Ydj1, suggesting that deletion of domain II does not grossly affect ERdj3's overall structure and agreeing with previous findings that domain II has an essential role in both Ydj1 and DnaJ function (Szabo et al., 1996; Lu et al., 1998). It was suggested that the cysteines of domain II are important for maintaining the ability of these DnaJ proteins to bind substrate or to cooperate with Hsp70 in substrate folding. In contrast to other type I DnaJ proteins whose cysteine residues form zinc binding sites, the four cysteines that are present in ERdj3 form intra-chain disulfide bonds in domain II. In a recent study using mutational analyses, these cysteines were argued to be essential for the in vitro binding of ERdj3 to denatured thyroglobulin and immunoglobulin (Marcus et al., 2007). Because the yeast cytosol has a more reducing environment than that of the mammalian ER, one might expect that the critical disulfide bonds would not form. The fact that ERdj3 was able to compensate for loss of Ydj1 argues that either the disulfide bonds do form even in the reducing environment or that they are not necessary for function in this context. In support of the first possibility, it has been reported that ER proteins which fold quite stably can form disulfide bonds even in the presence of DTT (Hellman et al., 1999). In addition, we previously demonstrated that reduced ERdj3 can interact not only with BiP but also with substrate in vivo when cells are treated with DTT (Shen et al., 2005). Thus, the requirement for these cysteines in the mutational study might reflect either the presence of altered amino acids in these 
positions or a more stringent requirement for the disulfide bonds in these particular in vitro assays. It is also conceivable that the binding of $\mathrm{Zn}^{2+}$ to the cysteines in domain II of ERdj3 when it is expressed in the yeast cytosol is able to stabilize this domain and that conversely the formation of disulfide bonds in domain II of Ydj1 when it is in the mammalian ER serves a similar purpose.

Previous crystallographic studies of the peptide binding fragment of Ydj1 and Sis1 (Sha et al., 2000; Li et al., 2003; Li \& Sha, 2005) and the mutagenesis study of ERdj3's substrate binding domain (Chapter 1) suggest that type I and type II DnaJ proteins are likely to possess a very similar substrate binding structure. Since the substrate binding domains of Ydj1 and Sis1 are required for robust growth of yeast cells (Johnson et al., 2001), a number of genetic reconstitution studies have been performed (Sahi \& Craig, 2007). Sis 1 , which is a type II family member, is able to complement loss of Ydj1 (Sahi \& Craig, 2007), as was ERdj3 in the present study. However, loss of Sis1 is not complemented by Ydj1 (Luke et al., 1991). Based on our studies showing that substrate binding is important for complementation, it is reasonable to suggest that Sis1 is able to bind Ydj1 substrates that are critical for growth, whereas Ydj1 may not be able to interact with an essential Sis1 substrate. The fact that the actual sequence of domain I of these DnaJ proteins is not highly conserved is consistent with this possibility.

These studies demonstrate that both substrate binding and the ability to stimulate Hsp70 ATPase activity are crucial in the formation of functional DnaJ/Hsp70 pairs. They further suggest that DnaJs exhibit more promiscuity in binding to various substrates than in interaction with Hsp70s. This discovery has implications for models in which different DnaJ proteins allow Hsp70s to contribute to diverse and sometimes opposing cellular functions. 


\section{CHAPTER 4. REGULATED RELEASE OF ERDJ3 FROM UNFOLDED PROTEINS BY BIP ${ }^{1}$}

\subsection{Introduction}

The Hsp70 family of molecular chaperones is a highly conserved, widely expressed, and well-studied group of proteins. These chaperones are found in all organisms where they play a role in every cellular organelle and are essential to nearly all cellular processes. The binding of Hsp70 proteins to non-native structures on a vast array of substrate proteins can serve to stabilize folding intermediates, prevent their aggregation, and aid in protein folding and assembly. This is achieved via direct interaction of the C-terminal substrate binding domain (SBD) of Hsp70 proteins with exposed hydrophobic residues on substrate proteins (Erbse et al., 2004). Peptide binding studies have been performed on BiP (Flynn et al., 1991; Blond-Elguindi et al., 1993), the Hsp70 cognate of the mammalian endoplasmic reticulum (ER), on Hsc 70 (Gragerov \& Gottesman, 1994), a cytosolic mammalian Hsp70, and on DnaK (Rudiger et al., 1997), the $E$. coli family member. Although differences exist, overall the studies suggest that Hsp70 proteins prefer peptides of 5-10 amino acids, which are predominantly hydrophobic in nature, and which have been estimated to occur approximately every 1620 amino acids in the average protein (Flynn et al., 1991). In the case of BiP, the preferred peptides contain hydrophobic amino acids in alternating positions (BlondElguindi et al., 1993). This configuration is most compatible with a preference for extended chains in which the hydrophobic amino acids would be oriented in a single direction to engage the peptide binding pocket of the Hsp70 protein, a possibility that is supported by NMR (Landry et al., 1992) and crystallographic (Zhu et al., 1996) studies.

The binding and release of substrates to the SBD of Hsp70 proteins is tightly regulated by the highly conserved N-terminal nucleotide binding domain (NBD) (Liberek et al., 1991b), which can bind either ATP or ADP. When ATP occupies the cleft of the NBD, the SBD is in an open configuration, which has both a high on and high off rate for unfolded proteins. The hydrolysis of ATP to ADP results in a closure of the lid on the SBD, which stabilizes the interaction with bound proteins. Discharge of the unfolded protein occurs when ADP is released and exchanged for ATP. This reopens the lid on the SBD, which allows the bound substrate to be released and provides an opportunity for it to fold. A number of recent studies shed light on the interaction between the two domains, which controls the activity of this group of chaperones (Jiang et al., 2005; Vogel et al., 2006a; Vogel et al., 2006b; Liu \& Hendrickson, 2007; Awad et al., 2008).

The Hsp70 ATPase cycle, which is essential to the chaperoning process, is controlled by a number of co-factors that regulate either ATP hydrolysis or nucleotide exchange. DnaJ was originally identified along with DnaK (Hsp70) in a genetic screen in E. coli for genes that are required for DNA replication (Saito \& Uchida, 1977; Yochem et al., 1978). Later it was shown that DnaK and DnaJ are in the same genetic pathway and

\footnotetext{
${ }^{1}$ Chapter 4 modified from an article accepted for publication by EMBO Journal (Jin Y, Awad W, Petrova $\mathrm{K}$, and Hendershot LM, Regulated release of ERdj3 from unfolded proteins by BiP. EMBO J. In press.).
} 
that DnaJ stimulates the ATPase activity of DnaK, thereby stabilizing the binding of DnaK to substrates (Liberek et al., 1991a). As is the case with Hsp70s, DnaJ proteins are present in all organisms and all organelles, and the number of DnaJ proteins in an organism often exceeds the number of Hsp70 proteins present (Caplan et al., 1993; Cheetham \& Caplan, 1998). DnaJ proteins all possess a highly conserved $\sim 70$ amino acid "J" domain, which contains an invariant tripeptide sequence, His-Pro-Asp, that is required to interact with the ATP-bound form Hsp70 proteins (Mayer et al., 1999). Like Hsp70 proteins, at least some DnaJ proteins can bind directly to unfolded substrates (Cheetham et al., 1998; Fan et al., 2003). Peptide binding studies for E. coli DnaJ revealed significant overlap with the peptides that bound DnaK (Rudiger et al., 2001), arguing that DnaJ was likely to also bind to extended hydrophobic residues on unfolded proteins. This possibility was supported by crystallographic data obtained for a peptide bound to the yeast cytosolic DnaJ protein, Ydj1 (Li et al., 2003). The fact that DnaJs specifically interact with the ATP bound form of Hsp70s led to a model (Walter \& Blobel, 1983; Mayer et al., 1999) where DnaJ proteins would bind first to unfolded proteins, recruit the ATP-bound or "open" form of Hsp70 to the substrate, and then stimulate its ATPase activity to "close" it onto the substrate more stably. This model was supported by data showing that a cytosolic DnaJ protein bound to nascent chains extruding from the ribosome before the Hsp70 protein did (Hendrick et al., 1993), and by in vitro binding studies with DnaK, DnaJ, and denatured luciferase (Szabo et al., 1994). However, these studies did not reveal how DnaJ proteins were released from the substrate. Unlike Hsp70, DnaJ proteins do not bind to nucleotide and have not been demonstrated to exist in different conformational states.

The mammalian ER possesses at least six DnaJ family members (Brightman et al., 1995; Meyer et al., 2000; Tyedmers et al., 2000; Yu et al., 2000; Shen et al., 2002; Cunnea et al., 2003; Rutkowski et al., 2007; Petrova et al., 2008). One of these, ERdj3, was shown previously by our group to bind to a number of unfolded proteins in the ER that were BiP substrates (Shen and Hendershot, 2005). When the binding of wild-type and mutant (HPD $\rightarrow$ QPD) ERdj3 to several different substrates was compared, we consistently found that mutant ERdj3 bound quantitatively better and longer than wildtype ERdj3 (Shen et al., 2005). The present study was undertaken to better understand the requirements for releasing DnaJ proteins from substrates. Using a series of ERdj3 and BiP mutants, we found that release of ERdj3 was not simply due to a competition or exchange with $\mathrm{BiP}$, but that a functional interaction between ERdj3 and BiP was required. We hypothesize that in addition to the ERdj3-induced conformational change that occurs in BiP, which stabilizes BiP's binding to the unfolded protein, that there is a reciprocal change in the conformation of ERdj 3 that triggers its release from substrate.

\subsection{Materials and Methods}

\subsubsection{Cell culture, transfection, and immunoprecipitation}

COS-1 monkey kidney fibroblast cells were maintained in DMEM supplemented with $10 \%$ fetal bovine serum, $2 \mathrm{mM}$ L-glutamine, and $1 \%$ Fungisone in $3 \% \mathrm{CO}_{2}$. Cells 
were transfected with the indicated vectors using the Fugene 6 transfection reagent (Roche Diagnostics), and after forty-eight hours post-transfection, cells were labeled with

${ }^{35} \mathrm{~S}$ Translabel (Amersham Biosciences) for 3h. Cells were treated with $150 \mu \mathrm{g} / \mathrm{ml} \mathrm{3,3'-}$ dithio-bis (propionic acid N-hydroxysuccinimide ester) (DSP), a membrane permeable cross-linking reagent (Sigma-Aldrich) for $1 \mathrm{~h}$ on ice. Cell lysates were prepared using an NP40 lysing buffer and immunoprecipitated with indicated antisera followed by binding to Protein A Sepharose beads. The immunoprecipitated complexes were analyzed by SDS-PAGE under reducing conditions, and the signal was detected using Amplify (Amersham Bioscience) for radiographic visualization.

\subsubsection{In vitro translation and heavy chain binding assay}

Ag8.8 murine plasmacytoma cells were maintained in RPMI 1640 supplemented with $10 \%$ fetal bovine serum, $2 \mathrm{mM}$ L-glutamine, and 1\% Fungizone in 5\% CO2. Cells were metabolically labeled for $16 \mathrm{~h}$ with ${ }^{35} \mathrm{~S}$ Translabel (Amersham Biosciences) and treated with or without DSP prior to lysing. Cell lysates were prepared and Ig heavy chains were isolated by binding to Protein A as described previously (Wei et al., 1995). To release BiP from heavy chains, non-cross-linked samples were supplemented with 1 $\mathrm{mM} \mathrm{MgCl} 2,25 \mathrm{mM} \mathrm{KCl}$, and $1 \mathrm{mM} \mathrm{ATP}$. These samples are the source of free HC used in the in vitro binding assays, except that the beads were washed an additional three times in PBS to reduce detergent in the samples, which interferes with the ability of ERdj 3 to remain associated with unfolded substrates (Shen et al., 2005).

The cDNAs encoding wild type and mutant (H35Q) ERdj3 were transcribed from the T7 promoter of 3HADSL-ERdj3 (Stratagene) and translated using ${ }^{35} \mathrm{~S}$ methionine (Amersham Biosciences) and the TNT coupled rabbit reticulocyte lysate (Promega). Equivalent counts for the two protein products were loaded directly on reducing SDSpolyacrylamide gels or incubated with either uncoupled Protein A Sepharose beads (washed three times in PBS), or with the Protein A Sepharose beads to which free $\gamma \mathrm{HC}$ were bound. After incubating for $1 \mathrm{~h}$ on ice, the beads were washed three times with PBS and bound proteins were subjected to reducing SDS-PAGE.

\subsubsection{IP western}

Firefly luciferase (Promega) was left untreated (N) or heat denatured (D) at $42^{\circ} \mathrm{C}$ for $1 \mathrm{~h}$. In both cases, $0.5 \mu \mathrm{g}$ of protein was incubated with recombinant wild type ERdj3 protein $(2.0 \mu \mathrm{g})$ in PBS and immunoprecipitated with either anti-ERdj3 polyclonal antiserum followed by protein A Sepharose beads or with protein A Sepharose beads alone. Bound proteins were subjected to reducing SDS-PAGE and then transferred to a PVDF membrane (BIO-RAD), which was blotted with an anti-luciferase antiserum (1:1000) (Promega). Donkey anti-goat Ig conjugated to HRP (1:5000) was used as a secondary antibody and the signal was detected by chemiluminescence (ECL). 


\subsubsection{Protein purification}

Expression of His-tagged wild-type and mutants ERdj3 was induced in E. coli M15 cells with $0.1 \mathrm{mM}$ isopropyl $\beta$-D-thiogalactoside (Sigma) followed by growth for $18 \mathrm{~h}$ at $18^{\circ} \mathrm{C}$. The recombinant proteins were purified on $\mathrm{Ni}^{2+}$-agarose columns under nondenaturing conditions (Qiagen QIAexpress system), dialyzed in $25 \mathrm{mM}$ sodium phosphate buffer ( $\mathrm{pH} 7.0$ ), containing $150 \mathrm{mM} \mathrm{NaCl}, 0.02 \%$ Triton X-100, 50\% glycerol, and a protease inhibitor cocktail (Roche), and stored at $-20^{\circ} \mathrm{C}$. His-tagged wild-type and mutant BiP proteins were induced with $1 \mathrm{mM}$ IPTG for $2 \mathrm{~h}$ at $37^{\circ} \mathrm{C}$, purified on $\mathrm{Ni}^{2+}-$ agarose columns, dialyzed and stored in 20mM HEPES buffer ( $\mathrm{pH} 7.2$ ) with $50 \mathrm{mM} \mathrm{KCl}$, $5 \mathrm{mM} \mathrm{MgCl}_{2}, 0.01 \% \mathrm{NP}-40,50 \%$ glycerol, and a protease inhibitor cocktail.

\subsubsection{Measurement of complex formation between ERdj3 proteins and denatured luciferase}

Firefly luciferase was denatured in buffer containing 7M Urea, 25mM HEPES (pH 7.5), $50 \mathrm{mM} \mathrm{KCl}, 5 \mathrm{mM}$ EDTA, $5 \mathrm{mM} \mathrm{MgCl}_{2}$ and $5 \mathrm{mM}$ dithiothreitol at room temperature for 40 min and then diluted into PBS containing 0.05\% BSA (final concentration 0.5 $\mu \mathrm{g} / \mathrm{ml}$ ). $100 \mu \mathrm{l}$ of this solution was added into each well of a 96-well microtiter plate purchased from Theromo (Immulon 2HB Flat Bottom Microtiter Plate) and allowed to bind overnight at $4^{\circ} \mathrm{C}$. Wells were washed with PBS and blocked with $200 \mu \mathrm{PBS}$ containing $1 \% \mathrm{BSA}$ for $1 \mathrm{~h}$ at room temperature. The indicated concentrations of wild type or mutant ERdj3 in $100 \mu \mathrm{l}$ of PBS with $0.05 \%$ BSA was added to the wells and incubated for $1 \mathrm{~h}$ at room temperature, followed by washing with PBS to remove unbounded ERdj3. The amount of ERdj3 that remained bound to denatured luciferase was detected with a polyclonal anti-ERdj3 antiserum (Shen et al., 2005) followed by donkey anti-rabbit Ig conjugated to alkaline phosphatase (Promega). 4-Nitrophenyl phosphate disodium salt hexahydrate (Sigma) was added, and after stopping the reaction with $0.75 \mathrm{M} \mathrm{NaCl}$, the plates were read on a spectrophotometer at wavelength $405 \mathrm{nM}$. Negative controls were set up for each plate in wells that did not contain either denatured luciferase, ERdj3, or each of the antibodies, but which included all the other steps of the reaction.

\subsubsection{Release of ERdj3 from luciferase}

To detect the amount of BiP binding to denatured luciferase, recombinant hamster BiP was added to wells coated with denatured luciferase instead of ERdj3, and incubated

as above, except that a rabbit polyclonal anti-rodent BiP antiserum was used to detect BiP binding. To test the ability of wild-type and mutant BiP to release ERdj3 from luciferase, ERdj3 was first bound to luciferase as above. After washing away unbound ERdj3, the indicated amounts of recombinant BiP proteins were added to the wells in PBS containing $1 \mathrm{mM} \mathrm{MgCl}_{2}, 25 \mathrm{mM} \mathrm{KCl}$ and either $1 \mathrm{mM}$ ATP or no nucleotide. The plates were incubated for $1 \mathrm{hr}$ at room temperature, and the amount of ERdj3 or BiP associated with the denatured luciferase was determined as above. 


\subsubsection{ATPase assay}

ATPase assays were performed as described previously (Chevalier et al., 1998). Briefly, $1 \mu \mathrm{M}$ of the various recombinant BiP proteins was incubated alone or with 0.5 $\mu \mathrm{M}$ of the indicated full length ERdj3 proteins at $37^{\circ} \mathrm{C}$ for $20 \mathrm{~min}$ in ATPase buffer containing $\left[\gamma_{-}^{32} \mathrm{P}\right]$ ATP (PerkinElmer). After chromatography, the radioactive ATP and free phosphate signals were quantified by phosphoimager analysis (Molecular Dynamics, Sunnyvale, CA) using Image Quant software. The free phosphate signal was expressed as a percent of the total phosphate signal. Data were deduced from three independent experiments, and the error bars represent standard deviations (S.D.).

\subsection{Results}

\subsubsection{Comparison of the effects of $J$ domain mutations on ERdj3's ability to associate with substrate both in vivo and in vitro}

It has been shown that the $\mathrm{J}$ domains of DnaJ proteins are important for interactions with their Hsp70 partners, where the signature HPD motif in the J domain plays an indispensable role. We previously demonstrated that a QPD mutation in ERdj3 abrogated its ability to interact with its ER Hsp70 partner-BiP both physically and functionally (Shen et al., 2005). To determine whether the interaction with BiP was crucial for ERdj3's ability to bind to unfolded substrates, we examined the ability of wild-type and mutant ERdj3 to bind to immunoglobin heavy chain $(\gamma \mathrm{HC})$ both in vivo and in vitro. First, we co-expressed $\gamma \mathrm{HC}$ along with HA-tagged versions of either wildtype ERdj3 or two different J domain mutants (both QPD and $\Delta \mathrm{J}$ ) in COS cell. Coimmunoprecipitation experiments were performed on DSP-cross-linked cell lysates. We found that $\mathrm{J}$ domain mutations did not negatively affect the ability of the mutant ERdj3 proteins to bind to $\gamma \mathrm{HC}$ in vivo (Figure 4-1A). In fact, in both cases there was actually more binding of the QPD and $\triangle \mathrm{J}$ mutants to the $\gamma \mathrm{HC}$ as compared to the binding of wildtype ERdj3. Wild type and mutant ERdj3 proteins were also expressed in COS cells alone. Protein A Sepharose beads did not precipitate any of three proteins (Figure B-1) demonstrating that the binding observed in Figure 4-1A is dependent on the coexpression of $\gamma \mathrm{HC}$. These data suggested two things; first, since these mutants are unable to interact with BiP, ERdj3 might bind directly to unfolded substrates, and second these ERdj3 mutants might have a higher affinity for substrate or some component of the ER chaperone complex (Meunier et al., 2002). To directly test the first possibility, we developed an in vitro assay to examine the binding of ERdj3 to $\gamma \mathrm{HC}$ in the absence of other resident ER chaperones and folding enzymes. It was based on our previous demonstration that $\mathrm{BiP}$ can be released from isolated $\mathrm{HC}$ in vitro with ATP leaving the $\mathrm{HC}$ in a conformation that allows them to reassociate with exogenously added BiP (Wei et al., 1995). As shown in Figure 4-1B (lane 1), ERdj3 can be isolated with $\gamma \mathrm{HC}$ only when cells are pretreated with a membrane permeable crosslinker, DSP, whereas BiP's association with $\gamma \mathrm{HC}$ is detectable even without crosslinking (Figure 4-1B, lane3). However, addition of ATP releases BiP from the $\gamma \mathrm{HC}$ (Figure 4-1B, lane2). These free $\mathrm{HC}$ were used for binding to in vitro translated ERdj3 proteins (Figure 4-1C). We found 
A.

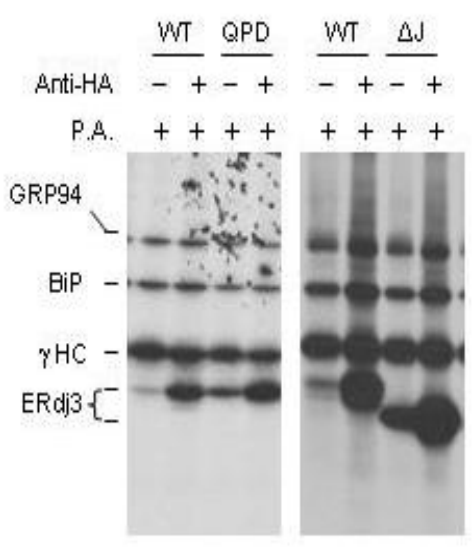

B.

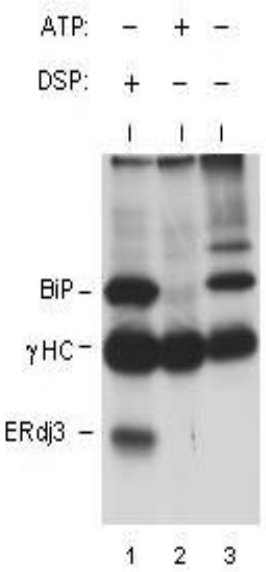

C.

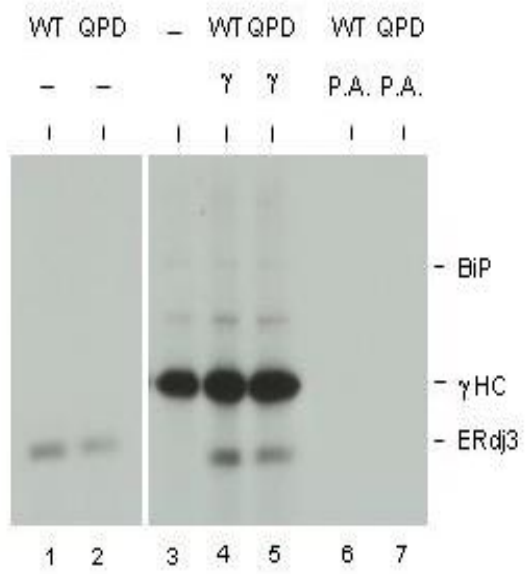

Figure 4-1. ERdj3 binds to $\gamma \mathrm{HC}$ directly

(A) Cos cells were co-transfected with cDNAs encoding $\gamma \mathrm{HC}$ and the indicated HAtagged ERdj3 constructs. Metabolically labeled, cross-linked cell lysates were immunoprecipitated with anti-HA or Protein A Sepharose alone. Isolated proteins were separated by reducing SDS-PAGE. (B) Ag8.8 cells were metabolically labeled for $16 \mathrm{~h}$ with ${ }^{35} \mathrm{~S}$ methionine and cysteine and incubated with (lane 1) or without (lanes 2 and 3 ) DSP. Cell lysates were prepared with (lane 2) or without (lanes 1 and 3) ATP and immunoprecipitated with Protein A Sepharose. (C) Wild-type (WT) and QPD mutant (Mut) ERdj3 were in vitro translated and run directly (lanes 6 and 7) or incubated with the free $\gamma \mathrm{HC}$ immobilized on protein A Sepharose beads prepared as in lane 2 in Figure 4-1B (lanes 2 and 3) or with protein A beads alone (lanes 4 and 5). 
that unlike the in vivo binding assays, the QPD mutant bound to $\gamma \mathrm{HC}$ at similar levels as observed for wild-type ERdj3 (Figure 4-1C, lanes 4 and 5). These data revealed that ERdj3 associates directly with substrates and also argues that the enhanced binding of ERdj3 mutants to $\gamma \mathrm{HC}$ in vivo is unlikely to be due to their having a higher affinity for substrate. Instead, it suggested that something else in the cell might be contributing to the difference between wild-type and mutant ERdj3's association with substrate. Based on our previous data showing that mutant ERdj3 remains bound to unfolded Ig light chains much longer than wild-type ERdj3 (Shen et al., 2005), we hypothesized that the J domain mutations might be affecting release of the ER DnaJ proteins from substrates and that release might be dependent on a functional interaction with BiP.

\subsubsection{Development of an in vitro system to detect binding and release of ERdj3 from substrates}

Because the isolation of free $\mathrm{HC}$ was somewhat cumbersome, we wished to develop a simpler in vitro binding assay. We chose denatured firefly luciferase (D-Luc), because it has been widely used as an in vitro substrate for other DnaJ family members, including E. coli DnaJ and two yeast cytosolic DnaJ family members Sis1 and Ydj1 (Szabo et al., 1994; Schumacher et al., 1996; Lu \& Cyr, 1998a; Lu \& Cyr, 1998b). To determine if denatured luciferase could serve as an ERdj3 substrate in vitro, we examined the ability of wild-type ERdj3 to bind to either native or heat denatured luciferase in solution. For these experiments we denatured luciferase with heat instead of urea, because we did not want to interfere with the protein: protein interactions required for association and for immunoprecipitation. ERdj3 was allowed to interact with native or denatured luciferase and the samples were immunoprecipitated with either a polyclonal anti-ERdj3 antiserum or Protein A Sepharose beads. The association of luciferase was determined by immunoblotting with an anti-luciferase antibody. We found that indeed the binding of ERdj 3 to denatured luciferase (D) was readily detectable, whereas its binding to native luciferase $(\mathrm{N})$ was below the level of detection (Figure 4-2A). This distinction in binding is in keeping with ERdj3 acting as a chaperone and demonstrated that denatured luciferase could be used as an in vitro substrate for ERdj3.

Next a modified ELISA was developed, which would allow us to readily examine the ability of ERdj3 to bind to luciferase under multiple conditions. For this assay, the luciferase was chemically denatured, as has been done in a number of other studies (Szabo et al., 1994; Lu et al., 1998a). First, we tested the binding of wild-type ERdj3 and the QPD mutant to chemically denatured luciferase which was bound to 96-well plates. Similar to the in vitro binding of these proteins to $\gamma \mathrm{HC}$, we found that both wild-type ERdj3 and the QPD mutant associated with chemically denatured luciferase equally (Figure 4-2B). Thus, mutation of HPD sequence to QPD did not affect the binding of ERdj3 to chemically denatured luciferase. 
A.

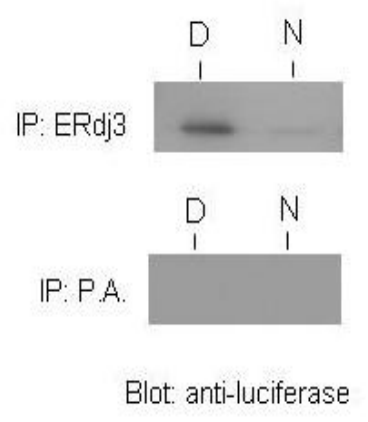

B.

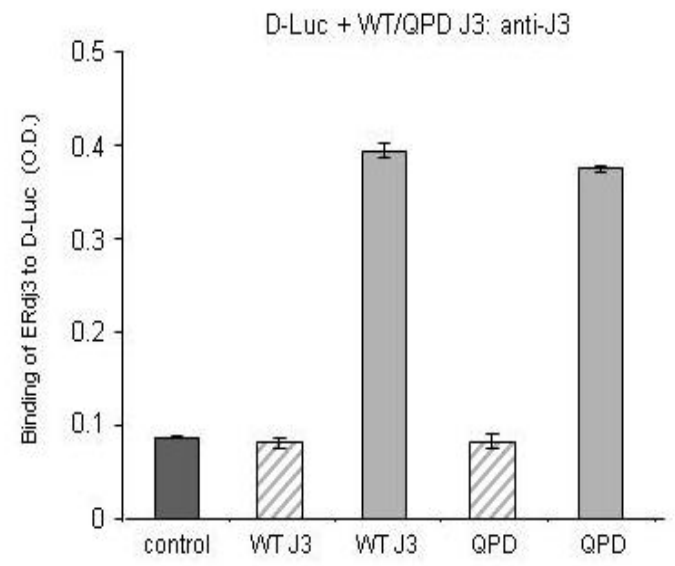

Figure 4-2. WT and QPD ERdj3 bind to denatured luciferase similarly in vitro

(A)Temperature denatured (D) or native (N) luciferase (Luc) was incubated with recombinant wild type ERdj3 protein and then immunoprecipitated with either antiERdj3 polyclonal antiserum or with protein A Sepharose beads alone. Reaction cocktails were subjected to reducing SDS-PAGE and then transfer to a PVDF membrane. The membrane was blotted with goat anti-luciferase antiserum followed by donkey anti-goat Ig conjugated to HRP. The signal was detected by chemilluminesence. (B) Chemically denatured luciferase (grey bars) or binding buffer alone (hatched bars) was used to coat 96-well plates. After washing and blocking with $1 \%$ BSA, recombinant wild-type or the QPD mutant ERdj3 proteins $(0.5 \mu \mathrm{M})$ were added to the wells and incubated for $1 \mathrm{hr}$ at RT. After washing, bound ERdj3 was detected with a polyclonal anti-ERdj3 antiserum, followed by donkey anti-rabbit Ig conjugated to alkaline phosphatase. The DNTP substrate was added and after developing, the plates were read on a spectrophotometer and the signal was expressed in OD units. A luciferase coated well that did not receive ERdj3 protein was treated similarly and serves as a negative control for the antibody (dark grey). All samples were run in triplicate and error bars are indicated. 


\subsubsection{ATP does not affect the binding of wild type ERdj3 or the QPD and HPN mutants to substrate in vitro}

Before testing our hypothesis that ERdj3 release from substrates occurs in response to a functional interaction with $\mathrm{BiP}$, it was necessary to set up an assay to saturate the binding of ERdj3 to luciferase and to ensure that incubation with ATP did not affect this binding. Increasing concentrations of wild-type (Figure 4-3A), QPD (Figure 4-3B), or HPN (Figure 4-3C) recombinant ERdj3 proteins were added to luciferase coated wells in the absence (Figure 4-3 solid bar) or presence (Figure 4-3 stippled bar) of ATP. We found that all three proteins reached saturation binding at concentrations of $\sim 500 \mathrm{nM}$ and that the inclusion of ATP in the binding buffer did not affect ERdj3's ability to bind to substrate. Thus, in the following experiments $500 \mathrm{nM}$ ERdj3 was used.

\subsubsection{BiP promoted the release of wild-type ERdj3 from chemically denatured luciferase in the presence of ATP}

If a functional interaction between BiP and ERdj3 is critical for releasing ERdj3 from substrates, the amount of ERdj3 that is associated with substrate is expected to decrease in the presence of BiP in an ATP-dependent manner. To examine this possibility, increasing amounts of BiP were added to wells containing ERdj3 bound to denatured luciferase in the absence and presence of ATP (Figure 4-4A). We found that the addition of increasing amounts of BiP in the absence of ATP did not affect ERdj3's association with denatured luciferase (Figure 4-4A, solid bar), although there was a detectable increase in the binding of $\mathrm{BiP}$ to the substrate (Figure 4-4B, solid bar). This demonstrates that release of ERdj3 does not occur via a simple competition between these two chaperones for substrate and further suggests that BiP and ERdj3 binding sites are not completely overlapping. However, when ATP was included with BiP, we found that the ability of BiP to release ERdj3 was dependent not only on the concentration of $\mathrm{BiP}$, but also required ATP (Figure 4-4A stippled bar). The binding of BiP to denatured luciferase did not increase (Figure 4-4B stippled) when ERdj3 was released (Figure 4-4A stippled bar), again suggesting that ERdj3 release does not occur due to a simple competition with BiP. To examine this from the other direction, we first bound either wild-type BiP or a BiP mutant that cannot interact with ER DnaJ proteins $(\mathrm{R} 197 \mathrm{H})$ (Awad et al., 2008) to denatured luciferase and measured the ability of ERdj3 to release them. We found that the addition of ERdj3 did not induce a reduction in the binding of either wild-type or mutant BiP to luciferase coated well (Figure 4-4C solid and stippled bars), even though we could readily measure the binding of ERdj3 to luciferase (Figure 4-4C checkered bars). This further argues that the release of ERdj3 does not occur due to a simple competition between $\mathrm{BiP}$ and ERdj3 for binding sites on the substrate.

\subsubsection{BiP mutants that do not interact with ERdj3 failed to promote the release of ERdj3 from substrate}

The requirement of ATP for BiP to release ERdj3 from the substrate suggested that a functional interaction between BiP and ERdj3 might be necessary. To test this 
A.

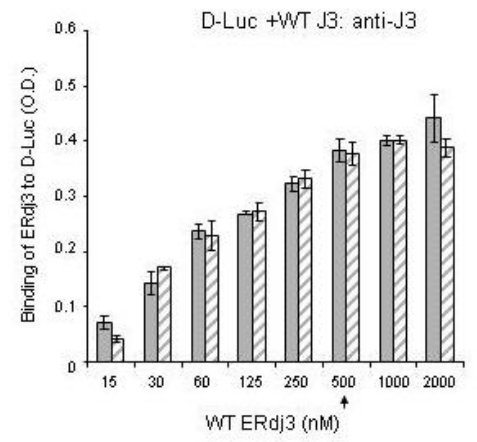

B.

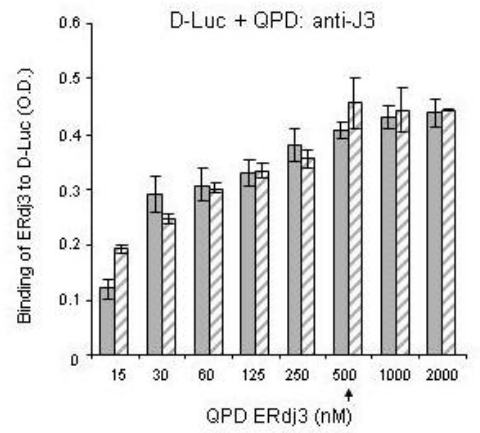

C.

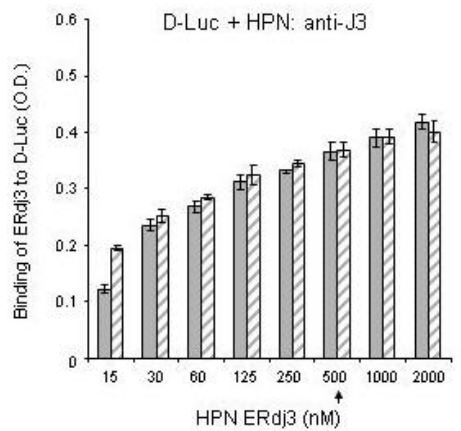

Figure 4-3. WT and mutant (QPD and HPN) ERdj3 bind to D-Luc similarly and ATP does not affect their binding

Chemically denatured luciferase was used to coat the wells and the indicated amounts of WT (A) QPD (B) or HPN (C) ERdj3 were added to the wells with (stippled bar) or without (solid bar) ATP. The plates were developed with anti-ERdj3 as described in Figure 4-2. The arrow indicates the concentration of ERdj3 that was used in the following experiments. 

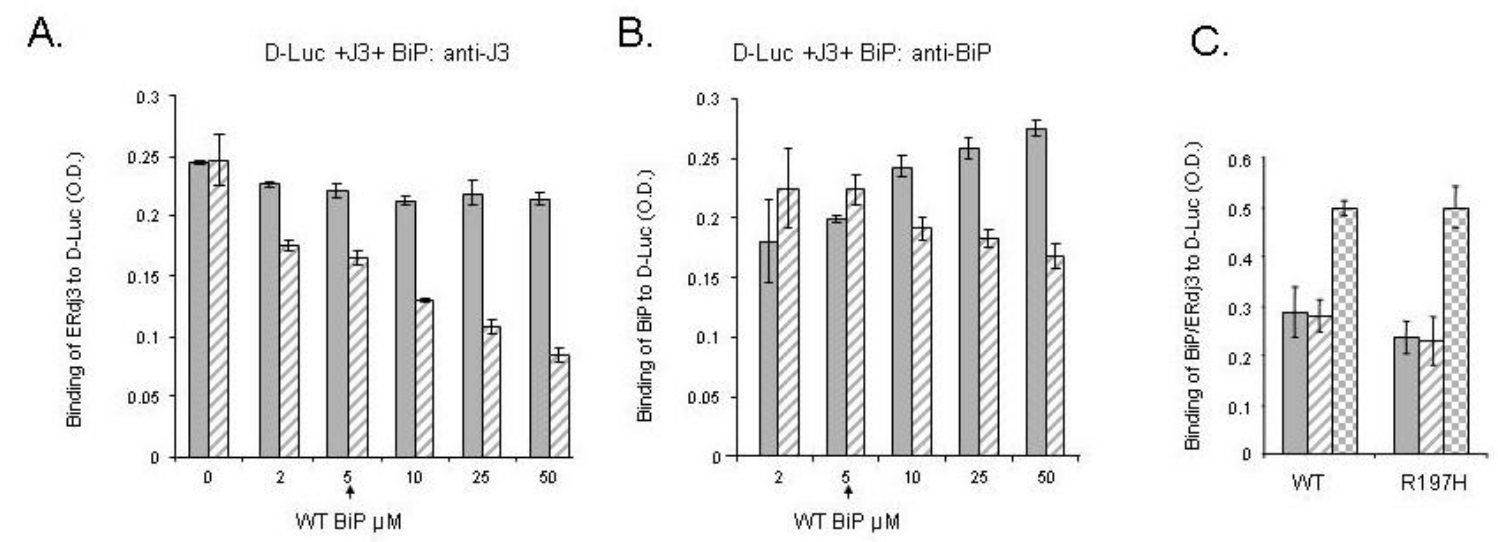

\section{Figure 4-4. BiP releases ERdj3 from D-Luc in an ATP-dependent manner}

(A) Chemically denatured luciferase was added to the wells followed by ERdj3 binding as described previously. After washing, the indicated amounts WT BiP were added to the ERdj3:luciferase complexes with (stippled bar) or without (solid bar) ATP and incubated for an additional $1 \mathrm{hr}$ at room temperature. After washing, the amount of ERdj 3 that remained bound to luciferase was detected with an anti-ERdj3 antiserum. (B) On a parallel plate the amount of $\mathrm{BiP}$ that was associated with luciferase was determined by incubating with an anti-BiP antiserum. (C) Either wild-type or mutant (R197H) BiP was allowed to bind to luciferase. After washing, ERdj3 was added to half the wells and the amount of BiP that was bound without ERdj3 (solid bars) or with ERdj3 (stippled bars) was measured with an anti-BiP antiserum. In a parallel set of wells, ERdj3 binding was measured with an anti-ERdj3 antibody (checkered bars). 
possibility, we examined the ability of a number of different $\mathrm{BiP}$ mutants to release ERdj3 from luciferase. A highly conserved arginine on the ATPase domain of Hsp70 proteins (R197 in BiP) has been shown to be essential for interaction with the HPD motif on DnaJ proteins (Gassler et al., 1998; Suh et al., 1998; Alder et al., 2005). We recently made three substitutions at this site (R197H, R197A, and R197E), all of which have ATPase activity equal to or greater than wild-type BiP, but none of them can bind or be further stimulated by J proteins (Awad et al., 2008). Two other BiP mutants were also tested; a G227D mutant that cannot bind to ATP and a T37G mutant that cannot undergo the ATP-induced conformational change that is required for its chaperoning activity (Wei et al., 1995). Recombinant proteins corresponding to each of these mutants were made and tested both for their ability to bind to luciferase and to release wild-type ERdj3. We found that all five $\mathrm{BiP}$ mutants were able to bind equivalently to luciferase when tested at a concentration of $50 \mu \mathrm{M}$ (Figure 4-5A), whereas only very low levels of background binding to the wells were observed for all of these proteins when luciferase was not present (Figure B-2). Although all of the mutants bound to luciferase both alone and in the presence of ERdj3 (Figure 4-5C), none of them was able to release ERdj3 from this substrate even in the presence of ATP (Figure 4-5B).

\subsubsection{Wild type BiP does not release two ERdj3 mutants, QPD and HPN, from luciferase}

To further explore the possibility that a functional interaction between ERdj3 and $\mathrm{BiP}$ was required for ERdj3 release, we produced two ERdj3 proteins in which the HPD motif had been mutated and therefore should not interact functionally with wild-type BiP based on previously defined DnaJ mutants (Wall et al., 1994). The first of these, $\mathrm{HPD} \rightarrow \mathrm{QPD}$, disrupts binding to BiP and stimulation of its ATPase activity without interfering with the ability of this mutant to bind to substrates (Shen et al., 2005). The second mutant HPD $\rightarrow$ HPN corresponds to a DnaJ mutant that was defective in interacting with wild-type DnaK (Suh et al., 1998). We first tested the ability of these two mutants to bind to BiP in vivo and to stimulate its ATPase activity in vitro. Wildtype and mutant ERdj3 proteins were co-expressed in COS cells with BiP. Forty-eight hr post-transfection metabolically labeled cells were incubated with DSP to cross-link proteins and cell lysates were immunoprecipitated with either anti-BiP or anti-ERdj3 polyclonal antiserum (Figure 4-6A). We found that both mutations interfered with the ability of ERdj3 to bind to BiP, which is in keeping with data from a number of other DnaJ family members (Tsai \& Douglas, 1996; Kelley \& Georgopoulos, 1997; WittungStafshede et al., 2003). Recombinant full-length proteins corresponding to these mutants were produced, and their ability to stimulate the ATPase activity of BiP was compared to that of wild-type ERdj3. We found that wild-type ERdj3 stimulated BiP's ATPase activity about 2-fold, which is in keeping with previous data obtained with only the $\mathrm{J}$ domain and glycine/phenylalanine regions of ERdj3 (Shen et al., 2005), whereas the HPN and QPD mutants were unable to appreciably increase the hydrolysis of ATP (Figure 4-6B).

We next performed an experiment similar to those described in Figures 4-4 and 4-5, except that this time we were asking if wild-type BiP was only capable of releasing 
A.

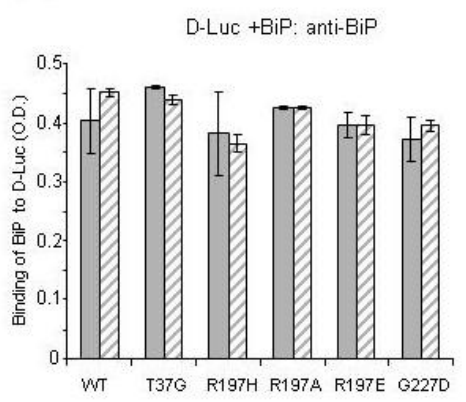

B.

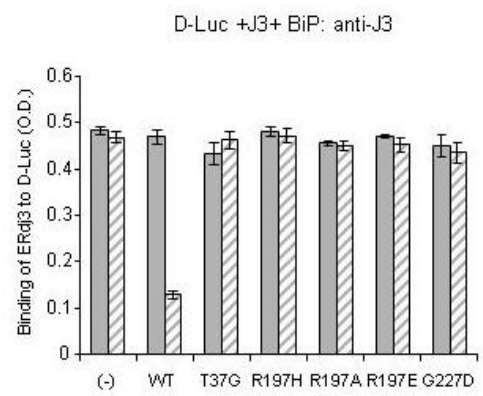

C.

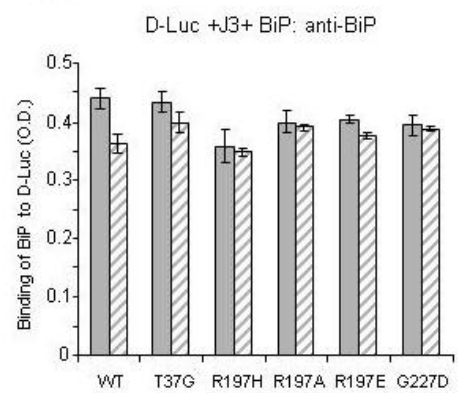

Figure 4-5. Only WT BiP releases ERdj3 from D-Luc

(A) Chemically denatured luciferase was added to the wells, which were then incubated with wild-type or mutant BiP. The binding of BiP to D-Luc was performed in the absence of ERdj3 and detected with anti-BiP serum. (B and C) Denatured luciferase was added to wells and wild-type ERdj3 was allowed to bind as described. After washing, either wild-type or mutant BiP was added with (stippled bars) or without (solid bars) ATP. The amount of ERdj3 remaining was detected with an anti-ERdj3 antiserum (B) and the binding of $\mathrm{BiP}$ was detected with an anti-BiP antiserum $(\mathrm{C})$ and expressed in O.D. units. 
A.

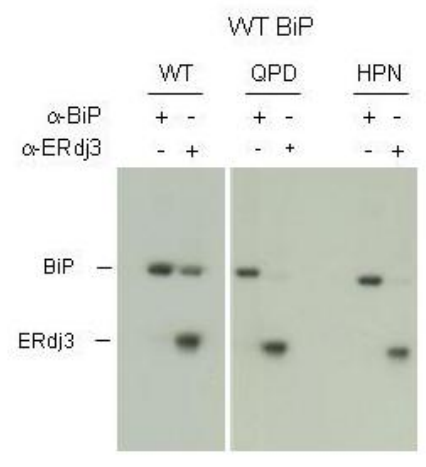

B.

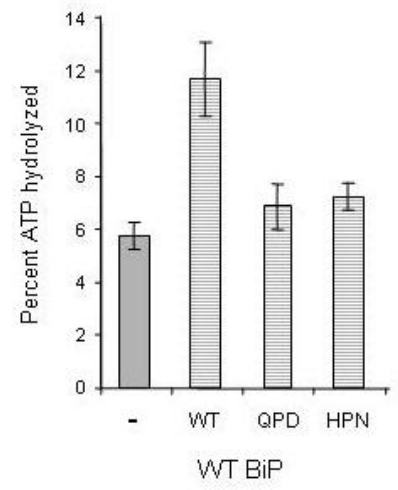

C.

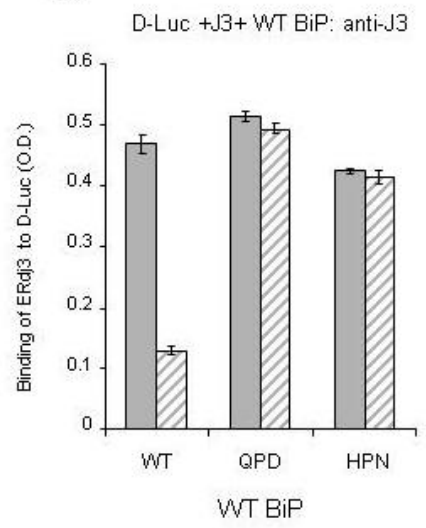

Figure 4-6. Wild-type BiP can only release wild-type ERdj3 from D-Luc

(A) Cos cells were co-transfected with wild-type BiP and the indicated ERdj3 vectors. DSP-cross-linked cell lysates were immunoprecipitated with anti-BiP or anti-ERdj3 and analyzed by reducing SDS-PAGE. (B) ATPase assays were performed on wild-type BiP alone or with a 4-fold molar excess of wild-type, HPN, or QPD ERdj3. ATP hydrolysis was measured by quantitating ADP and expressing it as a percent of total nucleotide. (C) An experiment similar to that described in the previous figure was performed, except that either wild-type or mutant ERdj3 was bound to luciferase first. After washing, wild-type BiP was added with (stippled bars) or without (solid bars) ATP. The amount of ERdj3 that remained bound was detected with an anti-ERdj3 antiserum and expressed in O.D. units. 
wild-type ERdj3 or if it was also able to release the two ERdj3 mutants that did not functionally interact with $\mathrm{BiP}$. If BiP released the ERdj3 mutants, it would argue that a functional $\mathrm{BiP} / \mathrm{ATP} /$ substrate interaction was required but that there was no need for a functional BiP/ERdj3 interaction. We found that only wild ERdj3 was released by wild type BiP in the presence of ATP, whereas both the QPD and HPN mutants remained bound to the substrate even in the presence of ATP (Figure 4-6C). The combination of this experiment and the previous one (Figure 4-5A) demonstrate that both a $\mathrm{BiP} / \mathrm{ATP} /$ substrate interaction and a functional $\mathrm{BiP} / \mathrm{ERdj} 3$ interaction are required to release ERdj3 from substrate.

In a search for allele specific suppressors of DnaJ HPN and QPD mutants, no suppressors were found for the QPD mutant, but three different DnaK mutants were identified that restored growth at temperatures that were non-permissive for the HPN mutant (Suh et al., 1998). Of these, the DnaK R167H mutant (analogous to our R197H mutant) bound better than wild-type DnaK to the DnaJ HPN mutant. Thus, we wished to determine if our HPN ERdj3 mutant and our R197H BiP mutant would constitute a functional pair that could rescue the inability of HPN ERdj3 to be released from substrate. To determine this, we first measured the ability of both the QPD and HPN mutants to bind to and stimulate the ATPase activity of R197H BiP, which cannot be stimulated with wild-type ERdj3 (Awad et al., 2008). Wild-type and mutant ERdj3 proteins were co-expressed in COS cells with R197H BiP as described above. We found that the HPN mutant was as defective in binding to R197H BiP mutant as either wildtype ERdj3 or the QPD mutant (Figure 4-7A). Thus, unfortunately the HPN ERdj3 mutant did not appear to re-establish a functional pair with the R197H BiP mutant by this criterion. We also examined the ability of the various ERdj3 proteins to stimulate the ATPase activity of R197H BiP, and found that only the HPN mutant showed a very modest ability to stimulate the ATPase activity of R197H BiP (Figure 4-7B). However, this weak stimulation is not comparable to the stimulation of WT ERdj3/ BiP pair. This result is consistent with our in vivo binding data showing that HPN ERdj3 did not bind detectably to R197H BiP, and suggested that the HPN ERdj3/R197H BiP pair did not reform a functional pair as observed for the corresponding mutants in DnaJ and DnaK.

However, since two of the DnaK allele-specific suppressors of the HPN DnaJ mutant did not bind better to HPN DnaJ than wild-type DnaK (Suh et al., 1998), we proceeded to determine if our R197H BiP mutant could release the HPN ERdj3 mutant from denatured luciferase. We found that $\mathrm{R} 197 \mathrm{H} \mathrm{BiP}$ was unable to release either the wild type or the QPD ERdj3 mutant (Figure 4-7C), which is in keeping with the fact that no allele specific suppressors were found for the DnaJ QPD mutant (Suh et al., 1998). However, when we examined the interaction between the R197H BiP mutant and the HPN ERdj3 mutant we observed a very small but reproducible release of the HPN ERdj3 mutant. The amount of release was greater than that observed when wild-type BiP was used to release the HPN mutant but nowhere near that achieved when the wild-type form of each protein was used. This suggests that the $\mathrm{R} 197 \mathrm{H} \mathrm{BiP} / \mathrm{HPN}$ ERdj3 proteins do not constitute a fully functional pair but do possess a small amount of activity together. 
A.

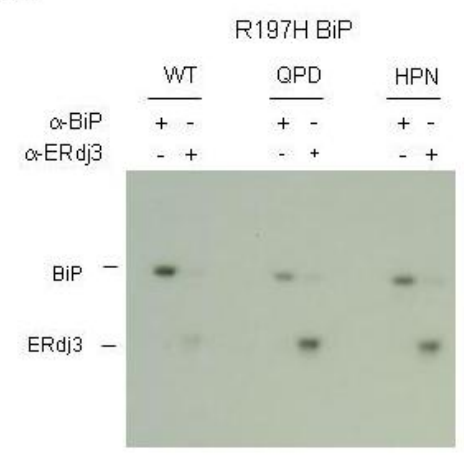

B.

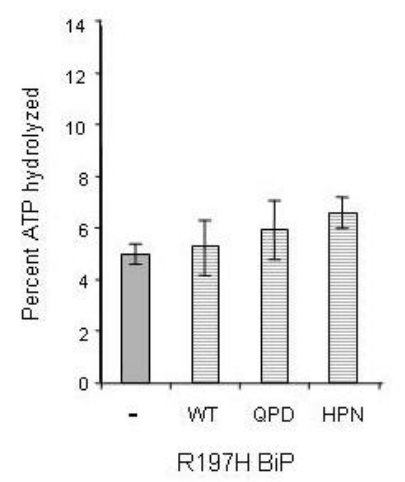

C.

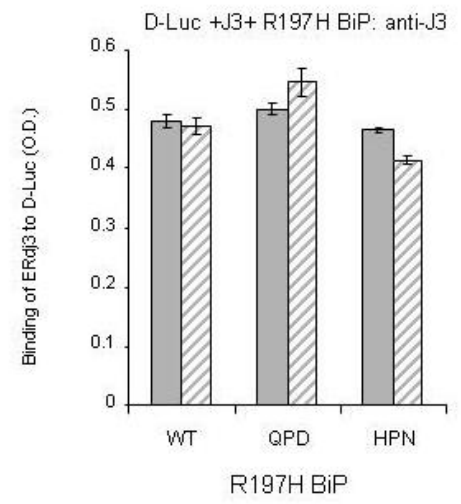

Figure 4-7. R197H BiP cannot be released from D-Luc by either wild-type ERdj3 or the QPD mutant

The experiments were performed exactly the same as in Figure 4-6 except that the R197H BiP mutant was used to release the various ERdj3 proteins. 


\subsection{Discussion}

The vast group of proteins that comprise the DnaJ-like family has been subdivided into three subclasses (Cheetham et al., 1998). The type I and type II proteins bind a broad spectrum of substrates, whereas the type III protein, which possess only a J domain that can be found anywhere in the protein, either are not known to interact directly with substrates or in some cases bind to a much more restricted subset of proteins. There is now a significant amount of data to argue that DnaJ proteins bind to unfolded proteins initially and due to their ability to interact specifically with the ATP form of Hsp70s serve to recruit the open form of the Hsp70 to the substrate. Hsp70 proteins must be able to interact with a DnaJ protein as well as to bind and hydrolyze ATP in order to be efficiently recruited to the substrate (Wawrzynow et al., 1995). Data showing that DnaJ proteins can interact with the SBD of the Hsp70 as well as with its NBD have led investigators to speculate that this might allow the DnaJ protein to deliver and transfer the substrate to the correct region of the Hsp70 protein (Laufen et al., 1999). Several models have been proposed as to how this may occur. First, it is possible that once the DnaJ protein contacts an Hsp70, it releases the unfolded protein and the Hsp70 captures it (Rudiger et al., 2001). The identification of stable DnaJ/Hsp70/substrate complexes (Szabo et al., 1994; Wawrzynow et al., 1995; Han \& Christen, 2003; Shen et al., 2005;) makes this scenario less likely. Second, it has been proposed that the two proteins bind distinct sequences on the substrate, which is supported by the identification of binding sites on substrate proteins for DnaK and DnaJ using peptide libraries (Kim et al., 2002; Han et al., 2003). Once the J domain interacts with the NBD of the Hsp70, it has been argued that this would reorient the Hsp70 and in some way wrench the substrate from the DnaJ protein (Landry, 2003). Our demonstration that wild-type BiP, which binds the substrate but cannot release the QPD or HPN mutant could be interpreted as being compatible with this model, since the absence of the BiP/ERdj3 interaction might not allow BiP to wrench the substrate from ERdj3. This wrenching would need to happen before ATP is hydrolyzed, as DnaJ domains lose affinity for the ADP bound form of Hsp70 (Wawrzynow \& Zylicz, 1995) and maximal stimulation of the ATPase activity of Hsp70s by DnaJs only occurs after the substrate binds to the Hsp70 (Laufen et al., 1999). Finally, it is possible that in addition to the conformational change that DnaJ induces in the Hsp70 protein, that after substrate binding and ATP hydrolysis the Hsp70 protein causes a reciprocal conformational change in the substrate binding domain of the DnaJ protein. It is known that DnaJ proteins have a second interaction site with the SBD of Hsp70s (Laufen et al., 1999), but it is not clear which domain of DnaJ is involved it this binding (Wall et al., 1995; Wawrzynow et al., 1995; Linke et al., 2003; Sahi \& Craig, 2007; nor how alterations in these interactions affect the structure of DnaJ proteins. Our data is also compatible with this model.

Our discovery that the release of ERdj3 from substrate is dependent on a functional interaction with BiP is likely to be true of other DnaJ/Hsp70 pairs. Recent data from Petrova et al., demonstrate a similar requirement for the release of another ER localized DnaJ protein, P58, from substrate. In this study P58 was shown to bind directly to misfolded RNase A in vitro and was released from it by BiP in the presence of ATP and $\mathrm{Mg}^{2+}$. Mutations in either P58 or BiP that disrupted the interaction between them blocked the release of P58 from misfolded RNase A. In addition to interactions between 
the nucleotide binding domain of BiP and the ER localized DnaJ protein, this study further revealed that $\mathrm{BiP}$ also needed to bind to the substrate to trigger DnaJ release. Based on the conservation of interactions between other DnaJ/Hsp70 proteins, we believe this is likely to be a universal requirement for the release of DnaJs. In support of this, an earlier in vitro study found that DnaJ bound to a substrate and prevented its aggregation, but it could not fold the protein unless DnaK and ATP were present (Lu \& Cyr, 1998; Fan et al. 2005). In view of our data, it is reasonable to suggest that DnaJ remained bound to the substrate in the absence of DnaK, thereby preventing its aggregation, but also preventing it from folding. Only in the presence of ATP would both chaperones be released allowing the substrate to fold. We would speculate, based on our data, that in the absence of ATP that both DnaK and DnaJ might bind, but folding would not occur.

Our previous characterization of the interaction of ERdj3 with substrates in cultured cell lines revealed that wild-type ERdj3 disappeared from BiP:substrate complexes long before folding was complete, whereas a QPD mutant remained associated with the substrate (Shen et al., 2005). This led us to speculate that the prolonged binding of the QPD mutant to substrates could be due to its inability to recognize that $\mathrm{BiP}$ had bound productively to the substrate. The data presented here confirm that the release of ERdj3 from substrate, at least in vitro, required an interaction between the $\mathrm{J}$ domain of ERdj3 and NBD of BiP and argue that indeed this is the reason for prolonged association of mutant ERdj3 with substrates in vivo. The in vitro release of ERdj3 from substrate also required BiP's ATPase and substrate binding activity, as neither the T37G, G227D (this study), nor the NBD alone (Petrova et al., 2008) were able to release the corresponding DnaJ proteins from their substrates. These requirements would ensure that once a DnaJ protein engages an unfolded substrate, it would remain bound to prevent aggregation until it had recruited an open form of Hsp70 to the substrate, allowed the Hsp70 to initially associate with the substrate and then hydrolyze ATP to form a more stable interaction. Only when all of these steps had occurred would the DnaJ protein release from the Hsp70 and from the substrate. This scenario is consistent with most published data showing that DnaJ proteins bind first and more transiently than Hsp70, which remain associated until folding is complete.

In summary, our studies show that BiP promotes the release of ERdj3 from substrates in the presence of ATP. This is not due to a competition for binding sites on the substrate once $\mathrm{BiP}$ is recruited through its association with ERdj3, but rather it requires a functional interaction between ERdj3 and BiP. This includes both the ability to physically interact with each other as well as the ability of ERdj3 to stimulate the ATPase activity of BiP. The fact that similar data was observed with another ERdj/BiP pair (Petrova et al., 2008) argues that this mechanism is likely to be used for the release of other DnaJ proteins from substrates. 


\section{CHAPTER 5.DISCUSSION}

Many DnaJ proteins can interact directly with unfolded substrates and prevent them from aggregating in vitro. Crystallographic studies of the $\mathrm{C}$-terminal peptide binding fragments of Ydj1 (Li et al., 2003) and Sis1 (Sha et al., 2000) determined the putative substrate binding of domain of DnaJ proteins. The substrate binding characteristics of mammalian ER DnaJ proteins were poorly understood when I started my project. Since ERdj3 interacts with unassembled Ig heavy chains unusually stably in vivo, it provided a good model to study the interaction between a mammalian DnaJ protein and an unfolded substrate. In my first study (Chapter 2), we characterized the structural requirements for ERdj3 to bind to substrates by using a combination of in vivo and in vitro assays. Our data indicates that hydrophobic amino acids in domain I of ERdj3 are conserved with those of Ydj1 and form a putative substrate binding site. Comparison the structures of domain I of Ydj1 and Sis1 (Sha et al., 2000) suggests that Sis1 is likely to contain the same substrate binding site, even though Sis 1 was crystallized without a bound peptide. Together these studies, coupled with mine, suggest that this substrate binding structure may be common to all type I and II DnaJ proteins. Very recently a structure was solved for the putative peptide-binding fragment of Hdj1(Hu et al., 2008), a human type II DnaJ protein. Like Sis1, this fragment was crystallized without a bound peptide, forms dimers, and has a very similar structure to Ydj1, except that it lacks a domain II.

However, our data revealed that domain II of ERdj3 is also involved in substrate binding, which is not supported by the Ydj1 structure where the peptide only associated with domain I. Since Ydj1 was co-crystallized with a small peptide (GWLYEIS), which is much smaller than natural substrates, it is possible that domain II cooperates with domain I to form a larger substrate binding site in cells allowing Ydj1 to interact with larger polypeptides (Figure 5-1A). The other possibility is that domain II stabilizes the structure of domain I in the absence of substrate (Figure 5-1B), which is supported by the fact that Ydj1's structure could only be solved in the presence of peptide. To test this possibility, we attempted to co-express domain II of ERdj3 (PRSF-II) along with Histagged ERdj3- $\Delta \mathrm{II}$ in bacteria cells and use $\mathrm{Ni}^{2+}$-agarose columns to pull down the induced proteins. If our hypothesis was correct, we expected that domain II would bind to the ERdj3- $\Delta$ II protein in trans and restore the ability of ERdj3- $\Delta$ II to bind to denatured luciferase in vitro. Unfortunately, domain II is so small (about 40 amino acids) that it was not expressed at detectable levels. It might be possible to solve this problem by adding a GST tag to domain II to make a larger protein which might allow it to be expressed more stably in bacteria. However, the GST tag may affect the ability of domain II to interact with domain I and could be removed. This could be done by inserting a cleavage site such as thrombin or factor Xa between the GST tag and domain II. An alternative way to test whether domain II interacts with domain I to stabilize it would be to synthesize the domain II polypeptide in vitro. This strategy may be possible since domain II only has 40 amino acids, which is within the range of what can be synthesized in vitro. By adding domain II back to our binding assay with ERdj3- $\Delta$ II, we could examine its ability to restore ERdj3- $\Delta$ II binding to D-Luc. 
A

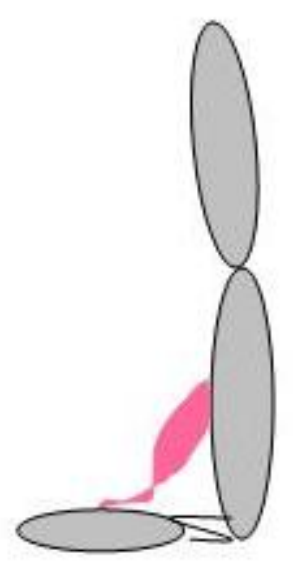

B

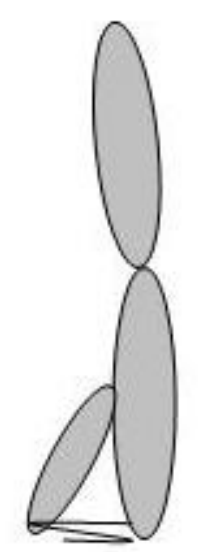

Figure 5-1. Potential roles of domain II is ERdj3 structure

(A) cooperate with domain II to bind to substrate (B) stabilize domain I in the absence of substrate. 
NMR analyses might provide another tool to monitor the possibility that domain II interacts with domain I in the absence of peptide to stabilize this domain. Since a structure has been solved for domains I, II, and III of Ydj1 coupled with peptide, the NMR study of Ydj1 instead of ERdj3 may be a more straightforward way to answer this question. The size of Ydj1's domain I is 79 amino acids and with domain II it is 144 amino acids, which is well within the range for NMR studies. Comparing the structures of a Ydj1 fragment that includes only domain I and II in both the presence and absence of peptide could give an answer. If domain II binds to domain I in the absence of peptide, we would expect to see a change in the substrate binding face at domain I when peptide was added. If domain II serves to provide a larger binding site for different substrates, we would expect to see no change in the substrate binding face in the presence or absence of peptide.

It is important to note that type II DnaJ proteins (such as Sis1 and Hdj1), which lack domain II, can bind to substrates just fine. It has been proposed recently, that domain I of type II DnaJ proteins may possess significant flexibility (Hu et al., 2008), which would allow them to adjust the size or shape of the substrate binding cleft. It is possible that type I proteins achieve this additional flexibility through interactions between domain I and II. It is also very likely that type I and II DnaJ proteins have different substrate specificities that require different structures. However, complementation studies with different DnaJ proteins argue that in most cases these substrates must but not be essential ones.

Although we decided that overall ERdj3 was structurally very similar to Ydj1, the four cysteine residues in domain II of ERdj3 form intra-domain disulfide bonds, whereas the eight cysteines in Ydj1 form two zinc binding sites. Since Ydj1 and ERdj3 belong to different organisms and exist in different organelles, it was expected that they might not be interchangeable. Ydj1 expressed in the ER would not bind zinc atoms, and ERdj3 expressed in the cytosol was unlikely to form disulfide bonds. This led us to speculate that verification of the role of domain II of ERdj3 in substrate interaction would be possible by creating chimera proteins made up of various combinations of ERdj3 and Ydj1 domains. The fact that ER expressed Ydj1 can interact with BiP and ERdj3 substrate (Figure 4-2) denied that strategy but led to studies described in Chapter 3. In this study, we found that ERdj3 could compensate the loss of Ydj1 and that both the ability to stimulate the ATPase activity of the Hsp70 partner and to bind to substrates were necessary for ERdj 3 to compensate for the loss of Ydj1. Because domain II seems to be important for ERdj3's substrate binding function and the intra-domain disulfide bonds were reported to be critical for substrate binding in vitro, we discussed the three possibilities in Chapter 3 to account for this. First, we argued that it is possible that the disulfide bonds do form in ERdj3 even in the relatively reducing environment of the cytosol. Second, these cysteines may be in a reduced state in the yeast cytosol, but the formation of disulfide bonds might not be necessary for ERdj3's function in vivo. Finally, we suggested that when ERdj 3 is expressed in the yeast cytosol, it is possible that the cysteines in domain II of ERdj3 could bind to $\mathrm{Zn}^{2+}$, which would serve to bridge the four cysteines and might stabilize this domain by mimicking the conformational stability that disulfide bonds provide. To verify whether ERdj3 forms disulfide bonds in the yeast 
cytosol, we could express ERdj3 in yeast cells, isolate the protein and then subject it to reducing and nonreducing SDS-PAGE to check its migration (Figure 5-2). If disulfide bonds form in the cytosol, we will find that ERdj3 migrates slower in reducing SDSPAGE than under non-reducing conditions (pattern \#1). If there is no change in the migration of the protein with the addition of reducing agents, it would mean either that disulfide bonds do not form (pattern \#2) or that zinc is binding to the cysteines and is not removed by reducing agents in the sample buffer (pattern \#3). To distinguish between the second and third possibilities, we could compare their mobility to that of reduced and non-reduced ERdj3 isolated from mammalian cells. If the ERdj3 expressed in yeast migrates with the reduced form of mammalian ERdj3, it would argue that no disulfide bonds form, whereas if it migrates faster than the reduced form, it might suggest that zinc is binding to these cysteines and holding domain II in a more compact form. To test this possibility, we could treat the ERdj3 isolated from the yeast cytosol with $\rho$ hydroxymercuripherysulfon which is sulfhydryl-dissociating reagent and was used to remove Zinc from DnaJ (Tang et al., 2001).

Although it is fairly well understood how Hsp70 proteins are released from substrates, very little is known about how DnaJ proteins are released. Our in vivo data showing that ERdj3 proteins that could not interact with BiP actually resulted in more ERdj3 being associated with the substrate (Chapter 2) led us to speculate that this might occur due to decreased release of ERdj3 from substrate and not due to increased binding. We hypothesized that a functional interaction between DnaJs and Hsp70s might be required to release DnaJ from substrates. The development of an in vitro binding assay (Chapter 2) allowed me to test this. Using a series of ERdj3 and BiP mutants with denatured luciferase as the substrate, we determined that release of ERdj3 required the ATPase activity of BiP and the ability of ERdj3 and BiP to interact. To demonstrate the latter point, we used BiP mutants that could not bind to ERdj3 as well as ERdj3 mutants that could not bind to BiP. We suggested that the interaction with BiP may induce a reciprocal change in ERdj3 that triggers its release from substrates (Chapter 4). This allowed us to propose a general model for the release of DnaJ proteins from substrates (Figure 5-3). However, we did not demonstrate that ERdj3 actually undergoes a conformational change in that study. Protease sensitivity is a method that is often used to measure conformational changes. It is tricky for our assay, since we would need to retrieve ERdj3 protein bound to substrate and BiP and compare it to released ERdj3. We would expect there to be changes in protease sensitivity due to substrate binding that could not be easily distinguished from conformational changes in ERdj3 itself. Conformational changes can also be monitored by measuring changes in tryptophan fluorescence. This assay has been used to monitor conformational changes in Hsp70 proteins that occur in response to nucleotide binding. This type of assay is dependent on the local environment of the tryptophan changing in response to a larger conformational change in the protein. It is possible that this could be an alternative way to answer the question. Among the three proteins in our reaction, there are no tryptophan residues in luciferase, two in BiP (which would have to be mutated), and only one in ERdj3, which occurs in domain III. If no change is observed, we would probably need to engineer another tryptophan into another region of ERdj3, although it is not clear where the best 


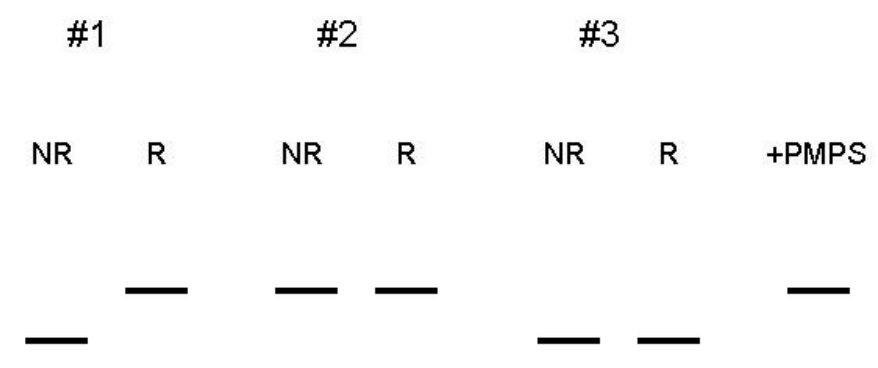

Figure 5-2. Potential patterns of cytosolic ERdj3 


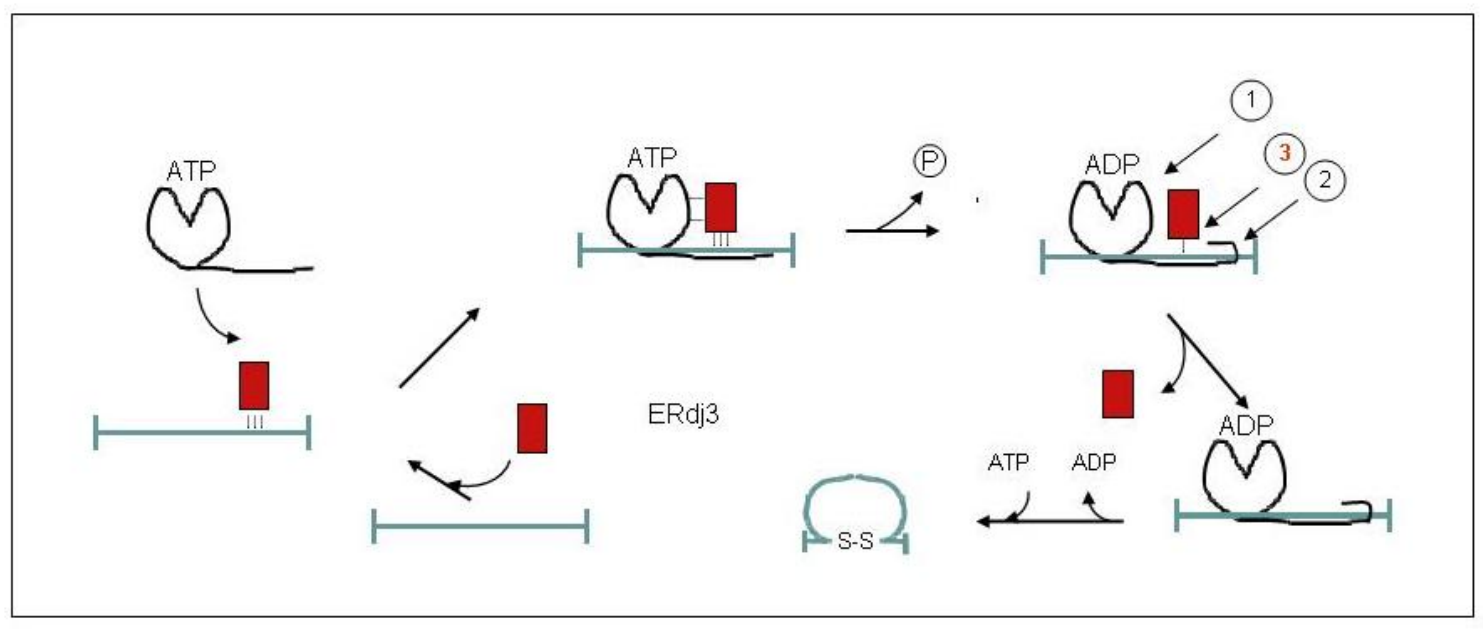

Figure 5-3. Model for dissociation of ERdj3 from substrate proteins

ERdj3 binds directly to substrates and recruits the ATP bound form of BiP. A transient 3-way complex is formed. ERdj3 activates the ATPase activity of BiP, which decreases the affinity of ERdj3 for BiP (step 1) and induces a conformational change in the substrate binding domain of BiP, which stabilizes its binding substrate (step 2). We hypothesis that this interaction triggers a reciprocal conformational change in ERdj3 that decreases its affinity for substrate (step 3), allowing it to dissociate. 
place to put the tryptophan would be. Alternatively, it might be necessary to switch to another DnaJ protein.

A separate limitation of our study (Chapter 4) was that all of our BiP ATPase mutants were also defective in binding to ERdj3, which made it impossible to separate which defect led to an inability to release ERdj3 from substrate. A recent paper reported that the E175S Hsc70 mutant has a very low ATPase activity but is still able to bind to DnaJ proteins (Jiang et al., 2007). Our lab had previously produced the corresponding point mutation in hamster BiP (E201G) and reported that it has very low ATPase activity, but still can bind ATP and substrate (Gaut \& Hendershot, 1993). If this BiP mutant behaves the same as the E175S Hsc70 mutant and is able to interact with ERdj3, we could use it in our release assay to examine whether both the ATPase activity and binding to ERdj3 are required or if only the binding to ERdj3 is sufficient for its release.

In conclusion, our studies characterized the structural requirements for ERdj3's ability to bind to substrates and further determined the requirements for producing functional Hsp70-DnaJ pairs. In addition, my studies provided further insights on the mechanism used to release ERdj3 from substrates. The fact that Hsp70s and DnaJ proteins are so well conserved suggests that our findings have general implications for Hsp70-DnaJ pairs. Hopefully our data will encourage others to further explore these possibilities. 


\section{LIST OF REFERENCES}

1. Alder NN, Shen Y, Brodsky JL, Hendershot LM, and Johnson AE (2005) The molecular mechanisms underlying BiP-mediated gating of the Sec61 translocon of the endoplasmic reticulum. $J$ Cell Biol, 168, 389-399.

2. Anfinsen CB (1972) The formation and stabilization of protein structure. Biochem $J, 128,737-749$.

3. Anttonen AK, Siintola E, Tranebjaerg L, Iwata NK, Bijlsma EK, Meguro H, Ichikawa Y, Goto J, Kopra O, and Lehesjoki AE (2008) Novel SIL1 mutations and exclusion of functional candidate genes in Marinesco-Sjogren syndrome. Eur J Hum Genet, 16, 961-969.

4. Awad W, Estrada I, Shen Y, and Hendershot LM (2008) BiP mutants that are unable to interact with endoplasmic reticulum DnaJ proteins provide insights into interdomain interactions in BiP. Proc Natl Acad Sci U S A, 105, 1164-1169.

5. Banecki B, Liberek K, Wall D, Wawrzynow A, Georgopoulos C, Bertoli E, Tanfani F, and Zylicz M (1996) Structure-function analysis of the zinc finger region of the DnaJ molecular chaperone. J Biol Chem, 271, 14840-14848.

6. Bardwell JC, Tilly K, Craig E, King J, Zylicz M, and Georgopoulos C (1986) The nucleotide sequence of the Escherichia coli K12 dnaJ+ gene. A gene that encodes a heat shock protein. $J$ Biol Chem, 261, 1782-1785.

$7 \quad$ Bergman LW and Kuehl WM (1979) Formation of an intrachain disulfide bond on nascent immunoglobulin light chains. J Biol Chem, 254, 8869-8876.

8. Bertolotti A, Zhang Y, Hendershot LM, Harding HP, and Ron D (2000) Dynamic interaction of $\mathrm{BiP}$ and $\mathrm{ER}$ stress transducers in the unfolded-protein response. Nat Cell Biol, 2, 326-332.

9. Blond-Elguindi S, Cwirla SE, Dower WJ, Lipshutz RJ, Sprang SR, Sambrook JF, and Gething MJ (1993) Affinity panning of a library of peptides displayed on bacteriophages reveals the binding specificity of BiP. Cell, 75, 717-728.

11. Boisrame A, Kabani M, Beckerich JM, Hartmann E, and Gaillardin C (1998) Interaction of Kar2p and Sls1p is required for efficient co-translational translocation of secreted proteins in the yeast Yarrowia lipolytica. J Biol Chem, 273, 30903-30908.

12. Bole DG, Hendershot LM, and Kearney JF (1986) Posttranslational association of immunoglobulin heavy chain binding protein with nascent heavy chains in nonsecreting and secreting hybridomas. J Cell Biol, 102, 1558-1566. 
13. Bolliger L, Deloche O, Glick BS, Georgopoulos C, Jeno P, Kronidou N, Horst M, Morishima N, and Schatz G (1994) A mitochondrial homolog of bacterial GrpE interacts with mitochondrial hsp70 and is essential for viability. EMBO J, 13, 1998-2006.

14. Brightman SE, Blatch GL, and Zetter BR (1995) Isolation of a mouse cDNA encoding MTJ1, a new murine member of the DnaJ family of proteins. Gene, 153, 249-254.

15. Brodsky JL, Goeckeler J, and Schekman R (1995) BiP and Sec63p are required for both co- and posttranslational protein translocation into the yeast endoplasmic reticulum. Proc Natl Acad Sci U S A, 92, 9643-9646.

16. Buchberger A, Theyssen H, Schroder H, McCarty JS, Virgallita G, Milkereit P, Reinstein J, and Bukau B (1995) Nucleotide-induced conformational changes in the ATPase and substrate binding domains of the DnaK chaperone provide evidence for interdomain communication. J Biol Chem, 270, 16903-16910.

17. Caplan AJ and Douglas MG (1991) Characterization of YDJ1: a yeast homologue of the bacterial dnaJ protein. $J$ Cell Biol, 114, 609-621.

18. Caplan AJ, Cyr DM, and Douglas MG (1992) YDJ1p facilitates polypeptide translocation across different intracellular membranes by a conserved mechanism. Cell, 71, 1143-1155.

19. Caplan AJ, Cyr DM, and Douglas MG (1993) Eukaryotic homologues of Escherichia coli dnaJ: a diverse protein family that functions with hsp70 stress proteins. Mol Biol Cell, 4, 555-563.

20. Caplan AJ, Tsai J, Casey PJ, and Douglas MG (1992b) Farnesylation of YDJ1p is required for function at elevated growth temperatures in Saccharomyces cerevisiae. J Biol Chem, 267, 18890-18895.

21. Cheetham ME and Caplan AJ (1998) Structure, function and evolution of DnaJ: conservation and adaptation of chaperone function. Cell Stress Chaperones, 3, 28 36.

22. Chen W, Helenius J, Braakman I, and Helenius A (1995) Cotranslational folding and calnexin binding during glycoprotein synthesis. Proc Natl Acad Sci US A, 92, 6229-6233.

23. Chevalier M, King L, and Blond S (1998) Purification and properties of BiP. Methods Enzymol, 290, 384-409.

24. Chevalier M, King L, Wang C, Gething MJ, Elguindi E, and Blond SY (1998) Substrate binding induces depolymerization of the C-terminal peptide binding domain of murine GRP78/BiP. J Biol Chem, 273, 26827-26835. 
25. Chevalier M, Rhee H, Elguindi EC, and Blond SY (2000) Interaction of murine BiP/GRP78 with the DnaJ homologue MTJ1.J Biol Chem, 275, 19620-19627.

26. Chung KT, Shen Y, and Hendershot LM (2002) BAP, a mammalian BiP associated protein, is a nucleotide exchange factor that regulates the ATPase activity of BiP. J Biol Chem, 277, 47557-47563.

27. Corsi AK and Schekman R (1997) The lumenal domain of Sec63p stimulates the ATPase activity of BiP and mediates $\mathrm{BiP}$ recruitment to the translocon in Saccharomyces cerevisiae. J Cell Biol, 137, 1483-1493.

28. Cox JS, Walter P (1996) A novel mechanism for regulating activity of a transcription factor that controls the unfolded protein response. Cell. 87(3):391404.

29. Craven RA, Egerton M, and Stirling CJ (1996) A novel Hsp70 of the yeast ER lumen is required for the efficient translocation of a number of protein precursors. EMBO J, 15, 2640-2650.

30. Cunnea PM, Miranda-Vizuete A, Bertoli G, Simmen T, Damdimopoulos AE, Hermann S, Leinonen S, Huikko MP, Gustafsson JA, Sitia R, and Spyrou G (2003) ERdj5, an endoplasmic reticulum (ER)-resident protein containing DnaJ and thioredoxin domains, is expressed in secretory cells or following ER stress. $J$ Biol Chem, 278, 1059-1066.

31. Cyr DM, Lu X, Douglas MG. (1992) Regulation of Hsp70 function by a eukaryotic DnaJ homolog. J Biol Chem, 267, 20927-20931.

32. DeCaprio JA (1999) The role of the J domain of SV40 large T in cellular transformation. Biologicals, 27, 23-28.

33. Dierks T, Volkmer J, Schlenstedt G, Jung C, Sandholzer U, Zachmann K, Schlotterhose P, Neifer K, Schmidt B, and Zimmermann R (1996) A microsomal ATP-binding protein involved in efficient protein transport into the mammalian endoplasmic reticulum. EMBO J, 15, 6931-6942.

34. Dong M, Bridges JP, Apsley K, Xu Y, and Weaver TE (2008) ERdj4 and ERdj5 Are Required for Endoplasmic Reticulum-associated Protein Degradation of Misfolded Surfactant Protein C. Mol Biol Cell, 19, 2620-2630.

35. Dudek J, Volkmer J, Bies C, Guth S, Muller A, Lerner M, Feick P, Schafer KH, Morgenstern E, Hennessy F, Blatch GL, Janoscheck K, Heim N, Scholtes P, Frien M, Nastainczyk W, and Zimmermann R (2002) A novel type of co-chaperone mediates transmembrane recruitment of DnaK-like chaperones to ribosomes. EMBO J, 21, 2958-2967.

36. Ellgaard L and Helenius A (2001) ER quality control: towards an understanding at the molecular level. Curr Opin Cell Biol, 13, 431-437. 
37. Ellgaard L, Molinari M, and Helenius A (1999) Setting the standards: quality control in the secretory pathway. Science, 286, 1882-1888.

38. Ellis J (1987) Proteins as molecular chaperones. Nature, 328, 378-379.

39. Erbse A, Mayer MP, and Bukau B (2004) Mechanism of substrate recognition by Hsp70 chaperones. Biochem Soc Trans, 32, 617-621.

40. Fan CY, Lee S, and Cyr DM (2003) Mechanisms for regulation of Hsp70 function by Hsp40. Cell Stress Chaperones, 8, 309-316.

41. Flynn GC, Chappell TG, and Rothman JE (1989) Peptide binding and release by proteins implicated as catalysts of protein assembly. Science, 245, 385-390.

42. Flynn GC, Pohl J, Flocco MT, and Rothman JE (1991) Peptide-binding specificity of the molecular chaperone BiP. Nature, 353, 726-730.

43. Fourie AM, Sambrook JF, and Gething MJ (1994) Common and divergent peptide binding specificities of hsp70 molecular chaperones. J Biol Chem, 269, 30470-30478.

44. Freeman BC, Myers MP, Schumacher R, and Morimoto RI (1995) Identification of a regulatory motif in Hsp70 that affects ATPase activity, substrate binding and interaction with HDJ-1. EMBO J, 14, 2281-2292.

45. Frydman J, Nimmesgern E, Erdjument-Bromage H, Wall JS, Tempst P, and Hartl FU (1992) Function in protein folding of TRiC, a cytosolic ring complex containing TCP-1 and structurally related subunits. EMBO J, 11, 4767-4778.

46. Gassler CS, Buchberger A, Laufen T, Mayer MP, Schroder H, Valencia A, and Bukau B (1998) Mutations in the DnaK chaperone affecting interaction with the DnaJ cochaperone. Proc Natl Acad Sci U S A, 95, 15229-15234.

47. Gaut JR and Hendershot LM (1993) Mutations within the nucleotide binding site of immunoglobulin-binding protein inhibit ATPase activity and interfere with release of immunoglobulin heavy chain. $J$ Biol Chem, 268, 7248-7255.

48. Gragerov A and Gottesman ME (1994) Different peptide binding specificities of hsp70 family members. $J$ Mol Biol, 241, 133-135.

49. Gruschus JM, Han CJ, Greener T, Ferretti JA, Greene LE, and Eisenberg E (2004) Structure of the functional fragment of auxilin required for catalytic uncoating of clathrin-coated vesicles. Biochemistry, 43, 3111-3119.

50. Haas IG and Meo T (1988) cDNA cloning of the immunoglobulin heavy chain binding protein. Proc Natl Acad Sci U S A, 85, 2250-2254. 
51. Haas IG and Wabl M (1983) Immunoglobulin heavy chain binding protein. Nature, 306, 387-389.

52. Hamman BD, Hendershot LM, and Johnson AE (1998) BiP maintains the permeability barrier of the ER membrane by sealing the lumenal end of the translocon pore before and early in translocation. Cell, 92, 747-758.

53. Hammond C and Helenius A (1995) Quality control in the secretory pathway. Curr Opin Cell Biol, 7, 523-529.

54. Hampton RY (2002) ER-associated degradation in protein quality control and cellular regulation. Curr Opin Cell Biol, 14, 476-482.

55. Han W and Christen P (2003) Mechanism of the targeting action of DnaJ in the DnaK molecular chaperone system. J Biol Chem, 278, 19038-19043.

56. Harding HP, Zhang Y, and Ron D (1999) Protein translation and folding are coupled by an endoplasmic-reticulum-resident kinase. Nature, 397, 271-274.

57. Harrison CJ, Hayer-Hartl M, Di LM, Hartl F, and Kuriyan J (1997) Crystal structure of the nucleotide exchange factor GrpE bound to the ATPase domain of the molecular chaperone DnaK. Science, 276, 431-435.

58. Hartl FU (1991) Heat shock proteins in protein folding and membrane translocation. Semin Immunol, 3, 5-16.

59. Hayer-Hartl MK, Ewbank JJ, Creighton TE, and Hartl FU (1994) Conformational specificity of the chaperonin GroEL for the compact folding intermediates of alpha-lactalbumin. EMBO J, 13, 3192-3202.

60. Haze K, Yoshida H, Yanagi H, Yura T, and Mori K (1999) Mammalian transcription factor ATF6 is synthesized as a transmembrane protein and activated by proteolysis in response to endoplasmic reticulum stress. Mol Biol Cell, 10, 3787-3799.

61. Hellman R, Vanhove M, Lejeune A, Stevens FJ, Hendershot LM (1999) The in vivo association of BiP with newly synthesized proteins is dependent on the rate and stability of folding and not simply on the presence of sequences that can bind to BiP. J Cell Biol, 144(1):21-30.

62. Hemmingsen SM, Woolford C, van d, V, Tilly K, Dennis DT, Georgopoulos CP, Hendrix RW, and Ellis RJ (1988) Homologous plant and bacterial proteins chaperone oligomeric protein assembly. Nature, 333, 330-334.

63. Hendrick JP, Langer T, Davis TA, Hartl FU, and Wiedmann M (1993) Control of folding and membrane translocation by binding of the chaperone DnaJ to nascent polypeptides. Proc Natl Acad Sci U S A, 90, 10216-10220. 
64. Hennessy F, Cheetham ME, Dirr HW, and Blatch GL (2000) Analysis of the levels of conservation of the J domain among the various types of DnaJ-like proteins. Cell Stress Chaperones, 5, 347-358.

65. Hohfeld J and Jentsch S (1997) GrpE-like regulation of the hsc 70 chaperone by the anti-apoptotic protein BAG-1. EMBO J, 16, 6209-6216.

66. Hohfeld J, Minami Y, and Hartl FU (1995) Hip, a novel cochaperone involved in the eukaryotic Hsc70/Hsp40 reaction cycle. Cell, 83, 589-598.

67. Hosoda A, Kimata Y, Tsuru A, and Kohno K (2003) JPDI, a novel endoplasmic reticulum-resident protein containing both a BiP-interacting J-domain and thioredoxin-like motifs. J Biol Chem, 278, 2669-2676.

68. Hu J, Wu Y, Li J, Qian X, Fu Z, and Sha B (2008) The crystal structure of the putative peptide-binding fragment from the human Hsp40 protein Hdj1. BMC Struct Biol, 8, 3.

69. Hwang C, Sinskey AJ, and Lodish HF (1992) Oxidized redox state of glutathione in the endoplasmic reticulum. Science, 257, 1496-1502.

70. Ito H, Fukuda Y, Murata K, Kimura A (1983) Transformation of intact yeast cells treated with alkali cations. J Bacteriol. 153(1):163-8.

71. Jiang J, Prasad K, Lafer EM, and Sousa R (2005) Structural basis of interdomain communication in the Hsc70 chaperone. Mol Cell, 20, 513-524.

72. Jin Y, Walid A, Hendershot LM (submitted) Regulated release of ERdj3 from unfolded proteins by $\mathrm{BiP}$

73. Johnson JL and Craig EA (2001) An essential role for the substrate-binding region of Hsp40s in Saccharomyces cerevisiae. J Cell Biol, 152, 851-856.

74. Jansens A, Van DE, and Braakman I (2002) Coordinated nonvectorial folding in a newly synthesized multidomain protein. Science, 298, 2401-2403.

75. Jarosch E, Lenk U, and Sommer T (2003) Endoplasmic reticulum-associated protein degradation. Int Rev Cytol, 223, 39-81.

76. Jiang J, Maes EG, Taylor AB, Wang L, Hinck AP, Lafer EM, and Sousa R (2007) Structural basis of J cochaperone binding and regulation of Hsp70. Mol Cell, 28, 422-433.

77. Johannes L and Goud B (2000) Facing inward from compartment shores: how many pathways were we looking for? Traffic, 1, 119-123. 
78. Kabani M, Beckerich JM, and Gaillardin C (2000) Sls1p stimulates Sec63pmediated activation of Kar2p in a conformation-dependent manner in the yeast endoplasmic reticulum. Mol Cell Biol, 20, 6923-6934.

79. Kabani M, Kelley SS, Morrow MW, Montgomery DL, Sivendran R, Rose MD, Gierasch LM, and Brodsky JL (2003) Dependence of endoplasmic reticulumassociated degradation on the peptide binding domain and concentration of BiP. Mol Biol Cell, 14, 3437-3448.

80. Kassenbrock CK and Kelly RB (1989) Interaction of heavy chain binding protein (BiP/GRP78) with adenine nucleotides. EMBO J, 8, 1461-1467.

81. Kelley WL and Georgopoulos C (1997) The $\mathrm{T} / \mathrm{t}$ common exon of simian virus 40, $\mathrm{JC}$, and BK polyomavirus $\mathrm{T}$ antigens can functionally replace the J-domain of the Escherichia coli DnaJ molecular chaperone. Proc Natl Acad Sci U S A, 94, 36793684 .

82. Kelley WL and Landry SJ (1994) Chaperone power in a virus? Trends Biochem Sci, 19, 277-278.

83 Kim SY, Sharma S, Hoskins JR, and Wickner S (2002) Interaction of the DnaK and DnaJ chaperone system with a native substrate, P1 RepA.J Biol Chem, 277, 44778-44783.

84. Laloraya S, Gambill BD, and Craig EA (1994) A role for a eukaryotic GrpErelated protein, Mge1p, in protein translocation. Proc Natl Acad Sci U S A, 91, 6481-6485.

85. Land A, Zonneveld D, and Braakman I (2003) Folding of HIV-1 envelope glycoprotein involves extensive isomerization of disulfide bonds and conformation-dependent leader peptide cleavage. FASEB J, 17, 1058-1067.

86. Landry SJ (2003) Swivels and stators in the Hsp40-Hsp70 chaperone machine. Structure, 11, 1465-1466.

87. Landry SJ, Jordan R, McMacken R, and Gierasch LM (1992) Different conformations for the same polypeptide bound to chaperones DnaK and GroEL. Nature, 355, 455-457.

88. Laskey RA, Honda BM, Mills AD, and Finch JT (1978) Nucleosomes are assembled by an acidic protein which binds histones and transfers them to DNA. Nature, 275, 416-420.

89. Lau PP, Villanueva H, Kobayashi K, Nakamuta M, Chang BH, and Chan L (2001) A DnaJ protein, apobec-1-binding protein-2, modulates apolipoprotein B mRNA editing. $J$ Biol Chem, 276, 46445-46452. 
90. Laufen T, Mayer MP, Beisel C, Klostermeier D, Mogk A, Reinstein J, and Bukau B (1999) Mechanism of regulation of hsp70 chaperones by DnaJ cochaperones. Proc Natl Acad Sci U S A, 96, 5452-5457.

91. Lee AH, Iwakoshi NN, and Glimcher LH (2003) XBP-1 regulates a subset of endoplasmic reticulum resident chaperone genes in the unfolded protein response. Mol Cell Biol, 23, 7448-7459.

92. Lee AS (1987) Coordinated regulation of a set of genes by glucose and calcium ionophores in mammalian cells. Trends Biochem Sci, 12, 20-23.

93. Lee S, Fan CY, Younger JM, Ren H, and Cyr DM (2002) Identification of essential residues in the type II Hsp40 Sis 1 that function in polypeptide binding. $J$ Biol Chem, 277, 21675-21682

94. Lee YK, Brewer JW, Hellman R, and Hendershot LM (1999) BiP and immunoglobulin light chain cooperate to control the folding of heavy chain and ensure the fidelity of immunoglobulin assembly. Mol Biol Cell, 10, 2209-2219.

95. Li J, Qian X, and Sha B (2003) The crystal structure of the yeast Hsp40 Ydj1 complexed with its peptide substrate. Structure, 11, 1475-1483.

96. Li J and Sha B (2005) Structure-based mutagenesis studies of the peptide substrate binding fragment of type I heat-shock protein 40. Biochem J, 386, 453460.

97. Li J, Wu Y, Qian X, and Sha B (2006) Crystal structure of yeast Sis1 peptidebinding fragment and Hsp70 Ssa1 C-terminal complex. Biochem J, 398, 353-360

98. Liberek K, Marszalek J, Ang D, Georgopoulos C, and Zylicz M (1991) Escherichia coli DnaJ and GrpE heat shock proteins jointly stimulate ATPase activity of DnaK. Proc Natl Acad Sci U S A, 88, 2874-2878.

99. Liberek K, Osipiuk J, Zylicz M, Ang D, Skorko J, and Georgopoulos C (1990) Physical interactions between bacteriophage and Escherichia coli proteins required for initiation of lambda DNA replication. J Biol Chem, 265, 3022-3029.

100. Liberek K, Skowyra D, Zylicz M, Johnson C, and Georgopoulos C (1991) The Escherichia coli DnaK chaperone, the $70-\mathrm{kDa}$ heat shock protein eukaryotic equivalent, changes conformation upon ATP hydrolysis, thus triggering its dissociation from a bound target protein. J Biol Chem, 266, 14491-14496.

101. Lievremont JP, Rizzuto R, Hendershot L, and Meldolesi J (1997) BiP, a major chaperone protein of the endoplasmic reticulum lumen, plays a direct and important role in the storage of the rapidly exchanging pool of $\mathrm{Ca} 2+. J \mathrm{Biol}$ Chem, 272, 30873-30879. 
102. Lingappa VR, Katz FN, Lodish HF, and Blobel G (1978) A signal sequence for the insertion of a transmembrane glycoprotein. Similarities to the signals of secretory proteins in primary structure and function. J Biol Chem, 253, 86678670.

103. Linke K, Wolfram T, Bussemer J, and Jakob U (2003) The roles of the two zinc binding sites in DnaJ. J Biol Chem, 278, 44457-44466.

104. Liu Q and Hendrickson WA (2007) Insights into hsp70 chaperone activity from a crystal structure of the yeast Hsp110 Sse1. Cell, 131, 106-120.

105. Lu Z and Cyr DM (1998a) The conserved carboxyl terminus and zinc finger-like domain of the co-chaperone Ydj1 assist Hsp70 in protein folding. $J$ Biol Chem, 273, 5970-5978.

106. $\mathrm{Lu} \mathrm{Z}$ and Cyr DM (1998b) Protein folding activity of Hsp70 is modified differentially by the hsp40 co-chaperones Sis1 and Ydj1.J Biol Chem, 273, 27824-27830.

107. Luke MM, Sutton A, and Arndt KT (1991) Characterization of SIS1, a Saccharomyces cerevisiae homologue of bacterial dnaJ proteins. J Cell Biol, 114, 623-638.

108. Ma Y and Hendershot LM (2004) The role of the unfolded protein response in tumour development: friend or foe? Nat Rev Cancer, 4, 966-977.

109. Marcus NY, Marcus RA, Schmidt BZ, and Haslam DB (2007) Contribution of the HEDJ/ERdj3 cysteine-rich domain to substrate interactions. Arch Biochem Biophys, 468, 147-158.

110. Martin J, Mayhew M, Langer T, and Hartl FU (1993) The reaction cycle of GroEL and GroES in chaperonin-assisted protein folding. Nature, 366, 228-233.

111. Mayer MP, Laufen T, Paal K, McCarty JS, and Bukau B (1999) Investigation of the interaction between DnaK and DnaJ by surface plasmon resonance spectroscopy. J Mol Biol, 289, 1131-1144.

112. McClellan AJ, Endres JB, Vogel JP, Palazzi D, Rose MD, and Brodsky JL (1998) Specific molecular chaperone interactions and an ATP-dependent conformational change are required during posttranslational protein translocation into the yeast ER. Mol Biol Cell.(12):3533-45

113. Melville MW, Hansen WJ, Freeman BC, Welch WJ, and Katze MG (1997) The molecular chaperone hsp40 regulates the activity of P58IPK, the cellular inhibitor of PKR. Proc Natl Acad Sci U S A, 94:97-102. 
114. Meunier L, Usherwood YK, Chung KT, and Hendershot LM (2002) A subset of chaperones and folding enzymes form multiprotein complexes in endoplasmic reticulum to bind nascent proteins. Mol Biol Cell, 13, 4456-4469.

115. Meyer HA, Grau H, Kraft R, Kostka S, Prehn S, Kalies KU, and Hartmann E (2000) Mammalian Sec61 is associated with Sec62 and Sec63. J Biol Chem, 275, 14550-14557.

116. Molinari M, Galli C, Piccaluga V, Pieren M, and Paganetti P (2002) Sequential assistance of molecular chaperones and transient formation of covalent complexes during protein degradation from the ER. $J$ Cell Biol, 158, 247-257.

117. Munro S and Pelham HR (1986) An Hsp70-like protein in the ER: identity with the $78 \mathrm{kd}$ glucose-regulated protein and immunoglobulin heavy chain binding protein. Cell, 46, 291-300.

118. Munro S and Pelham HR (1987) A C-terminal signal prevents secretion of luminal ER proteins. Cell, 48, 899-907.

119. Naylor DJ, Ryan MT, Condron R, Hoogenraad NJ, and Hoj PB (1995) Affinitypurification and identification of GrpE homologues from mammalian mitochondria. Biochim Biophys Acta, 1248, 75-79.

120. Naylor DJ, Stines AP, Hoogenraad NJ, and Hoj PB (1998) Evidence for the existence of distinct mammalian cytosolic, microsomal, and two mitochondrial GrpE-like proteins, the Co-chaperones of specific Hsp70 members. J Biol Chem, 273, 21169-21177.

121. Nishikawa S and Endo T (1997) The yeast JEM1p is a DnaJ-like protein of the endoplasmic reticulum membrane required for nuclear fusion. J Biol Chem, 272, 12889-12892.

122. Nishikawa SI, Fewell SW, Kato Y, Brodsky JL, and Endo T (2001) Molecular chaperones in the yeast endoplasmic reticulum maintain the solubility of proteins for retrotranslocation and degradation. J Cell Biol, 153, 1061-1070.

123. Okazaki A, Ikura T, Nikaido K, and Kuwajima K (1994) The chaperonin GroEL does not recognize apo-alpha-lactalbumin in the molten globule state. Nat Struct Biol, 1, 439-446.

124. Okuda-Shimizu Y and Hendershot LM (2007) Characterization of an ERAD pathway for nonglycosylated BiP substrates, which require Herp. Mol Cell, 28, 544-554.

125. Pace CN, Shirley BA, McNutt M, and Gajiwala K (1996) Forces contributing to the conformational stability of proteins. FASEB J, 10, 75-83. 
126. Peden KW, Srinivasan A, Farber JM (1989) Mutants with changes within or near a hydrophobic region of simian virus 40 large tumor antigen are defective for binding cellular protein p53. Virology 168: 13-21.

127. Petrova K, Oyadomari S, Hendershot L, and Ron D (2008) Regulated association of misfolded endoplamic reticulum lumenal proteins with P58/DNAJc3. submitted.

128. Prapapanich V, Chen S, Toran EJ, Rimerman RA, and Smith DF (1996) Mutational analysis of the hsp70-interacting protein Hip. Mol Cell Biol, 16, 62006207.

129. Prols F, Mayer MP, Renner O, Czarnecki PG, Ast M, Gassler C, Wilting J, Kurz $\mathrm{H}$, and Christ B (2001) Upregulation of the cochaperone Mdg1 in endothelial cells is induced by stress and during in vitro angiogenesis. Exp Cell Res, 269, 4253.

130. Raviol H, Sadlish H, Rodriguez F, Mayer MP, and Bukau B (2006) Chaperone network in the yeast cytosol: Hsp110 is revealed as an Hsp70 nucleotide exchange factor. $E M B O J, 25,2510-2518$.

131. Raynes DA and Guerriero V, Jr. (1998) Inhibition of Hsp70 ATPase activity and protein renaturation by a novel Hsp70-binding protein. J Biol Chem, 273, 3288332888 .

132. Rothblatt JA, Deshaies RJ, Sanders SL, Daum G, and Schekman R (1989) Multiple genes are required for proper insertion of secretory proteins into the endoplasmic reticulum in yeast. $J$ Cell Biol, 109, 2641-2652.

133. Roy B and Lee AS (1995) Transduction of calcium stress through interaction of the human transcription factor $\mathrm{CBF}$ with the proximal CCAAT regulatory element of the grp78/BiP promoter. Mol Cell Biol, 15, 2263-2274.

134. Rudiger S, Germeroth L, Schneider-Mergener J, and Bukau B (1997) Substrate specificity of the DnaK chaperone determined by screening cellulose-bound peptide libraries. EMBOJ, 16, 1501-1507.

135. Rudiger S, Schneider-Mergener J, and Bukau B (2001) Its substrate specificity characterizes the DnaJ co-chaperone as a scanning factor for the DnaK chaperone. EMBO J, 20, 1042-1050.

136. Rutkowski DT, Kang SW, Goodman AG, Garrison JL, Taunton J, Katze MG, Kaufman RJ, and Hegde RS (2007) The role of p58IPK in protecting the stressed endoplasmic reticulum. Mol Biol Cell, 18, 3681-3691.

137. Sadler I, Chiang A, Kurihara T, Rothblatt J, Way J, and Silver P (1989) A yeast gene important for protein assembly into the endoplasmic reticulum and the 
nucleus has homology to DnaJ, an Escherichia coli heat shock protein. $J$ Cell Biol, $109,2665-2675$.

138. Sahi C and Craig EA (2007) Network of general and specialty J protein chaperones of the yeast cytosol. Proc Natl Acad Sci U S A, 104, 7163-7168.

139. Saito H and Uchida H (1977) Initiation of the DNA replication of bacteriophage lambda in Escherichia coli K12. J Mol Biol, 113, 1-25.

140. Sandvig K, Garred O, Prydz K, Kozlov JV, Hansen SH, and van Deurs B (1992) Retrograde transport of endocytosed Shiga toxin to the endoplasmic reticulum. Nature, 358, 510-512.

141. Schlenstedt G, Harris S, Risse B, Lill R, and Silver PA (1995) A yeast DnaJ homologue, Scj1p, can function in the endoplasmic reticulum with BiP/Kar2p via a conserved domain that specifies interactions with Hsp70s. J Cell Biol, 129, 979988.

142. Schumacher RJ, Hansen WJ, Freeman BC, Alnemri E, Litwack G, and Toft DO (1996) Cooperative action of Hsp70, Hsp90, and DnaJ proteins in protein renaturation. Biochem, 35, 14889-14898.

143. Senderek J, Krieger M, Stendel C, Bergmann C, Moser M, Breitbach-Faller N, Rudnik-Schoneborn S, Blaschek A, Wolf NI, Harting I, North K, Smith J, Muntoni F, Brockington M, Quijano-Roy S, Renault F, Herrmann R, Hendershot LM, Schroder JM, Lochmuller H, Topaloglu H, Voit T, Weis J, Ebinger F, and Zerres K (2005) Mutations in SIL1 cause Marinesco-Sjogren syndrome, a cerebellar ataxia with cataract and myopathy. Nat Genet, 37, 1312-1314.

144. Sha B, Lee S, and Cyr DM (2000) The crystal structure of the peptide-binding fragment from the yeast Hsp40 protein Sis1. Structure, 8, 799-807.

145. Shen J, Chen X, Hendershot L, and Prywes R (2002) ER stress regulation of ATF6 localization by dissociation of BiP/GRP78 binding and unmasking of Golgi localization signals. Dev Cell, 3, 99-111.

146. Shen Y and Hendershot LM (2005) ERdj3, a stress-inducible endoplasmic reticulum DnaJ homologue, serves as a cofactor for BiP's interactions with unfolded substrates. Mol Biol Cell, 16, 40-50.

147. Shen Y and Hendershot LM (2007) Identification of ERdj3 and OBF-1/BOB1/OCA-B as direct targets of XBP-1 during plasma cell differentiation. $J$ Immunol, 179, 2969-2978.

148. Shen Y, Meunier L, and Hendershot LM (2002) Identification and characterization of a novel endoplasmic reticulum (ER) DnaJ homologue, which stimulates ATPase activity of BiP in vitro and is induced by ER stress. J Biol Chem, 277, 15947-15956. 
149. Silberstein S, Schlenstedt G, Silver PA, and Gilmore R (1998) A role for the DnaJ homologue Scjlp in protein folding in the yeast endoplasmic reticulum. $J$ Cell Biol, 143, 921-933.

150. Simons JF, Ferro-Novick S, Rose MD, and Helenius A (1995) BiP/Kar2p serves as a molecular chaperone during carboxypeptidase $\mathrm{Y}$ folding in yeast. J Cell Biol, $130,41-49$.

151. Skowronek MH, Hendershot LM, and Haas IG (1998) The variable domain of nonassembled Ig light chains determines both their half-life and binding to the chaperone BiP. Proc Natl Acad Sci U S A, 95, 1574-1578.

152. Skowronek MH, Rotter M, and Haas IG (1999) Molecular characterization of a novel mammalian DnaJ-like Sec63p homolog. Biol Chem, 380, 1133-1138.

153. Sondermann H, Scheufler C, Schneider C, Hohfeld J, Hartl FU, and Moarefi I (2001) Structure of a Bag/Hsc70 complex: convergent functional evolution of Hsp70 nucleotide exchange factors. Science, 291, 1553-1557.

154. Stuart JK, Myszka DG, Joss L, Mitchell RS, McDonald SM, Xie Z, Takayama S, Reed JC, and Ely KR (1998) Characterization of interactions between the antiapoptotic protein BAG-1 and Hsc70 molecular chaperones. J Biol Chem, 273, 22506-22514.

155. Suh WC, Burkholder WF, Lu CZ, Zhao X, Gottesman ME, and Gross CA (1998) Interaction of the Hsp70 molecular chaperone, DnaK, with its cochaperone DnaJ. Proc Natl Acad Sci U S A, 95, 15223-15228.

156. Szabo A, Korszun R, Hartl FU, and Flanagan J (1996) A zinc finger-like domain of the molecular chaperone DnaJ is involved in binding to denatured protein substrates. EMBO J, 15, 408-417.

157. Szabo A, Langer T, Schroder H, Flanagan J, Bukau B, and Hartl FU (1994) The ATP hydrolysis-dependent reaction cycle of the Escherichia coli Hsp70 system DnaK, DnaJ, and GrpE. Proc Natl Acad Sci U S A, 91, 10345-10349.

158. Tabibzadeh S and Broome J (1999) Heat shock proteins in human endometrium throughout the menstrual cycle. Infect Dis Obstet Gynecol, 7, 5-9.

159. Tang W and Wang CC (2001) Zinc fingers and thiol-disulfide oxidoreductase activities of chaperone DnaJ. Biochemistry, 40 (49) 14985-14994

160. Travers KJ, Patil CK, Wodicka L, Lockhart DJ, Weissman JS, and Walter P (2000) Functional and genomic analyses reveal an essential coordination between the unfolded protein response and ER-associated degradation. Cell, 101, 249-258.

161. Tsai B, Ye Y, and Rapoport TA (2002) Retro-translocation of proteins from the endoplasmic reticulum into the cytosol. Nat Rev Mol Cell Biol, 3, 246-255. 
162. Tsai J and Douglas MG (1996) A conserved HPD sequence of the J-domain is necessary for YDJ1 stimulation of Hsp70 ATPase activity at a site distinct from substrate binding. J Biol Chem, 271, 9347-9354.

163. Tyedmers J, Lerner M, Bies C, Dudek J, Skowronek MH, Haas IG, Heim N, Nastainczyk W, Volkmer J, and Zimmermann R (2000) Homologs of the yeast Sec complex subunits Sec62p and Sec63p are abundant proteins in dog pancreas microsomes. Proc Natl Acad Sci U S A, 97, 7214-7219.

164. Tyson JR and Stirling CJ (2000) LHS1 and SIL1 provide a lumenal function that is essential for protein translocation into the endoplasmic reticulum. $E M B O J, 19$, 6440-6452.

165. Vanhove M, Usherwood YK, and Hendershot LM (2001) Unassembled Ig heavy chains do not cycle from $\mathrm{BiP}$ in vivo but require light chains to trigger their release. Immunity, 15, 105-114.

166. Vogel M, Bukau B, and Mayer MP (2006a) Allosteric regulation of Hsp70 chaperones by a proline switch. Mol Cell, 21, 359-367.

167. Vogel M, Mayer MP, and Bukau B (2006b) Allosteric regulation of Hsp70 chaperones involves a conserved interdomain linker. J Biol Chem, 281, 3870538711.

168. Wall D, Zylicz M, and Georgopoulos C (1994) The NH2-terminal 108 amino acids of the Escherichia coli DnaJ protein stimulate the ATPase activity of DnaK and are sufficient for lambda replication. $J$ Biol Chem, 269, 5446-5451.

169. Wall D, Zylicz M, and Georgopoulos C (1995) The conserved G/F motif of the DnaJ chaperone is necessary for the activation of the substrate binding properties of the DnaK chaperone. J Biol Chem, 270, 2139-2144.

170. Walsh P, Bursac D, Law YC, Cyr D, and Lithgow T (2004) The J-protein family: modulating protein assembly, disassembly and translocation. EMBO Rep, 5, 567571.

171. Walter P and Blobel G (1983) Preparation of microsomal membranes for cotranslational protein translocation. Methods Enzymol, 96:84-93, 84-93.

172. Wawrzynow A, Banecki B, Wall D, Liberek K, Georgopoulos C, and Zylicz M (1995) ATP hydrolysis is required for the DnaJ-dependent activation of DnaK chaperone for binding to both native and denatured protein substrates. Journal of Biological Chemistry, 270, 19307-19311.

173. Wawrzynow A and Zylicz M (1995) Divergent effects of ATP on the binding of the DnaK and DnaJ chaperones to each other, or to their various native and denatured protein substrates. Journal of Biological Chemistry, 270, 19300-19306. 
174. Wei J and Hendershot LM (1996) Protein folding and assembly in the endoplasmic reticulum. EXS, 77, 41-55.

175. Wei J-Y, Gaut JR, and Hendershot LM (1995) In vitro dissociation of BiP:peptide complexes requires a conformational change in BiP after ATP binding but does not require ATP hydrolysis. $J$ Biol Chem, 270, 26677-26682.

176. Werner ED, Brodsky JL, and McCracken AA (1996) Proteasome-dependent endoplasmic reticulum-associated protein degradation: an unconventional route to a familiar fate. Proc Natl Acad Sci U S A, 93, 13797-13801.

177. Westermann B, Prip-Buus C, Neupert W, and Schwarz E (1995) The role of the GrpE homologue, Mge1p, in mediating protein import and protein folding in mitochondria. EMBO J, 14, 3452-3460.

178. Whitley P, Nilsson IM, and von Heijne G (1996) A nascent secretory protein may traverse the ribosome/endoplasmic reticulum translocase complex as an extended chain. J Biol Chem, 271, 6241-6244.

179. Wittung-Stafshede P, Guidry J, Horne BE, and Landry SJ (2003) The J-domain of Hsp40 couples ATP hydrolysis to substrate capture in Hsp70. Biochem, 42, 49374944.

180. Wu Y, Li J, Jin Z, Fu Z, and Sha B (2005) The crystal structure of the C-terminal fragment of yeast Hsp40 Ydj1 reveals novel dimerization motif for Hsp40. J Mol Biol, 346, 1005-1011

181. Yochem J, Uchida H, Sunshine M, Saito H, Georgopoulos CP, and Feiss M (1978) Genetic analysis of two genes, dnaJ and dnaK, necessary for Escherichia coli and bacteriophage lambda DNA replication. Mol Gen Genet, 164, 9-14.

182. Yoshida H, Haze K, Yanagi H, Yura T, and Mori K (1998) Identification of the cis-acting endoplasmic reticulum stress response element responsible for transcriptional induction of mammalian glucose-regulated proteins. Involvement of basic leucine zipper transcription factors. J Biol Chem, 273, 33741-33749.

183. Yoshida H, Okada T, Haze K, Yanagi H, Yura T, Negishi M, and Mori K (2000) ATF6 activated by proteolysis binds in the presence of NF-Y (CBF) directly to the cis-acting element responsible for the mammalian unfolded protein response. Mol Cell Biol, 20, 6755-6767.

184. Youker RT, Walsh P, Beilharz T, Lithgow T, and Brodsky JL (2004) Distinct roles for the Hsp40 and Hsp90 molecular chaperones during cystic fibrosis transmembrane conductance regulator degradation in yeast. Mol Biol Cell, 15, $4787-4797$ 
185. Yu M, Haslam RH, and Haslam DB (2000) HEDJ, an Hsp40 co-chaperone localized to the endoplasmic reticulum of human cells. J Biol Chem, 275, 2498424992.

186. Zhang Y, Nijbroek G, Sullivan ML, McCracken AA, Watkins SC, Michaelis S, and Brodsky JL (2001) Hsp70 molecular chaperone facilitates endoplasmic reticulum-associated protein degradation of cystic fibrosis transmembrane conductance regulator in yeast. Mol Biol Cell, 12, 1303-1314.

187. Zhao L, Longo-Guess C, Harris BS, Lee JW, and Ackerman SL (2005) Protein accumulation and neurodegeneration in the woozy mutant mouse is caused by disruption of SIL1, a cochaperone of BiP. Nat Genet, 37, 974-979.

188. Zhu X, Zhao X, Burkholder WF, Gragerov A, Ogata CM, Gottesman ME, and Hendrickson WA (1996) Structural analysis of substrate binding by the molecular chaperone DnaK. Science, 272, 1606-1614. 
APPENDIX A. SUPPLEMENTAL MATERIAL FOR CHAPTER 3 


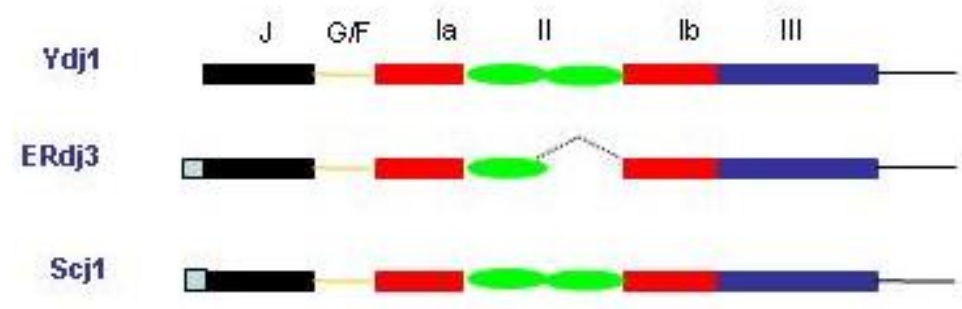

Figure A-1. Schematic drawings of the domain structure of Ydj1, Scj1 and ERdj3

According to Ydj1's crystal structure, domain II is intercepted in domain I and separates it into two halves: Ia and Ib, which fold together to form the intact substrate binding site. Scj1 is a type I DnaJ protein in yeast ER. It is predicted to have the similar structure as Ydj1 except the N-terminus ER signal sequence, which is cleaved from the mature protein. ERdj3 is considered to be a type I DnaJ protein also, although it contains a smaller domain II. light blue: ER signal sequence; black: J domain; yellow: G/F; red: domain I; green: domain II; blue: domain III. 


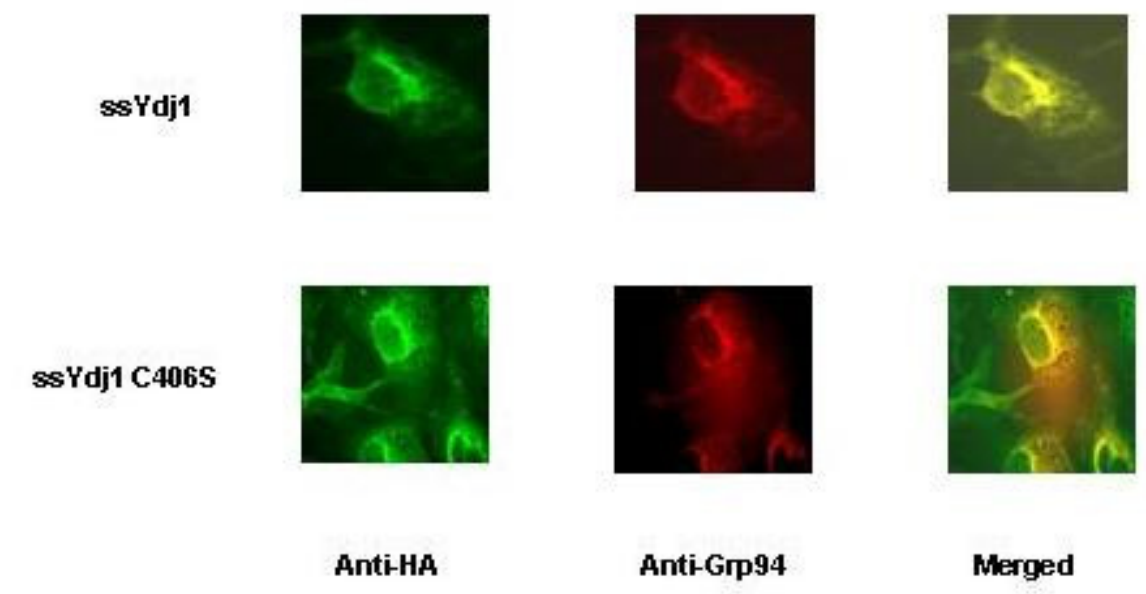

Figure A-2. Expression of Ydj1 in mammalian ER

Two ER targeting form of Ydj1 were expressed in COS-1 cells that had been grown on coverslips. ss Ydj1 has an ER signal sequence engineered at it $\mathrm{N}$-terminus, and ss $\mathrm{Ydj} 1$ $\mathrm{C} 406 \mathrm{~S}$ was generated by adding the $\mathrm{N}$-terminus signal sequence and removing the $\mathrm{C}$ terminus farnesylation site. Both are constructed in the 3HA-DSL vector and have Cterminus HA-tag. Cells were fixed and stained with an anti-HA antibody followed by FITC goat-anti-mouse IgG. Grp94, an abundant ER luminal protein that served as control for ER localization, was detected with an anti-Grp94 antiserum followed by TRITC conjugated goat-anti-rabbit IgG. 


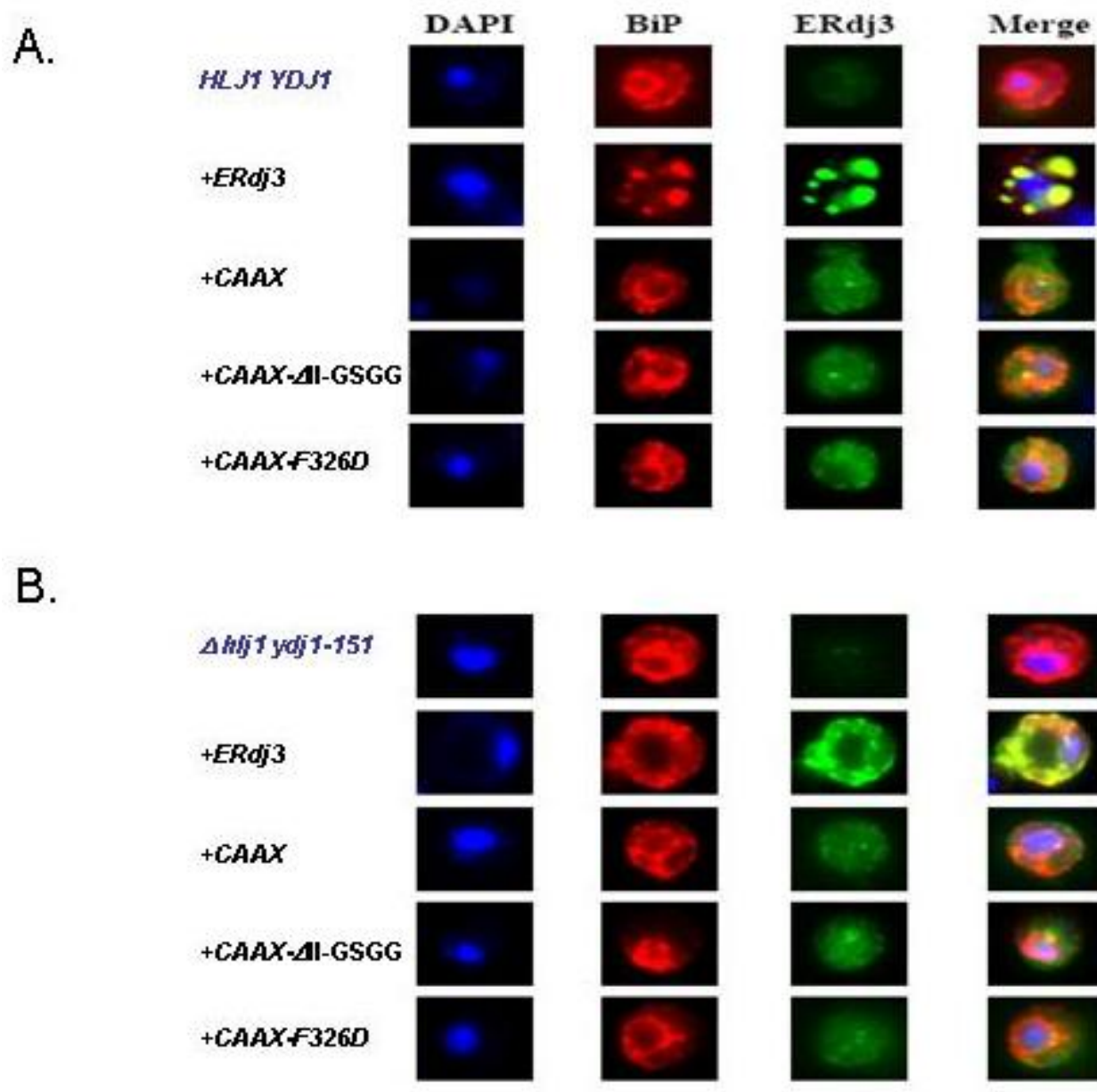

Figure A-3. Expression of the various wild-type and mutant ERdj3 in yeast

Wild-type HLJ1YDJl (A) and mutant hlj1 $1 y d j 1-151$ (B) yeast strains containing an empty vector (-), or expression vectors for an ER-targeted form of ERdj3 (ssERdj3), an ER-tethered cytosolically localized form of full-length ERdj3 (CaaX) or CaaX mutants (CaaX-AII-GSGG, CaaX-F326D, CaaX-I134A, CaaX-L208A and CaaX-IVLFa) were immunostained with antibodies against ER-lumenal Kar2p (TRITC labeled secondary antibody) and ERdj3 (FITC labeled secondary antibody). DAPI was used to visualize the nuclear DNA. 
APPENDIX B. SUPPLEMENTAL MATERIAL FOR CHAPTER 4 


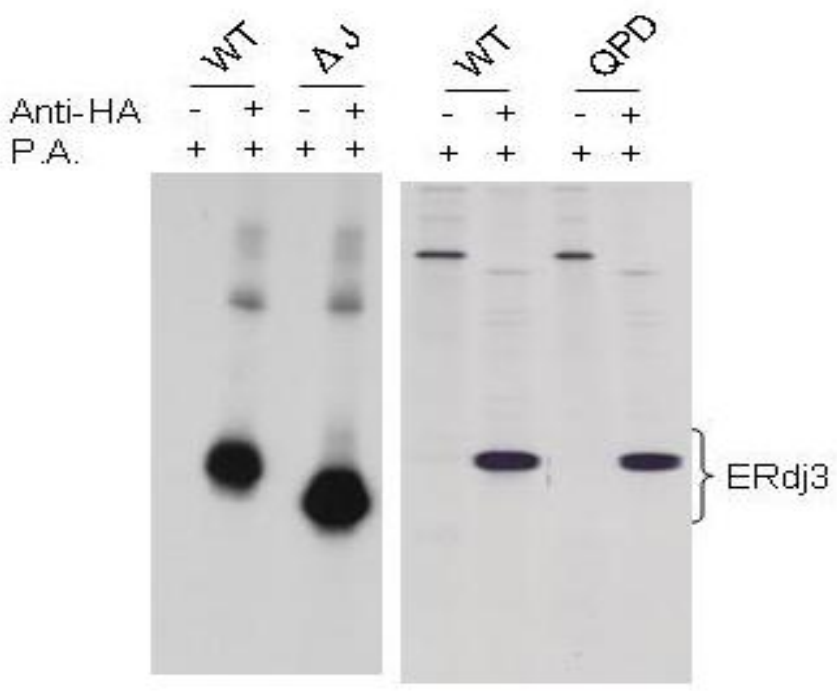

Figure B-1. WT/ $\triangle \mathrm{J}$ ERdj3 do not bind to Protein A Sepharose beads by themselves

Cos- 1 cells were transfected with cDNAs encoding HA-tagged versions of wild type ERdj3, a mutant that lacked the J domain $(\Delta \mathrm{J})$ or one that had a mutation which disrupted the $\mathrm{BiP}$ interaction site $(\mathrm{HPD} \rightarrow \mathrm{QPD})$. Forty hours post-transfection, cells were metabolically labeled for 3 hrs and treated with DSP as in Figure 4-1. After quenching, cell lysates were made and immunoprecipitated with either anti-HA or Protein A Sepharose alone. Isolated proteins were separated by reducing SDS-PAGE. 


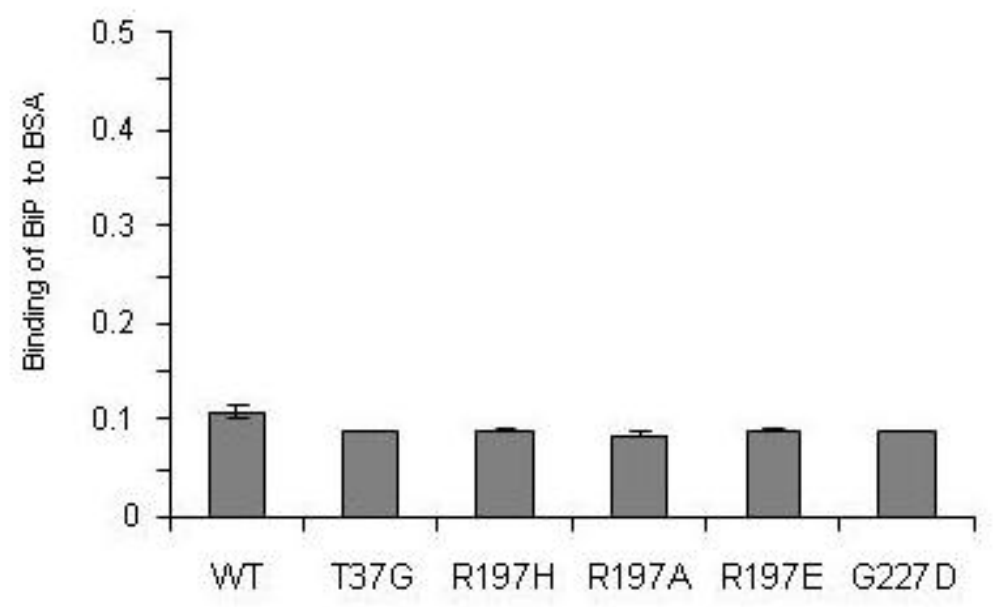

Figure B-2. Neither WT nor mutants BiP bind non-specifically to BSA blocked wells

Plate ELISA assays were performed as in Figure 4-4 except that denatured luciferase was not used to coat the wells. Briefly, $200 \mu \mathrm{l}$ of PBS containing $1 \%$ BSA was added to block the wells and incubate for $1 \mathrm{hr}$ at RT. After washing, recombinant wild-type or the indicated BiP mutant proteins $(5 \mu \mathrm{M})$ were added and incubated for $1 \mathrm{hr}$ at RT. After washing, the amount of BiP that bound non-specifically to the wells was detected with a polyclonal anti-BiP antiserum, followed by donkey anti-rabbit Ig conjugated to alkaline phosphatase. The signal was detected as described in Figure 4-4. 


\section{VITA}

Yi Jin was born in Shanghai, China in 1974. She attended Wuhan University in Wuhan, China, in 1992, where she received her Bachelor of Science degree in Biochemistry in July of 1996. After that, she worked in Wuhan Institution of Biological Products under the Ministry of Public Health. She subsequently entered the graduate program in the Department of Biology at the East China Normal University (ECNU) in Shanghai, China in 1999. After graduating from ECNU in 2002, she participated in the PhD program of Department of Molecular Sciences, at the University of Tennessee, Memphis. In the Spring of 2003, she joined the laboratory of Dr. Linda Hendershot. 